Engineering Models of

Deflagration-to-Detonation Transition

John B. Bdzil

Steven F. Son 



\section{DISCLAIMER}

This report was prepared as an account of work sponsored by an agency of the United States Government. Neither the United States Government nor any agency thereof, nor any of their employees, make any warranty, express or implied, or assumes any legal liability or responsibility for the accuracy, completeness, or usefulness of any information, apparatus, product, or process disclosed, or represents that its use would not infringe privately owned rights. Reference herein to any specific commercial product, process, or service by trade name, trademark, manufacturer, or otherwise does not necessarily constitute or imply its endorsement, recommendation, or favoring by the United States Government or any agency thereof. The views and opinions of authors expressed herein do not necessarily state or reflect those of the United States Government or any agency thereof. 


\section{DISCLAIMER}

Portions of this document may be illegible in electronic image products. Images are produced from the best available original document. 


\section{Contents}

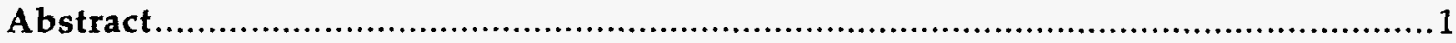

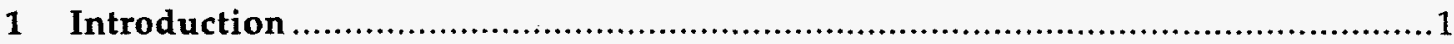

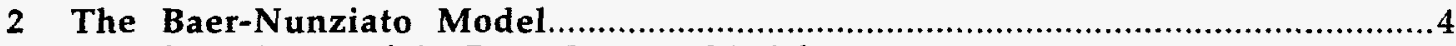

2.1 Description of the Baer-Nunziato Model ...................................................

2.2 Properties of the BN Model......................................................................

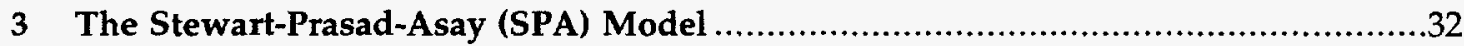

4 The Bdzil-Kapila-Stewart (BKS) Model.................................................................39

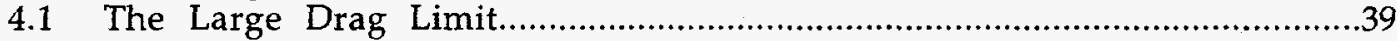

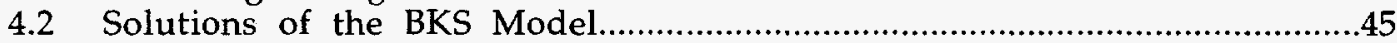

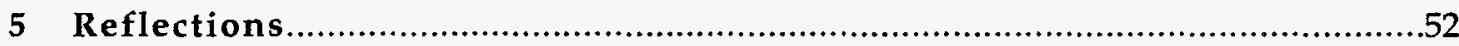

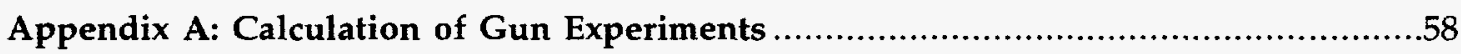

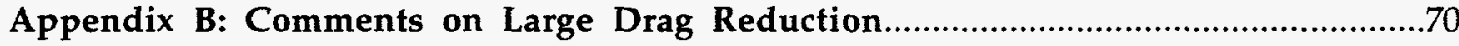




\title{
ENGINEERING MODELS \\ OF DEFLAGRATION-TO-DETONATION TRANSITION
}

by

John B. Bdzil and Steven F. Son

\begin{abstract}
For the past two years, Los Alamos has supported research into the deflagration-todetonation transition (DDT) in damaged energetic materials as part of the explosives safety program. This program supported both a theory/modeling group and an experimentation group. The goal of the theory/modeling group was to examine the various modeling structures (one-phase models, two-phase models, etc.) and select from these a structure suitable to model accidental initiation of detonation in damaged explosives. The experimental data on low-velocity piston supported DDT in granular explosive was to serve as a test bed to help in the selection process. Three theoretical models have been examined in the course of this study: (1) the Baer-Nunziato (BN) model, (2) the Stewart-Prasad-Asay (SPA) model and (3) the Bdzil-Kapila-Stewart model. Here we describe these models, discuss their properties, and compare their features.
\end{abstract}

\section{Introduction}

The goal of the explosives safety program is to understand how explosives respond to unconventional stimuli. In a review of the potential accident scenarios in which the explosives in a nuclear weapon undergo detonation, Larry Hantel concluded that the only credible scenarios involved weak shock and thermal insults rather than strong shocks [1]. These insults could lead to detonation via many different paths and could involve (1) damage of the explosives, (2) subsequent ignition of the damaged explosive by either a weak mechanical or thermal stimulus, leading to (3) burning of the explosive and ultimately to (4) detonation of the damaged explosives. The physics controlling these processes includes relatively slow, subsonic processes such as fracture, heat conduction, compaction and deflagration and fast, supersonic processes such as detonation. The relation between state variables such as pressure and temperature would typically be quite different in the slow and fast processes. For example, the pressure is nearly spatially uniform and the velocity small in subsonic flows, whereas the pressure and velocity more nearly follow a Hugoniot for supersonic flows. Thus a high-explosive safety model must have a true multiprocess capability.

For the model to be effective, it is crucial that the important state variables be identified and their evolution be cleanly separated and modeled. This leads one to adopt a different modeling philosophy than the one commonly used to formulate shock-initiation models. Since shock initiation of detonation is more nearly a single process phenomenon, successful models were built around a variety of equation of state assumptions. Any discrepancies in the description were then absorbed into the chemical-rate model. In fact, the 
common practice in shock-initiation modeling was to pick an equation of state model (such as temperature and pressure equilibrium between the solid and gas phases) based solely on subjective reasons and issues of convenience. Thus one of the first goals of the theory and modeling group was to select the basic structure for a deflagration-to-detonation transition (DDT) model.

The problem of accidental initiation of detonation in previously damaged explosives was the initial focus problem of the Los Alamos DDT program. As an absolute minimum, the modeling of this problem requires the inclusion of a damage variable in addition to the standard variable set; the conventional explosives modeling methodology considers mass, momentum, energy and degree of reaction variables (4 variables) [2]. In the standard approach, every initial physical state of the explosive (e.g., \% of theoretical maximum density (TMD)) requires an independent experimental calibration of the evolution equation (rate law) governing the degree of reaction variable. Since the state of damage (say as measured by the porosity) is dependent on the nature of the insult that the explosive sustains, the standard four variable approach was hardly adequate. Thus the initial goal of the DDT modeling group was to develop a model containing a porosity variable that could explain the phenomonology of the DDT-tube test results for granular HMX explosives. This was selected as the focus problem for two reasons; (1) the porosity and changes in porosity have a strong but poorly understood influence on the observed DDT of this material and (2) a high-quality data base exists to test models against.

A considerable amount of work was done on the DDT problem during the $1980 \mathrm{~s}$. In the area of theory and modeling, the pace-setting work of Baer and Nunziato at Sandia Laboratories most effectively married sound theory with experimental data to produce an engineering tool [3]. Since "reinventing the wheel" serves no one's best interests, we decided to carefully examine the multiphase Baer-Nunziato (BN) model as one of our candidate models. The second candidate model is the Stewart-Prasad-Asay (SPA) model. This model adds a porosity variable to the standard detonation modeling methodology described above. The third model, developed in the course of this study, is the Bdzil-Kapila-Stewart (BKS) model. The goal was to test the assumptions made by these models both by looking at the solutions to the models themselves and by comparing their "predictions" against experiment.

What we do in this report is start by describing the porosity-variable-containing $\mathrm{BN}$ model, and show how the SPA and BKS models are obtained as special limits of BN. The object is to study the most complex existing engineering model of DDT (the 7-equation $\mathrm{BN}$ model) and (1) seek reduced, simpler models that retain the same level of "predictive" capability and then (2) improve on the overall physics in the models. This report is mostly of a historical nature; we describe the developments we have made in DDT modeling over the past 2 years.

All the models we consider are continuum mechanics models. They conserve overall mass, momentum, and energy. In addition, the degree of reaction and porosity are described through partial differential equations (PDEs) and the material response is described with a combination of algebraic and differential constitutive relations. The structural differences in these models are a consequence of differences in the material response functions. All three models consider that the material can be in one of two phases; (1) unreacted (solid) and (2) reacted (gas). The differences in the models is largely a result of the closure laws 
that are used to describe the interaction between these phases. The three models have the following structure:

- the 7-equation BN model

1. independent conservation equations for mass, momentum, and energy for each phase (for a total of 6-equations)

2. porosity evolution equation describes both compaction and reaction (1-equation),

- the 5-equation SPA model

1. overall conservation equations for mass, momentum, and energy (for a total of 3-equations)

2. porosity evolution equation for compaction and reaction progress equation (2equations), and

- the 6-equation BKS model

1. in the physically relevant limit of large interphase drag, the BN model reduces from two equations for momentum conservation to one equation (6-equations).

A summary of our methods and results is given below.

Executive Summary

- This progress report describes the work of the theory/modeling group over the past 2 years.

- Effort has been directed toward studying the underlying physical assumptions and mathematical structure of DDT models.

- The work of Baer and Nunziato has been reviewed and some misconceptions corrected.

- Two new models have evolved, and their properties have been studied.

- Results obtained with the three models have been carefully compared. The BN model disagrees with the other for reasons that are not totally understood.

- One of the new models, a 7-equation BKS model, seems the best choice.

We will now describe each of these models, including information on their development, the properties of their solutions, and their ability to describe the experiments. The BN model is considered first. 


\section{The Baer-Nunziato Model}

\subsection{Description of the Baer-Nunziato Model}

Since the BN was introduced 9 years ago it has undergone many changes. These changes have largely been made to the "chemistry" part of the model. Many of these more recent changes are only weakly supported by the experimental data base. Therefore, we have chosen one of its most basic forms and called that form the BN model. In order to be included in this model, the interactions and supporting parameters had to pass a test; they had to have independent experimental support and verification. This unadorned version of the BN model is

$$
\begin{aligned}
& \text { solid mass } \quad\left(\phi_{s} \rho_{s}\right)_{t}+\left(\phi_{s} \rho_{s} V_{s}\right)_{x}=C_{s}^{\dagger}, \\
& \text { gas mass } \\
& \left(\phi_{g} \rho_{g}\right)_{t}+\left(\phi_{g} \rho_{g} V_{g}\right)_{x}=-C_{s}^{\dagger} \\
& \underline{\text { solid momentum }}\left(\phi_{s} \rho_{s} V_{s}\right)_{t}+\left(\phi_{s} \rho_{s} V_{s}^{2}+\phi_{s} P_{s}\right)_{x}=C_{s}^{\dagger} V_{s} \\
& +P_{g}\left(\phi_{s}\right)_{x}-\left(\delta+C_{s}^{\dagger} / 2\right)\left(V_{s}-V_{g}\right) \\
& \text { gas momentum } \\
& \left(\phi_{g} \rho_{g} V_{g}\right)_{t}+\left(\phi_{g} \rho_{g} V_{g}^{2}+\phi_{g} P_{g}\right)_{x}=-C_{s}^{\dagger} V_{s} \\
& -P_{g}\left(\phi_{s}\right)_{x}+\left(\delta+C_{s}^{\dagger} / 2\right)\left(V_{s}-V_{g}\right) \\
& \underline{\text { solid energy }} \\
& {\left[\phi_{s} \rho_{s}\left(e_{s}+V_{s}^{2} / 2\right)\right]_{t}+\left[\phi_{s} \rho_{s}\left(e_{s}+V_{s}^{2} / 2\right) V_{s}+\phi_{s} P_{s} V_{s}\right]_{x}=\mathcal{E}} \\
& +\left(e_{s}+V_{s}^{2} / 2\right) C_{s}^{\dagger}+\left[P_{g}\left(\phi_{s}\right)_{x}-\left(\delta+C_{s}^{\dagger} / 2\right)\left(V_{s}-V_{g}\right)\right] V_{s}, \\
& \text { gas energy } \\
& {\left[\phi_{g} \rho_{g}\left(e_{g}+V_{g}^{2} / 2\right)\right]_{t}+\left[\phi_{g} \rho_{g}\left(e_{g}+V_{g}^{2} / 2\right) V_{g}+\phi_{g} P_{g} V_{g}\right]_{x}=-\mathcal{E}} \\
& -\left(e_{s}+V_{s}^{2} / 2\right) C_{s}^{\dagger}-\left[P_{g}\left(\phi_{s}\right)_{x}-\left(\delta+C_{s}^{\dagger} / 2\right)\left(V_{s}-V_{g}\right)\right] V_{s},
\end{aligned}
$$

with

$$
\mathcal{E}=-\left\{\left(P_{s}-\beta_{s}\left(\phi_{s}\right)\right) \mathcal{F}+\mathcal{H}\left(T_{s}-T_{g}\right)\right\} \quad \text { or } \quad \mathcal{E}=-\left\{P_{g} \mathcal{F}+\mathcal{H}\left(T_{s}-T_{g}\right)\right\},
$$


and where the compaction law is

$$
\mathcal{F}=\frac{\phi_{s}\left(1-\phi_{s}\right)}{\mu_{c}}\left(P_{s}-\beta_{s}\left(\phi_{s}\right)-P_{g}\right)
$$

the major energy-releasing reaction obeys

$$
C_{s}^{\dagger}=-3 \star e t i \star\left(\phi_{s} \rho_{s}\right) \star a c o e f \star\left(P_{g} / A_{p s}\right)^{b n} \star(1-f d v)^{\frac{2}{3}} \star(1 / a),
$$

and the saturation condition is

$$
\phi_{s}+\phi_{g}=1
$$

We will use subscript notation where it is unambiguous to denote partial derivatives with respect to $t$ and $x$. The following list of symbols defines the variables appearing in these equations. The subscript $i$ takes on two values; $i=s$ corresponds to solid and $i=g$ corresponds to gas:

$$
\begin{aligned}
& \text { Symbols (MKS-units) } \\
& \begin{array}{ll}
\text { - } & \phi_{s} \\
& \rho_{i}\left(\mathrm{~kg} / \mathrm{m}^{3}\right)
\end{array} \\
& \text { - } V_{i}(\mathrm{~m} / \mathrm{s}) \\
& \text { - } P_{i}\left(\mathrm{~N} / \mathrm{m}^{2}\right) \\
& \text { - } \quad e_{i}(\mathrm{~N} \cdot \mathrm{m} / \mathrm{kg}) \\
& \text { - } T_{i}(\mathrm{~K}) \\
& \text { - } C_{s}^{\dagger}\left(\mathrm{kg} / \mathrm{m}^{3} / \mathrm{s}\right) \\
& =0 \\
& =1 \\
& =0.1227 \mathrm{~m} / \mathrm{s} \\
& =0.1139 \mathrm{~m} / \mathrm{s} \\
& =1.0 \\
& =0.8 \\
& (1-f d v)^{\frac{2}{3}} \\
& =10^{8} \mathrm{~N} / \mathrm{m}^{2} \\
& f d v=\left(\phi_{s}-\phi_{s}{ }^{o}\right) /\left(1-\phi_{s}{ }^{o}\right) \\
& f d v=0.0
\end{aligned}
$$


$\underline{\text { Symbols (MKS-units) }}$

$a$

$$
\begin{gathered}
=a p \\
=a p\left(\phi_{s}{ }^{o} / \phi_{s}\right)^{\frac{1}{3}}
\end{gathered}
$$

$a p$

$$
=7.5 \times 10^{-5} \mathrm{~m}
$$

- $\delta\left(\mathrm{kg} / \mathrm{m}^{3} / \mathrm{s}\right)$

$$
\delta=\mu_{g}\left(1+\kappa_{2} \operatorname{Re} / \phi_{s}\right) \kappa_{1} /(2 a)^{2} / \phi_{g}{ }^{4.5}
$$

$$
\begin{aligned}
& \mu_{g} \\
& =8.51 \times 10^{-5} \mathrm{~kg} / \mathrm{m} / \mathrm{s}
\end{aligned}
$$

Re

$\kappa_{1}, \kappa_{2}$

$$
\kappa_{1}=150.0, \quad \kappa_{2}=0.01
$$

- $\beta_{s}\left(\mathrm{~N} / \mathrm{m}^{2}\right)$

$$
\begin{aligned}
\beta_{s}\left(\phi_{s}\right)=-\tau \star\left(\phi_{s}-\phi_{s}{ }^{o}\right) & \ln \left(\phi_{g}\right) / \phi_{g} \\
& =0 \\
\tau & =1.27 \times 10^{7} \mathrm{~N} / \mathrm{m}^{2}
\end{aligned}
$$

- $\mathcal{F}(1 / \mathrm{s})$

$$
\begin{aligned}
\mathcal{F}=\left(\phi_{s} \phi_{g} / \mu_{c}\right)\left(P_{s}-\beta_{s}\left(\phi_{s}\right)\right. & \left.-P_{g}\right) \\
& =-\phi_{s} \phi_{g} P_{g} / \mu_{c}
\end{aligned}
$$

$$
=100 \mathrm{~kg} / \mathrm{m} / \mathrm{s}
$$

- $\quad \mathcal{H}\left(\mathrm{N} / \mathrm{m}^{2} / \mathrm{s} / \mathrm{K}\right) \star\left(T_{s}-T_{g}\right)$

$$
\begin{gathered}
\mathcal{H}=3 \star \phi_{s} \star k_{g} \star\left(1+0.4\left(R e^{2} P r\right)^{\frac{1}{3}}\right) / a^{2} \\
k_{g}=9.427 \times 10^{-2} \mathrm{~N} / \mathrm{s} / \mathrm{K} \\
\operatorname{Pr} \\
=0.75
\end{gathered}
$$

\section{Definitions}

solid particle radius

"factor"

$\phi_{s} \geq \phi_{s}{ }^{o}$

$\phi_{s}<\phi_{s}{ }^{o}$

initial solid particle

"mean" radius

interphase drag coefficient (phase momentum exchange)

$\underline{\text { gas viscosity }}$

Reynold's number

porous bed

permeability coefficients

intergranular stress

$\frac{P-\alpha \text { law }}{\phi_{s}>\phi_{s}^{o}}$

$\phi_{s} \leq \phi_{s}^{o}$

stress coefficient

compaction rate law

$P_{s}>\beta_{s}\left(\phi_{s}\right)$

$P_{s} \leq \beta_{s}\left(\phi_{s}\right)$

compaction viscosity

interphase heat transfer Newton's law coefficient

$\underline{\text { gas thermal conductivity }}$

gas Prandtl number

The particular forms that are used for the burn rate $C_{s}^{\dagger}$, the drag coefficient $\delta$, the intergranular stress $\beta_{s}\left(\phi_{s}\right)$, the compaction rate law $\mathcal{F}$, the heat transfer coefficient $\mathcal{H}$ and their 
related constants are taken from Baer's one-dimensional (1D), method-of-lines (MOL) code. The original BN model used $\left(\phi_{s} T_{s}+\phi_{g} T_{g}>T_{i g n}\right)$ to switch eti. Here we have adopted a pressure criterion. These expressions are intended to provide a leading order description of granular HMX. Equations of state (EOS) for the thermal (i.e., $T_{i}$ ) and mechanical (i.e., $P_{i}$ ) response of both the solid and gas also need to be provided

$$
T_{i}\left(e_{i}, \rho_{i}\right)
$$

and

$$
P_{i}\left(T_{i}, \rho_{i}\right)
$$

BN uses the Hayes Helmholtz free energy EOS for the solid (see Sheffield, Gustavsen and Alcon [4])

$$
\begin{aligned}
F_{s}\left(T_{s}, \rho_{s}\right) & =P_{s}^{o}\left(1-\rho_{s}^{o} / \rho_{s}\right) / \rho_{s}^{o}+C_{v s}\left\{\left(T_{s}-T_{s}^{o}\right) \star\left(1+g o v \star\left(1-\rho_{s}^{o} / \rho_{s}\right) / \rho_{s}^{o}\right)\right. \\
& \left.+T_{s} \ln \left(T_{s}^{o} / T_{s}\right)\right\}+t 4 \star\left\{\left(\rho_{s} / \rho_{s}{ }^{o}\right)^{n-1}-(n-1) \star\left(1-\rho_{s} / \rho_{s}^{o}\right)-1\right\}
\end{aligned}
$$

and derives

thermal

$$
\begin{aligned}
& T_{s}\left(e_{s}, \rho_{s}\right)=T_{s}^{o}+\frac{1}{C_{v s}}\left\{e_{s}+\left(t 3-P_{s}^{o} / \rho_{s}^{o}\right) \star\left(1-\rho_{s}^{o} / \rho_{s}\right)\right. \\
& \left.-t 4 \star\left[\left(\rho_{s} / \rho_{s}^{o}\right)^{n-1}-(n-1) \star\left(1-\rho_{s}^{o} / \rho_{s}\right)-1\right]\right\},
\end{aligned}
$$

mechanical $P_{s}\left(T_{s}, \rho_{s}\right)=P_{s}^{o}+C_{v s} \star g o v \star\left(T_{s}-T_{s}{ }^{o}\right)$

$$
+a k t o \star\left[\left(\rho_{s} / \rho_{s}^{o}\right)^{n}-1\right] / n
$$

and a JWL EOS for the gas

$$
\begin{array}{ll}
\text { thermal } & T_{g}\left(e_{g}, \rho_{g}\right)=T_{g}{ }^{o}+\frac{1}{C_{v_{g}}}\left\{e_{g}+h r e l\right. \\
& -\frac{1}{d m 1}\left[\left(A / R_{1}\right) \exp \left(-R_{1} \star d m 1 / \rho_{g}\right)\right. \\
& \left.\left.+\left(B / R_{2}\right) \exp \left(-R_{2} \star d m 1 / \rho_{g}\right)\right]\right\}, \\
\text { mechanical } \quad & P_{g}\left(T_{g}, \rho_{g}\right)=A \exp \left(-R_{1} \star d m 1 / \rho_{g}\right)+B \exp \left(-R_{2} \star d m 1 / \rho_{g}\right) \\
& +T_{g} \omega C_{v g} \rho_{g} .
\end{array}
$$

The parameters appearing in the above EOSs and the values used for HMX are given below: 
Symbols (MKS-units)

- $C_{v i}(\mathrm{~N} \cdot \mathrm{m} / \mathrm{kg} / \mathrm{K})$

$$
C_{v s}=1.5 \times 10^{3}
$$

$$
C_{v g}=\left[2.4-0.28 \star\left(d m 1 \times 10^{-3}-1.3\right)\right] \times 10^{3}
$$

- $g \circ v\left(\mathrm{~kg} / \mathrm{m}^{3}\right)$

- $\quad \operatorname{akto}\left(\mathrm{N} / \mathrm{m}^{2}\right)$

$$
g o v=2.1 \times 10^{3}
$$

$$
\text { akto }=1.35 \times 10^{10}
$$

- $\mathrm{n}$

$$
\mathrm{n}=9.8
$$

- $\quad \mathrm{t} 3(\mathrm{~N} \cdot \mathrm{m} / \mathrm{kg})$

$$
\mathrm{t} 3=C_{v s} \star T_{s}{ }^{o} \star g o v / \rho_{s}{ }^{o}
$$

- $\quad \mathrm{t} 4(\mathrm{~N} \cdot \mathrm{m} / \mathrm{kg})$

$$
\mathrm{t} 4=a k t o / \rho_{s}^{o} / n /(n-1)
$$

- $\quad \mathrm{dm} 1\left(\mathrm{~kg} / \mathrm{m}^{3}\right)$

$$
\mathrm{dm} 1=\phi_{s}^{o} \rho_{s}^{o}
$$

Note: For pure gas problems $\left(\phi_{s}{ }^{\circ}=0\right), \mathrm{dm} 1$ must be selected such that the JWL calibration is valid in the density range of interest.

- $\quad h r e l(N \cdot m / k g)$

$$
\begin{aligned}
\text { hrel }=\left[7.91-4.33 \star\left(\mathrm{dm} 1 \times 10^{-3}-1.3\right)^{2}\right. \\
\left.-0.934 \star\left(\mathrm{dm} 1 \times 10^{-3}-1.3\right)\right] \times 10^{6}
\end{aligned}
$$

- $\quad \mathrm{A}\left(\mathrm{N} / \mathrm{m}^{2}\right)$

$$
\begin{aligned}
\mathrm{A}=\left[-8.005+21.39 \star\left(\mathrm{dm} 1 \times 10^{-3}\right)\right. & -16.23 \star\left(\mathrm{dm} 1 \times 10^{-3}\right)^{2} \\
+ & \left.4.595 \star\left(\mathrm{dm} 1 \times 10^{-3}\right)^{3}\right] \times 10^{11}
\end{aligned}
$$

- $\mathrm{B}\left(\mathrm{N} / \mathrm{m}^{2}\right)$

$$
\begin{aligned}
& \mathrm{B}=\left[0.014-0.0349 \star\left(\mathrm{dm} 1 \times 10^{-3}\right)\right.-0.0156 \star\left(\mathrm{dm} 1 \times 10^{-3}\right)^{2} \\
&\left.+0.026 \star\left(\mathrm{dm} 1 \times 10^{-3}\right)^{3}\right] \times 10^{11}
\end{aligned}
$$

\section{Definitions}

specific heat

$\rho_{s}{ }^{\circ} \star$ (Gruneisen gamma)

$1^{\text {st }}$ Hayes EOS parameter

$2^{\text {nd }}$ Hayes EOS parameter

solid loading density used in JWL calibration for granular HMX (default value) heat of detonation

$1^{\text {st }}$ JWL EOS parameter

$2^{\text {nd }}$ JWL EOS parameter 
$\frac{\text { Symbols (MKS-units) }}{R_{1}}$

$R_{1}=4.2$

- $\quad R_{2}$

$R_{2}=1.0$

$\omega=0.25$
Definitions

$3^{\text {rd }}$ JWL EOS parameter

$4^{\text {th }}$ JWL EOS parameter

$5^{\text {th }}$ JWL EOS parameter

As is clear from this rather lengthy description, even the unadorned BN model is quite complex. The version of BN used by Baer to model granular HMX adds another layer of complexity to the manner in which the ignition parameter eti is determined (see Appendix A). This was required in order that the general timing seen in the $(x, t)$ - diagram for Sandusky's DDT-tube test could be simulated.

The entropy inequality used by Baer and Nunziato to construct the interphase interaction terms does not give unique expressions for these terms [3]. Both forms for the energy exchange term given by Eqs. (8a, b) are compatible with the entropy inequality. We focus only on the component proportional to $\mathcal{F}$. Equation (8a) divides the work done during compaction between the solid and the gas, while Eq. (8b) deposits all the work into the solid. Physically, Eq. (8b) seems more reasonable since we don't anticipate that the gas will be heated much by the work we do crushing the solid. The original formulation of the BN model used Eq. (8a) [3], while more recent formulations drop this term altogether. This points out that not only is the model complex, but the form of the interaction terms is uncertain. Baer and Nunziato used the entropy inequality to select the form of the interaction terms. The role of entropy is somewhat overstated by BN. Perhaps a good analogy is that the entropy inequality is to the interaction terms what the driver's license examination is to driving a car. Although passing the examination is a requirement it certainly does not make one a good driver. Ultimately, the usefulness of the model is determined by the quality of the thinking that goes into formulating the phase interaction terms.

The BN model is a continuum mixture theory model. Specifically, each material is assumed to consist of several phases (two in the BN model) that coexist at each spatial location occupied by the material. At the highest-level of spatial resolution $\mathcal{L}_{2}$ considered by such a theory, the interfaces between the various phases are not considered explicitly. For example, if we consider a material consisting of solid particles embedded in a gas, the dimension of the solid particles $\mathcal{L}_{1}$ is taken to be small compared with the control element size $\mathcal{L}_{2}$. A schematic representation of the scales is shown in Figure 1. The interaction between the phases enters the theory via source terms that are added to the single phase conservation laws. In an "average" way, these terms describe the exchange of mass, momentum and energy that takes place across the solid/gas interface. These exchange terms are modeled as essentially bulk constitutive laws that, however, contain variables from both phases. These laws must be provided in addition to the standard single-phase EOS information. 


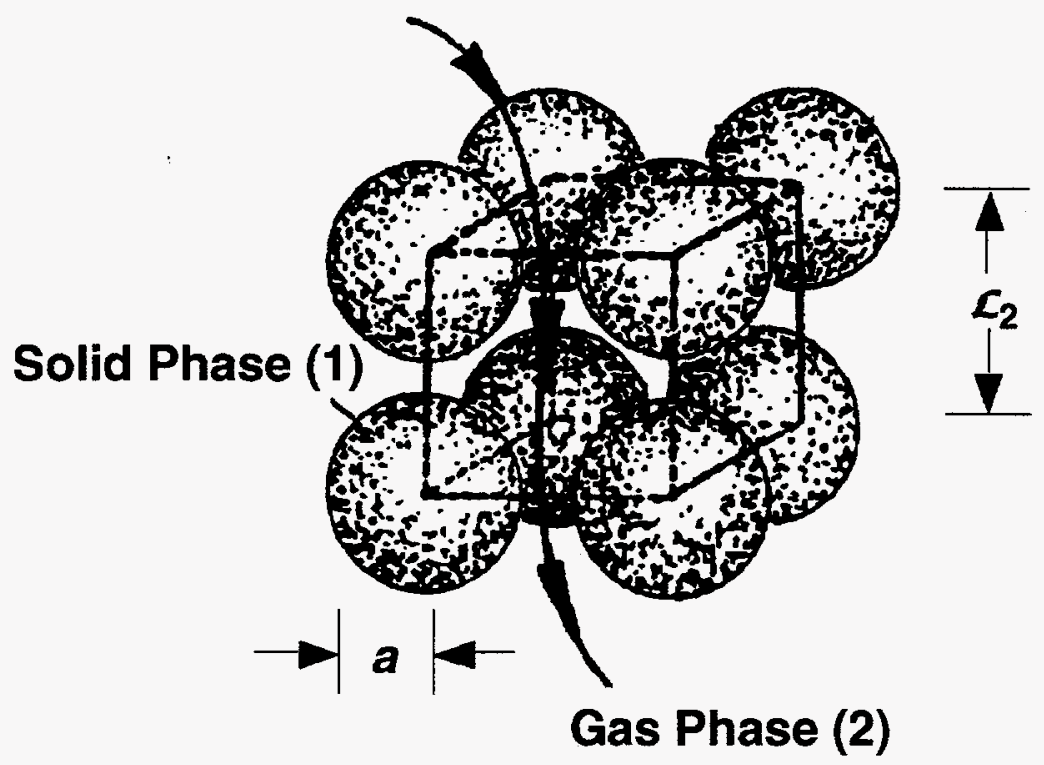

Fig. 1: $\mathcal{L}_{2}$ represents the smallest scale resolved in the continuum mixture approximation. The radius $a=\mathcal{L}_{1}$ of the solid particles (labeled as 1) satisfies $a<\mathcal{L}_{2}$. Phase 2 gas and phase 1 solid communicate with each other through their mutual interfaces (modified from [5]).

The conservation laws of the BN theory Eqs. (1)-(7) are of hyperbolic type. When these exchange terms are zero, the evolution of the phases is not coupled; each phase has its own evolving wave system. The exchange terms describe the interaction between the phases and as a consequence are symmetric between the phases. That is, mass or momentum that leaves one phase enters the other phase, such that the overall mass or momentum is conserved. The form taken by these exchange laws depends on the type of interaction being modeled.

Unique among these exchange terms are the "nozzling" terms

$$
P_{g}\left(\phi_{s}\right)_{x}
$$

and

$$
V_{s} P_{g}\left(\phi_{s}\right)_{x}
$$

they do not have adjustable parameters. Driven by a gradient in porosity, these terms mimic the effect on the gas flow that results from the effective change in the area of the virtual gas stream tube. Significantly, these terms are not in conservation form. Thus, the solutions to problems that have jumps in porosity, such as compaction waves when they are treated as discontinuities and material interfaces, are not uniquely defined by this model. Additional, microstructural information must be provided to properly capture the solution in the neighborhood of such features. Since the nozzling terms, Eqs. (23a, b), contain no parameters, these terms cannot be turned off; it is a permanent feature of the model. Not all two-phase, continuum mixture models contain this term. For example, the 
Powers-Stewart-Krier (PSK) model does not have this term [6]. The BKS model that we have derived has similar terms that are not in conservation form. In the BKS model these terms influence the shock structure, a common feature in all problems we do.

Typically, these laws are phenomenologically based and as such do not have universal validity. For example, a principal component of the momentum exchange is based on a laminar, slow flow, Stokes-like drag $\delta\left(V_{s}-V_{g}\right)$, where $\delta$ is the drag coefficient. This interaction controls both the momentum and energy exchange required to bring two mechanically dissimilar phases to velocity equilibrium and to maintain them in such a state. The Stokes drag form used here implies slow relative flows with $R e=2 a \star\left(\phi_{g} \rho_{g}\right)\left|V_{s}-V_{g}\right| / \mu_{g}<1$. For our applications, the drag is high and the flows are fast, which make the drag interaction $\left|\delta\left(V_{s}-V_{g}\right)\right|$ the fastest momentum and energy exchange process in the model. As we will show, large drag in fact leads to a simplification of the 2-velocity BN model to a 1-velocity model, the BKS model. This reduction brings about a simplification of the wave structure from a two-wave family theory to a one-wave family theory. Since $\delta$ does not appear in the BKS theory, deficiencies in its representation do not affect the BKS theory directly. However, the residue of the drag interaction is a new term in the theory that neither contains any adjustable parameters nor is in conservation form. This is reminiscent of the form and properties of the "nozzling" term discussed above.

Although the solid and gas are initially at the same temperature, most processes (such as shock passage, compaction and reaction) leave the solid and gas at different temperatures. As we have shown [7], the passage of a noncompacting shock over a mixture of solid and gas heats the solid more than the gas, and leaves a state of pressure and temperature disequilibrium. Subsequently, compaction deforms and further heats the solid and compresses the gas until a state of mechanical equilibrium is obtained. As the reactions begin, the solid is transformed into very hot gas, leading to the gas being considerably hotter than the solid. Thus most processes tend to prepare the mixture in a state of thermal disequilibrium. The heat transfer term, Eq. (15), acts to equalize these temperatures. It is a simple Newton's law of cooling, for which the exchange coefficient reflects the dominant role of the gas thermal conductivity and the inverse solid particle size; high conductivity and small particles enhance thermal equilibration. The trends displayed by Eq. (15) certainly seem plausible. The intent is that this expression be a model for convective heat transfer between an infinite feed of flowing gas and stationary solid [8]. Experiments show that this expression works well provided that $R e \neq 0$. When $R e=0$, the available experimental data are not consistent with this form [8]; the observed heat transfer is much slower than that predicted by Eq. (15).

As mentioned earlier, the passage of a compression wave over this two-phase solid/gas mixture tends to pressurize the solid in preference to the gas. This state of mechanical disequilibrium is resolved by Eq. (7) driven by the compaction law, Eq. (9). For the BN model, the state of mechanical equilibrium is given by

$$
P_{s}=\beta_{s}\left(\phi_{s}\right)+P_{g}
$$

where $\beta_{s}\left(\phi_{s}\right)$ is the configurational or intergranular stress, called the $P(\alpha)$ function in the Herrmann theory [9]. The function $\beta_{s}\left(\phi_{s}\right)$ is zero for the initial, porous, quiescent bed. It measures the level of stress required to produce a given compaction (deformation) of the porous bed. It can also be extended to predict the amount of damage when the solid is put 
into tension [10]. The porosity (damage) variable together with the intergranular stress, $\beta_{s}\left(\phi_{s}\right)$ are central variables in a model for damaged explosives. The assumed dependence of $\beta_{s}\left(\phi_{s}\right)$ on only $\phi_{s}$ excludes possible dependence of $\beta_{s}\left(\phi_{s}\right)$ on variables such as the specific internal energy $e_{s}$. In the treatment considered here, $\beta_{s}\left(\phi_{s}\right)$ describes a history independent process. Although data exist that show the state of compaction achieved is dependent on the rate of loading [4] [11], these effects are not considered here. We follow this simpler approach because the variations in $\beta_{s}\left(\phi_{s}\right)$ for granular HMX due to differences in particle morphology and the rate of stress loading are roughly comparable; a factor of $2-4$ increase in $\beta_{s}\left(\phi_{s}\right)$ for a given $\phi_{s}$ for dynamic relative to static loading. The quasi-static, isothermal data of Elban and Chiarito [12] for HMX are used to get $\beta_{s}\left(\phi_{s}\right)$. Nevertheless, Eq. (13) for $\beta_{s}\left(\phi_{s}\right)$ along with the compaction rate law, Eq. (14) used in Eq. (7), predicts the observed shortening of the rise time of pressure through a compaction wave with increasing piston speed [13]. These results are shown in Figure 2.

Two properties of the model lead to a steepening of the compaction wave profile with pressure. First, as the speed of the piston increases, the subsonic compaction wave becomes less dispersive the closer the speed of the wave gets to the sound speed in the granular material. For some piston speed, the compaction wave becomes supersonic. A noncompacting, hydrodynamic shock is then the first part of the compaction wave structure. The compaction process begins in the shocked material, leading to a further increase in the pressure. For a supersonic compaction wave, a significant increase in the pressure comes in a noncompacting shock. Second, the rate of compaction given by Eq. (14) is an increasing function of pressure. Thus at higher pressures, the compaction occurs more quickly.

Related to the compaction rate, $\mathcal{F}$ is an energy exchange law (source term) called, "compaction work"

$$
-\left(P_{s}-\beta_{s}\left(\phi_{s}\right)\right) \mathcal{F} \quad \text { or } \quad-P_{g} \mathcal{F} \text {. }
$$

The compaction work, which appears in the original but not the most recent version of the $\mathrm{BN}$ model, distributes the work done in compacting the solid between the solid and gas phases. This is described by Eq. (25a). Another possibility is that all the work done compacting the solid stays with the solid. This corresponds to Eq. (25b). Equation (25a) leads to extreme temperature increases in the gas, and was intended as a model for "hotspots." There is neither physical nor theoretical evidence for such a term. We included it to help us compare our work to that of Baer and Nunziato [3]. Physically, we expect the solid to be heated by the mechanical deformations described by $\beta_{s}\left(\phi_{s}\right)$ while the gas is heated only by the drag interaction and the decrease in gas volume on compaction. Any heat transfer from the solid to low-density gas would be extremely slow and insignificant. Figure 3 from [14] shows the most likely mechanism for energy localization, the localized deformation of the solid. 


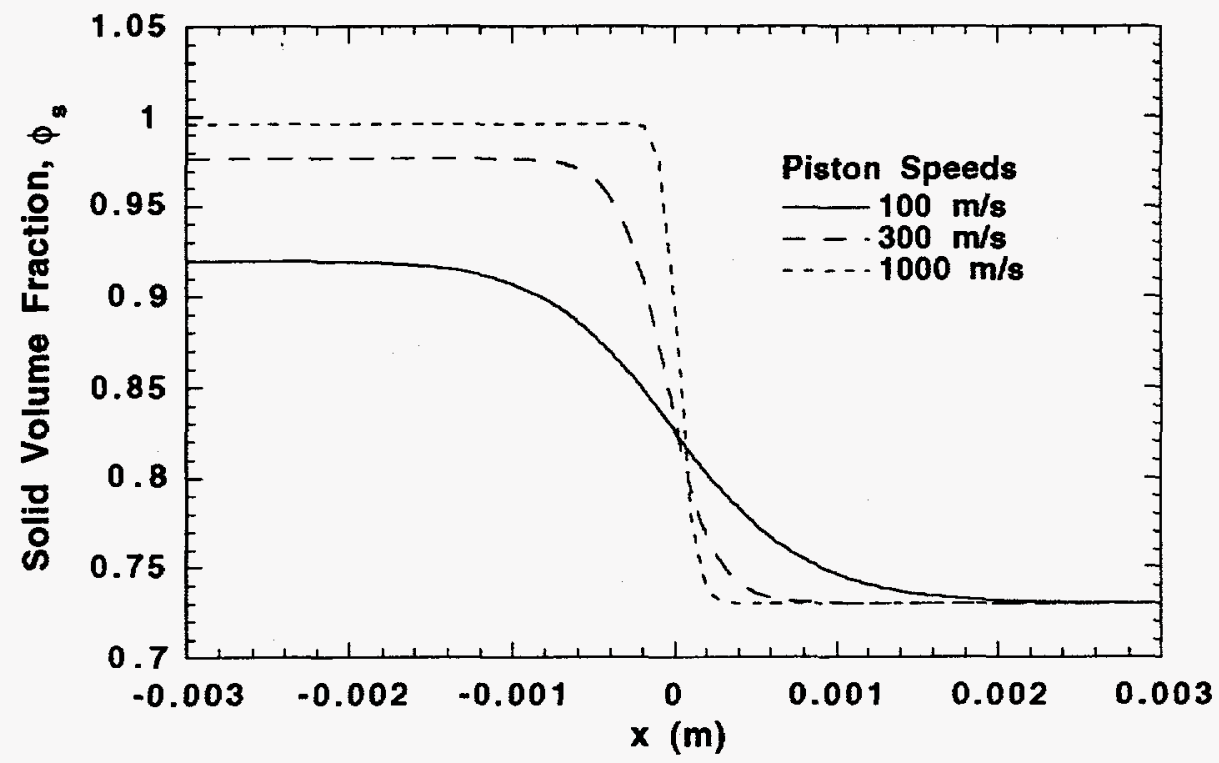

(a)

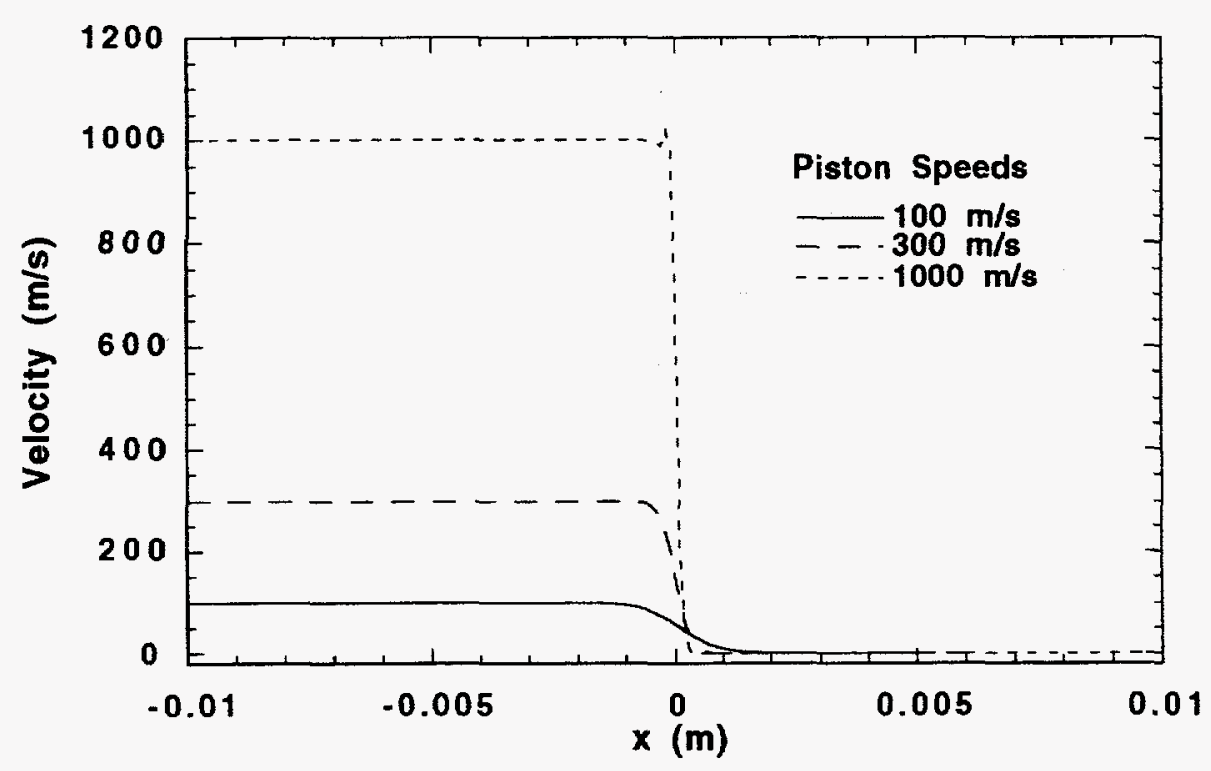

(b)

Fig. 2: The thickness of the compaction wave decreases with increasing piston speed. This property follows directly from Eqs. (7), (13), and (14). The gas phase density is zero in these calculations. 

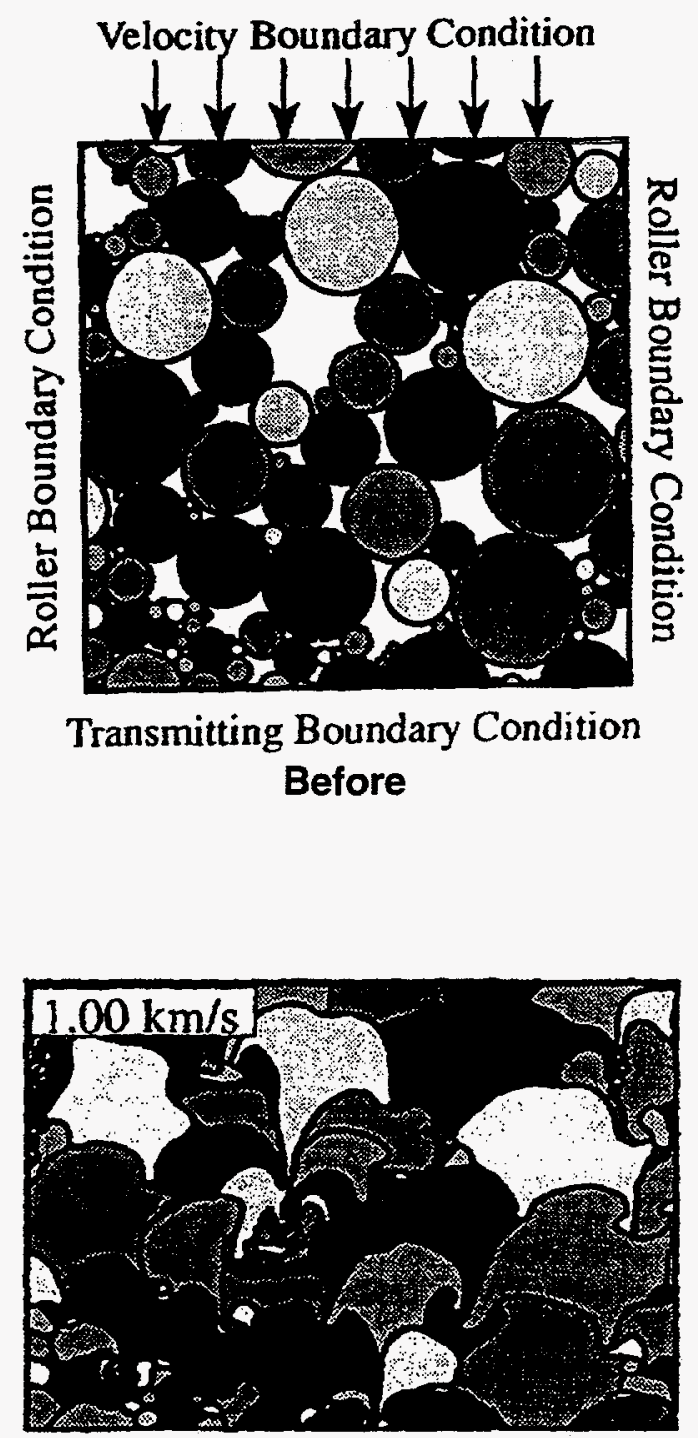

After

Fig. 3: Numerical simulation of stress loading of granular copper. Note the deformation of the regions of the solid particles that border the voids. This figure is reprinted from Benson [14] with permission from the author and the American Institute of Physics.

Of all the exchange laws, the chemical reaction law $C_{s}^{\dagger}$ is the most poorly understood. Equation (10) is a model for the gas phase combustion of HMX. It is a surface burning model for spherical grains of HMX; it describes the sublimation of solid into gas and the subsequent gas phase combustion. Significantly, the burning rate is a function of the gas pressure $P_{g}$. The constants acoef and $b n$ in Eq. (10) were obtained from strand burner experiments performed on HMX. This expression models the principal energy-releasing process in HMX based materials. It is used here and in Baer and Nunziato's work [3].

Hidden in this mostly experimentally measured expression is the ignition factor eti. It is 
Hidden in this mostly experimentally measured expression is the ignition factor eti. It is a switch that is used to turn on the principal energy-releasing reaction; et $i=0$ \{off , et $i=1$ \{on\}. Here we set $e t i=1$ when the mixture pressure, $\phi_{s} P_{s}+\phi_{g} P_{g}$ exceeds $10^{7} \mathrm{~N} / \mathrm{m}^{2}$. In Baer's most recent model, two additional variables and evolution equations are introduced to control ignition and the switching of eti. This ignition model is described in the M-7 Memo dated 10-15-93 which can be found in Appendix A of this report [15]. Since this ignition model is (1) rather ad hoc and (2) calibrated specifically for $\phi_{s}{ }^{\circ}=0.73$ granular HMX, we use the simple pressure ignition switch in our exposition. The success of a DDT model is largely related to how well we do at modeling the ignition process that controls eti. Work on this issue is ongoing and is the current focus of this program.

In order to study these equations, Baer wrote a one-dimensional, two-phase, two-velocity and one-material code that employs a Method-of-Lines (MOL) algorithm. In the next section, we discuss the properties of the solutions to the BN model. These results were obtained both numerically and analytically.

\subsection{Properties of the BN Model}

The BN equations, Eqs. (1)-(7) constitute a system of hyperbolic PDEs. The mathematical properties of these equations have been studied by Embid and Baer [16] and Embid, Hunter and Majda [17]. When all the interaction terms are set to zero (i.e., $C_{s}^{\dagger}=0, P_{g}\left(\phi_{s}\right)_{x}=0$, $\delta=0, \mathcal{F}=0, \mathcal{H}=0$ ), then the gas and solid phases support noninteracting gas and solid waves. The physically most interesting noninteracting flows correspond to the pure phase limits $\rho_{g} \rightarrow 0$ (a porous solid with no gas) and $\phi_{s} \rightarrow 0$ (a pure gas with no solid). Equations (1)-(6) do not automatically limit to these cases by taking $\rho_{g}=0$ or $\phi_{s}=0$. In addition, one must set to zero the compaction work term Eq. $(25 \mathrm{a}, \mathrm{b})$, the heat transfer $\mathcal{H}$, and the drag coefficient $\delta$. This points out a deficiency of the Baer model, the failure to limit properly. The limit $\phi_{s} \rightarrow 0$, yields the equations of inviscid gas dynamics. When $\rho_{g} \rightarrow 0$, Eqs. (1), (3), (5), (7), (8a or $8 \mathrm{~b}),(9)$, and (25b) describe a Herrmann $P-\alpha$ porous solid

$$
\begin{aligned}
\left(\phi_{s} \rho_{s}\right)_{t}+\left(\phi_{s} \rho_{s} V_{s}\right)_{x} & =0 \\
\left(\phi_{s} \rho_{s} V_{s}\right)_{t}+\left(\phi_{s} \rho_{s} V_{s}^{2}+\phi_{s} P_{s}\right)_{x} & =0 \\
\left\{\phi_{s} \rho_{s}\left[e_{s}\left(P_{s}, \rho_{s}\right)+V_{s}^{2} / 2\right]\right\}_{t}+\left\{\phi_{s} \rho_{s} V_{s}\left[e_{s}\left(P_{s}, \rho_{s}\right)+V_{s}^{2} / 2\right]+\phi_{s} P_{s} V_{s}\right\}_{x} & =0
\end{aligned}
$$

and

$$
\left(\phi_{s}\right)_{t}+V_{s}\left(\phi_{s}\right)_{x}=\mathcal{F}
$$

with $\mathcal{F}$ given by Eq. (9). Rewriting these equations and for the moment not neglecting the gas pressure, easily yields

$$
\frac{D_{s} P_{s}}{D t}-\mathcal{C}_{s}^{2} \frac{D_{s} \rho_{s}}{D t}=\left\{\frac{\beta_{s}\left(\phi_{s}\right) \mathcal{F}}{\phi_{s} \rho_{s}\left(\frac{\partial e_{s}}{\partial P_{s}}\right)_{\rho_{s}}}\right\} \text { or }\left\{\frac{\left(P_{s}-P_{g}\right) \mathcal{F}}{\phi_{s} \rho_{s}\left(\frac{\partial e_{s}}{\partial P_{s}}\right)_{\rho_{s}}}\right\}
$$

where $\mathcal{C}_{\boldsymbol{s}}$ is the solid phase sound speed and

$$
\frac{D_{s}}{D t}=\frac{\partial}{\partial t}+V_{s} \frac{\partial}{\partial x}
$$


is the substantial derivative for the solid. The term

$$
\frac{\beta_{s}\left(\phi_{s}\right) \mathcal{F}}{\phi_{s} \rho_{s}\left(\frac{\partial e_{s}}{\partial P_{s}}\right)_{\rho_{s}}}>0 \text { or } \frac{\left(P_{s}-P_{g}\right) \mathcal{F}}{\phi_{s} \rho_{s}\left(\frac{\partial e_{s}}{\partial P_{s}}\right)_{\rho_{s}}}>0
$$

is similar to the thermicity parameter from detonation theory [18]. Since $\mathcal{F} \geq 0$, the source in Eq. (30a, b) plays much the same role as does an exothermic reaction in detonation theory. Equation $(32 \mathrm{a}, \mathrm{b})$ produces heating of the solid in addition to the heating produced by shocks.

Computations were done on these equations with the "September-BKS" code [19]. This code, developed by Stewart and Xu at the University of Illinois, Kapila at RPI, and Bdzil and Son at Los Alamos, solves a special limit of the BN equations called the BKS model. It solves a system of conservation laws, including source terms. The solution is obtained by using time splitting to separately advance the source terms and hydrodynamics. For example, the solid mass equation is solved by first advancing the hydrodynamics

$$
\left(\phi_{s} \rho_{s}\right)_{t}+\left(\phi_{s} \rho_{s} V_{s}\right)_{x}=0
$$

using a second-order, MacCormack's predictor-corrector algorithm, and then advancing the source terms

$$
\left(\phi_{s} \rho_{s}\right)_{t}=C_{s}^{\dagger}
$$

by subcycling in time and using central differencing where required. There are three versions of the code: (1) "September-BKS" solves the 6-equation BKS model, (2) "September-2V" solves the 7-equation BN model, and (3) "September-1V" solves the reduced 7-equation, single velocity, large-drag model. Models (1) and (3) will be described in subsequent sections.

The results for a simple, solid-only compaction wave governed by Eqs. (26)-(29) are displayed in Figure 2. The solid EOS described previously was used, for a system with $\phi_{s}^{o}=0.73$ and piston speeds of $100 \mathrm{~m} / \mathrm{s}, 300 \mathrm{~m} / \mathrm{s}$ and $1000 \mathrm{~m} / \mathrm{s}$, respectively. In all cases the waves were traveling steadily. The state of compaction for the $1000 \mathrm{~m} / \mathrm{s}$ wave is $\phi_{s}=0.996$. As expected, for the rate given by Eq. (9), the thickness of the compaction wave decreases as it goes from a weak, subsonic, dispersive wave for the $100 \mathrm{~m} / \mathrm{s}$ piston to a supersonic "shock" for the $1000 \mathrm{~m} / \mathrm{s}$ piston. The temperatures achieved by the solid, initially at $300 \mathrm{~K}$, were $305 \mathrm{~K}, 336 \mathrm{~K}$ and $620 \mathrm{~K}$, respectively.

In the ambient condition, the voids in a porous solid are filled with low-density gas (air). To study the role (if any) that this low density gas may play in the compaction process, we also solved Eqs. (1)-(9) using "September-2V" for the case where $C_{s}^{\dagger}=0$, Eq. (25b) as the model for compaction work and no heat transfer $\mathcal{H}=0$ between the phases. The analog of Eq. $(30 a, b)$ for the gas phase is

$$
\frac{D_{g} P_{g}}{D t}-\mathcal{C}_{g}^{2} \frac{D_{g} \rho_{g}}{D t}=\left\{\frac{\left(P_{s}-\beta_{s}\left(\phi_{s}\right)-P_{g}\right) \mathcal{F}}{\phi_{g} \rho_{g}\left(\frac{\partial e_{g}}{\partial P_{g}}\right)_{\rho_{g}}}+\frac{\delta\left(V_{s}-V_{g}\right)^{2}}{\phi_{g} \rho_{g}\left(\frac{\partial e_{g}}{\partial P_{g}}\right)_{\rho_{g}}}\right\} \quad \text { or } \quad\left\{\frac{\delta\left(V_{s}-V_{g}\right)^{2}}{\phi_{g} \rho_{g}\left(\frac{\partial e_{g}}{\partial P_{g}}\right)_{\rho_{g}}}\right\} \text {, }
$$

where $\mathcal{C}_{g}$ is the gas phase sound speed and $\frac{D_{g}}{D t}$ is the substantial derivative for the gas. When the right hand side of Eq. $(35 \mathrm{a}, \mathrm{b})$ is zero, the flow in the gas (except for shocks) is 
isentropic. What the source term indicates is that the drag interaction enters as a positive thermicity. Thus irrespective of the sign of $\left(V_{s}-V_{g}\right)$, the drag between the solid and gas heats the gas. This drag-related heating appears in the gas energy equation, Eq. (35a, b), but not in the solid energy equation, Eq. $(30 \mathrm{a}, \mathrm{b})$. The asymmetry is related to the form of the source term in Eqs: (5)-(6), i.e., $\delta\left(V_{s}-V_{g}\right) V_{s}$. The motivation for this choice is related to the physical picture of the interaction adopted by BN. They picture a slow, Stokes-like flow of a deformable, viscous gas over a rigid, solid sphere. In such an interaction, all of the fluid mechanics is assigned to the gas, which for a Stokes flow corresponds to a balance of viscous and pressure forces and the neglect of inertial forces. Consequently, the gas and solid exchange momentum, but only the gas experiences a change in its internal energy.

Typically, the gas remains shockless in many problems we consider. Thus, the amount of gas heating is principally determined by $\delta\left(V_{s}-V_{g}\right)^{2}$. As we show in Section 4 and Appendix $\mathrm{B}$ of this report, this heating takes place in a narrow region near the leading edge of the wave. In many situations, the amount of heating is small. Specializing Eqs. (20)-(21) to an ideal gas by taking

$$
R_{1}=R_{2}=0 ; \quad h_{r e l}=-C_{v g} \star T_{g}{ }^{o},
$$

and computing the $1000 \mathrm{~m} / \mathrm{s}$ piston example, yields a gas temperature of $\approx 820 \mathrm{~K}$. This is almost entirely a result of the adiabatic compression of the gas

$$
T_{g}=T_{g}{ }^{o}\left(\rho_{g} / \rho_{g}{ }^{o}\right)^{\omega}
$$

The relatively small contribution from the drag interaction term is consistent with the findings on drag interaction across shocks discussed in Appendix B [7]. The final state of compaction is essentially the same as that found when no gas is present in the pores (i.e., $\phi_{s}=0.996$ ); the gas pressure is two orders of magnitude lower than the solid pressure. Owing to the large value of the drag, the solid and gas move together. Thus it would appear that the gas does not produce "hotspots" directly, but rather the deformation of the solid produced by the presence of voids causes them to form. The profiles for this case are well represented by Figure 2 .

We now go on to consider the consequences resulting from a zero drag interaction (i.e., $\delta \rightarrow 0$ ). The problem we examine is a simple compaction wave. This problem was first studied by Kapila and Bdzil during the summer of 1992 [20]. They considered the limit of no reaction, no heat transfer, no drag and no compaction work (i.e., $C_{s}^{\dagger}=0, \mathcal{H}=0, \delta=0$ and Eq. (25b)). That is, the gas energy equation, Eq. (35b) is source term free. Here the coupling of the phases is through the porosity variable $\phi_{s}$. For the purposes of this example a polytropic gas and Tait solid were assumed (see Appendix B for descriptions of these EOSs).

The piston speed was $100 \mathrm{~m} / \mathrm{s}$ and a standard temperature and pressure (STP) ideal gas was used. These results were obtained using the two-velocity code "September-2V." A late time snapshot showing the solid and gas particle velocities and $\rho_{g} \phi_{g}$ are shown in Figure 4. With the absence of the aforementioned exchange terms, the compaction of the solid by the piston forces the gas into the uncompacted bed much as squeezing on a toothpaste tube forces out the toothpaste. The compaction front in the solid acts like a virtual piston that drives the gas. In turn, as the pressurized gas moves ahead of the compaction front, it tends to increase the porosity. This effect is small owing to the low initial gas density. 
Significantly, when there is no interphase drag, the gas can permeate a long distance into the porous solid; this gives an upper bound on the permeation. The theoretical results [20] are in good agreement with the numerical results shown in Figure 4.

The example shown in Figure 4 corresponds to a case where the virtual "piston" speed (i.e., the speed of the compaction wave) is slower than the gas dynamic wave that it supports in the gas; the flow behind the shock is subsonic in the gas. By increasing the density of the initially quiescent gas by a factor of 10 , we can make the speed of the "piston" (i.e., the compaction wave) faster than that of the gas dynamic wave in the gas; the compaction wave is a supersonic piston for the gas. Such an example is shown in Figure 5. The compaction wave moves through the gas at supersonic speeds, accelerating and pressurizing the gas as a result of the compaction of the solid. The change in gas density sets the value of the gas particle velocity at a value below that of the solid; without drag interaction the solid and gas move at different speeds. Trailing behind this supersonic, compaction supported wave in the gas is a gas dynamic shock supported by the real piston. Interestingly enough, the pressure snapshot shown in Figure 5 resembles what Baer misidentified as a permeation layer in his pressure snapshot that is shown in Figure 6 [3].

As the above examples show, the wave interactions that can take place in a two phase material can be complex. For the example considered above, the compaction term is the source (see Eq. (29)) that couples the two phases together and results in a flow where the solid is the driver, and the gas is the driven material. This all happens without interphase drag. The richness in the wave structure of two phase models brings both advantages and disadvantages. More phenomena can be modeled. However, a higher-level understanding of gas dynamics is required to keep from misidentifying those phenomena. To treat a rich, complex model as simply a black box is a mistake.

The consequences in a combustion problem like DDT of having "hot," dense gas permeating a long distance into the solid, cool HMX-like material are clear; the combustion would be accelerated. In fact as Figure 6 shows, Baer has long argued for the importance of this macroscopic permeation for accelerating deflagration towards detonation [3]. To this end, Shepherd and Begeal [21] did experiments to measure the permeability of porous beds of inerts and HMX over a range of porosities. A schematic of their experimental setup is shown in (a) of Figure 7. In this nominally isothermal experiment, the gas chamber was initially at 30,000 psi and a temperature of $300 \mathrm{~K}$. Equation (12) was determined in this study. Using the parameter values for granular HMX listed earlier $\left(a p=7.5 \times 10^{-5} \mathrm{~m}\right)$ and neglecting the Reynold's number dependence in Eq. (12), yields

$$
\delta=5.67 \times 10^{5} / \phi_{g}{ }^{4.5} \mathrm{~kg} / \mathrm{m}^{3} / \mathrm{s} .
$$

For values of $\phi_{g}$ typical for damaged explosive, $\phi_{g}{ }^{\circ}=0.27$ it follows that

$$
\delta=2.05 \times 10^{8} \mathrm{~kg} / \mathrm{m}^{3} / \mathrm{s} .
$$

Neglecting compaction and reaction in the gas momentum and reaction in the gas momentum equation (i.e., in Eq. (4)) and assuming that the flow in the gas and solid are not equal but uniform,

$$
V_{g}>0 ; \quad V_{s}=0
$$




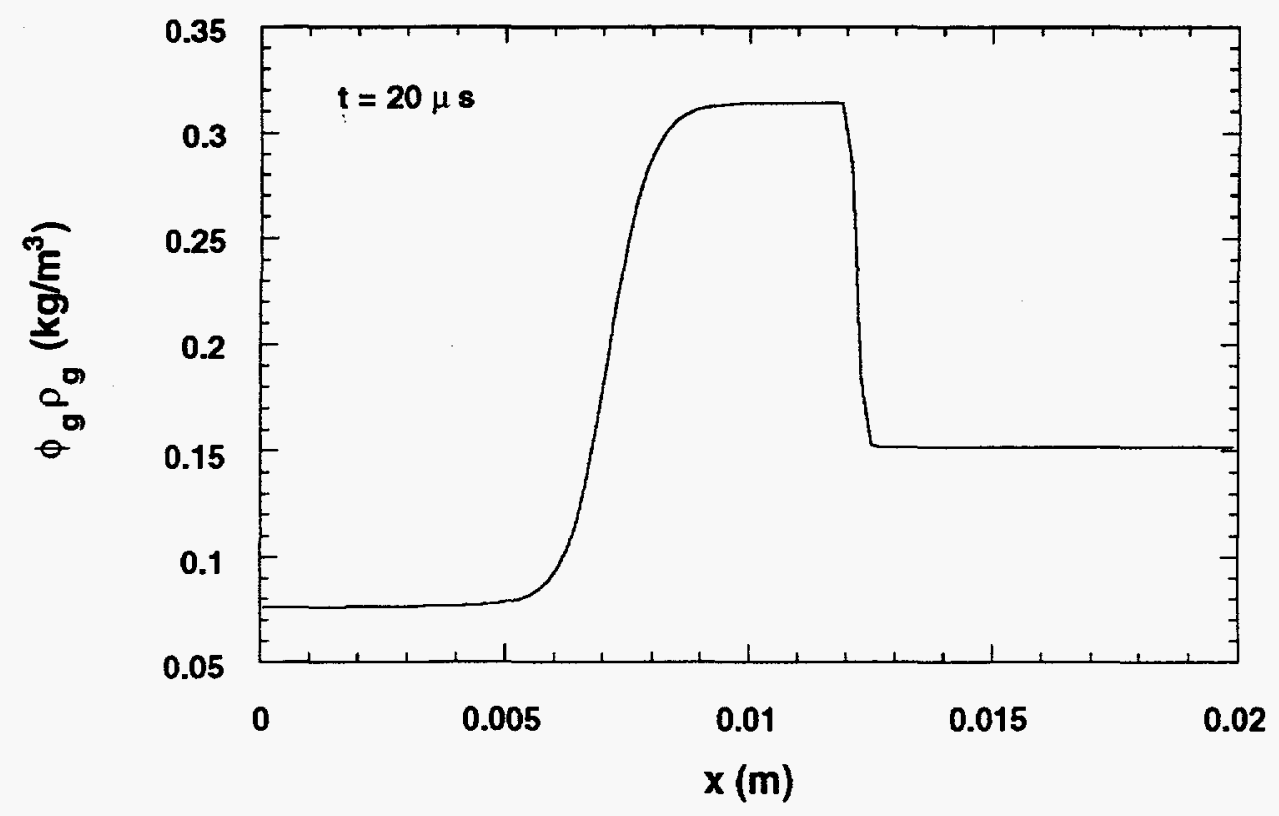

(a)

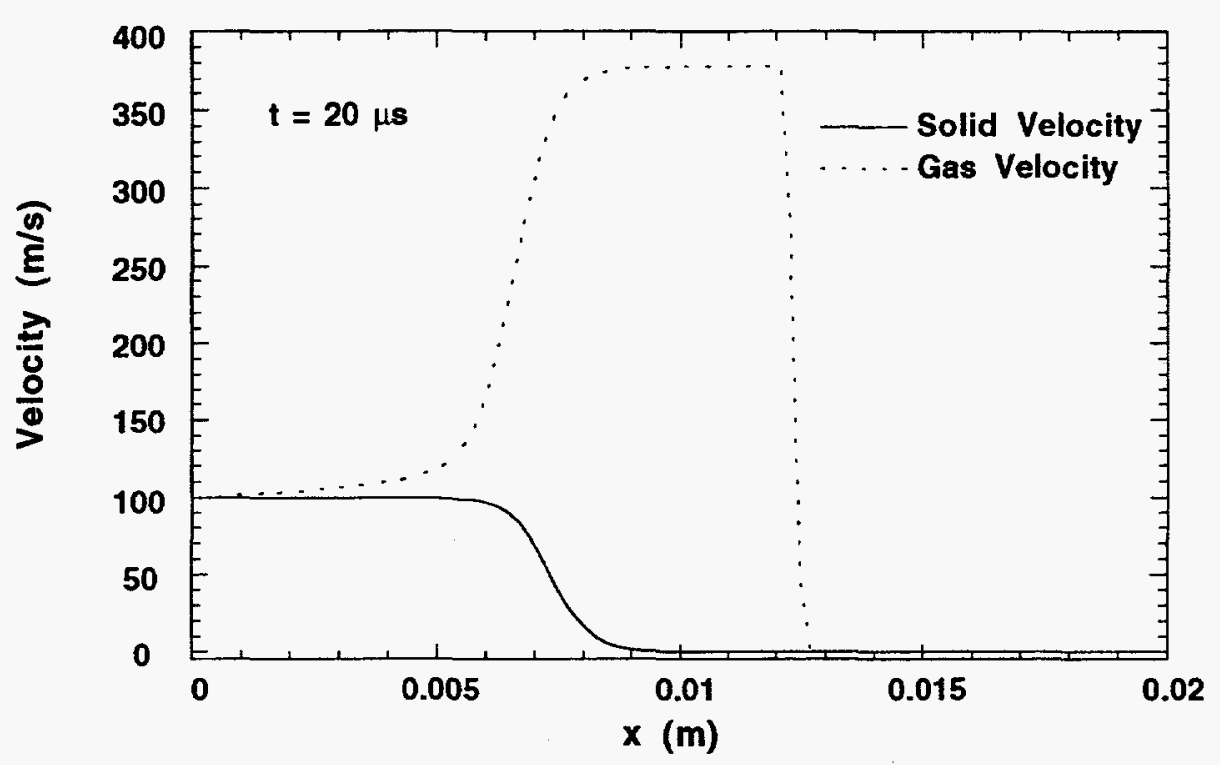

(b)

Fig. 4: The zero-drag limit: low gas density. Snapshots at $t=20 \mu s$ of the gas density, $\phi_{g} \rho_{g}$ and the particle velocities show that the gas is pumped from the pores and moved ahead of the compaction wave. The solid compaction wave acts as a $400 \mathrm{~m} / \mathrm{s}$ virtual piston. The virtual piston speed is less than the sound speed in the gas. The zero drag limit defines the maximum gas "permeation" distance. 


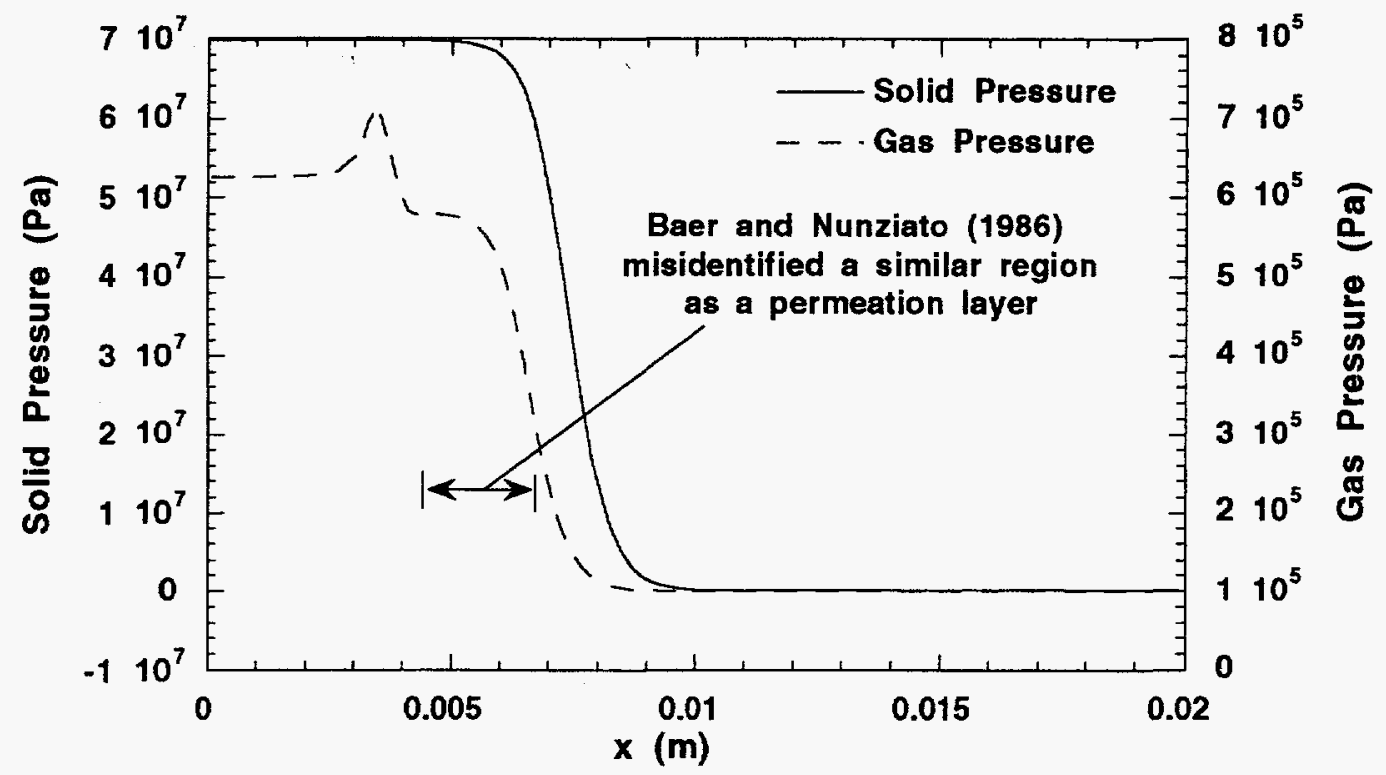

(a)

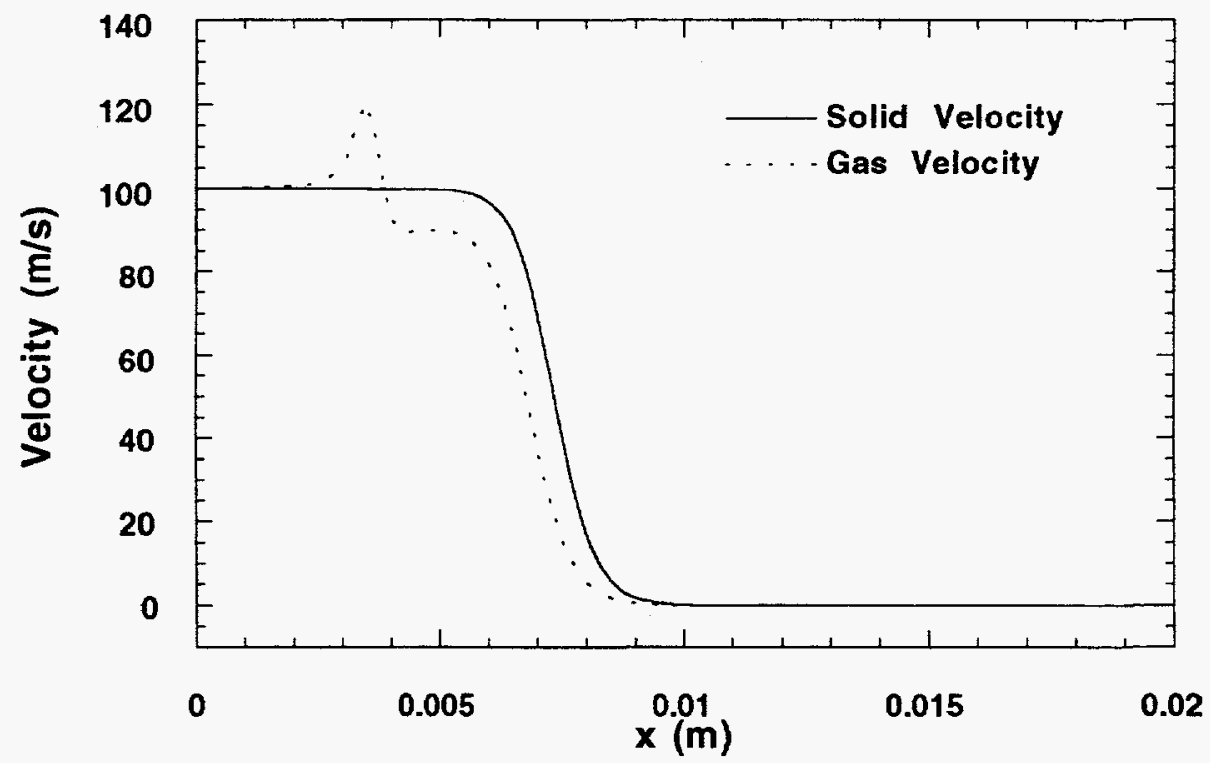

(b)

Fig. 5: The zero-drag limit: high gas density. Snapshots at $t=20 \mu s$ of the gas pressure, $P_{g}$ and the particle velocities (upper-solid, lower-gas) show that the solid compaction wave acts like a supersonic virtual piston that drives the gas. The trailing shock in the gas is driven by the real piston in the problem. 


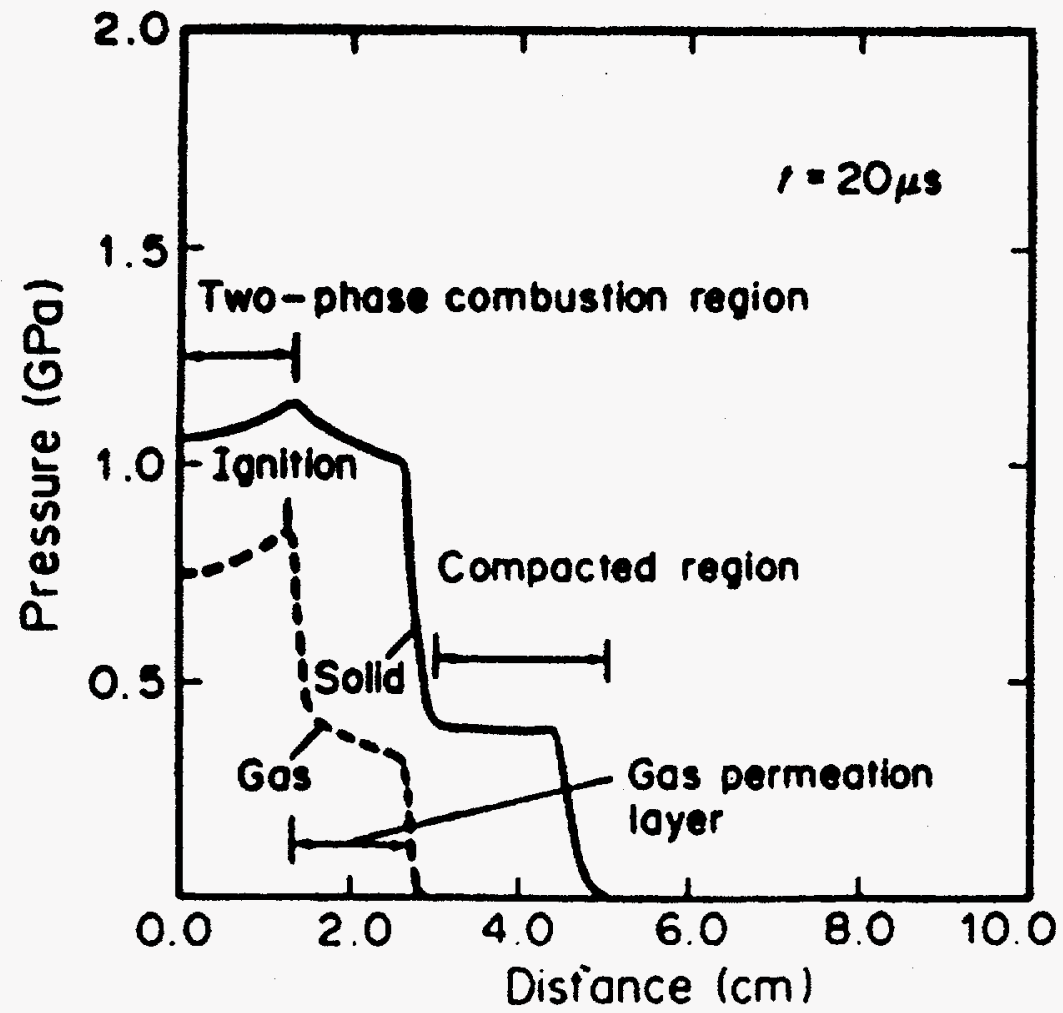

Fig. 6: Baer argues that the gas permeation layer is $1 \mathrm{~cm}$ wide at a problem time of 20 $\mu s$. The zero-drag, supersonic wave phenomenon described in Figure 5 is responsible for his observation. This figure is reprinted from [3] with kind permission from Elsevier Ltd, The Boulevard, Langford Lane, Kidlington OX5 1GB, UK. 


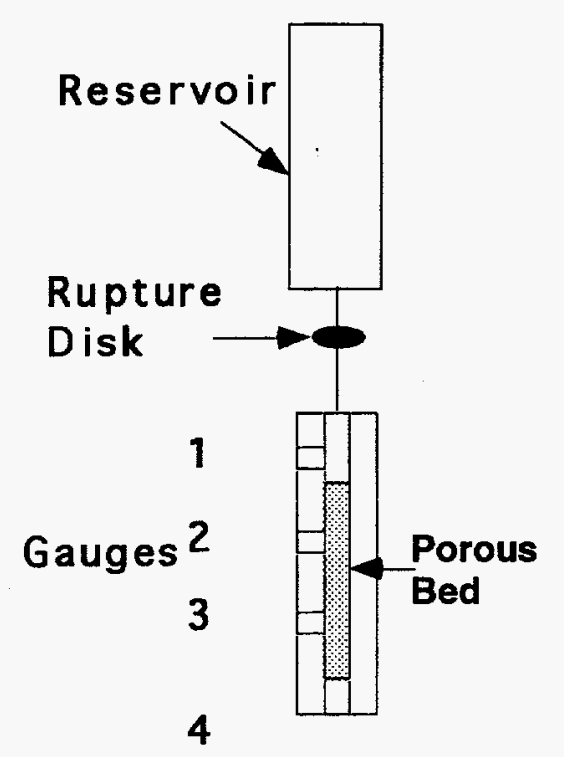

(a)

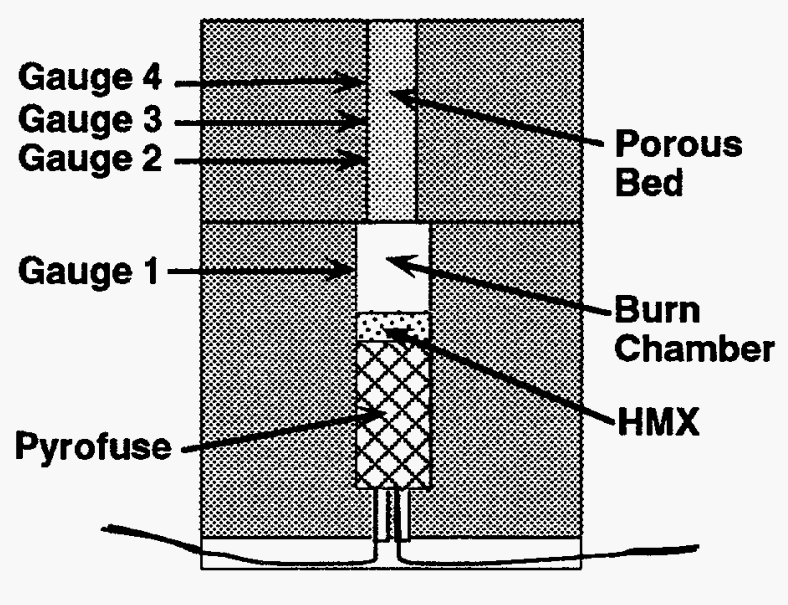

(b)

Fig. 7: Conceptual schematics of the gas permeation experiments.

it follows that the e-folding time for the decay of $V_{g}$ to zero with $V_{s}$ constrained to zero is

$$
\left(\phi_{g} \rho_{g}\right) / \delta \approx 10^{-7} \mathrm{~s}
$$

for $\left(\phi_{g} \rho_{g}\right) \approx 10 \mathrm{~kg} / \mathrm{m}^{3}$. This is a very short time on the time scale of our problems; permeation of gas any significant distance through the bed would be very slow. As the gas density increases, the above decay time becomes longer implying that the gas can travel farther. Thus hot, dense, high-pressure combustion products could permeate further into the bed. However, under these circumstances the drag coefficient could also become larger.

To check this, Asay, Funk and Laabs [22] did some permeation experiments using higher gas pressures (90,000 psi) and higher gas temperatures (4000 K). An experimental geometry similar to that shown in (b) of Figure 7 was used. The high-pressure, high-temperature gas was generated by burning HMX powder in the gas chamber. Instead of having a permeation tube that was sealed at its right end as was the case in Shepherd's experiments, the tube end was open to the atmosphere. Some of Asay's results are shown in Figure 8. The porous bed consisted of $\mathrm{SiC}$, which, it was observed, didn't compact in the course of the experiment. The gas pressure was measured at the four stations shown in (b) of Figure 7; the traveling gas-permeation front had a sharp face. The measured front velocity was a nearly constant $V_{\text {perm }}=5 \mathrm{~m} / \mathrm{s}$; one-hundred times slower than the speed of sound in air. Clearly, a $5 \mathrm{~m} / \mathrm{s}$ permeation wave attached to a compaction wave moving at $400 \mathrm{~m} / \mathrm{s}$ in a supporting solid particle flow of $100 \mathrm{~m} / \mathrm{s}$ is a nonissue

$$
V_{\text {comp }} \gg V_{s}+V_{\text {perm }}
$$

If any preconditioning occurs in the bed, the agent is the compaction wave, not the permeating gas. This is the permeation rate in $\phi_{s}=0.73$ granular HMX. Once the bed is 


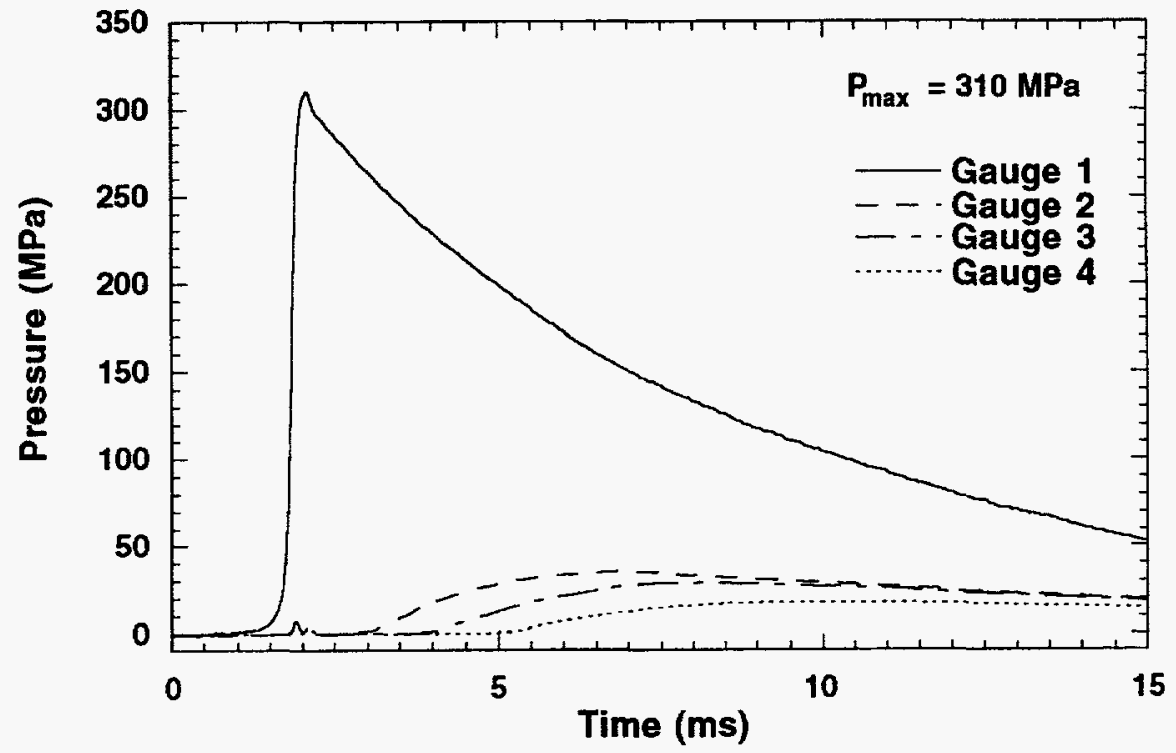

Fig. 8: High-pressure, high-temperature $(4000 \mathrm{~K})$ gas permeation into $\phi_{s}=0.74 \mathrm{SiC}$ porous bed. The pressure at four locations is shown (1) in the burn chamber, (2) $0.9 \mathrm{~cm},(3) 1.4 \mathrm{~cm}$, and (4) $1.9 \mathrm{~cm}$ down the porous bed, respectively. The mean permeation rate is $V_{\text {perm }}=5$ $\mathrm{m} / \mathrm{s}$. 
compacted, the permeation rate is lower still. In order for the gas permeation to be important the porosity of the bed would need to be very high, say $\phi_{s}<0.5$ as compared to the ambient value $\phi_{s}{ }^{\circ}=0.73$. At this point the combustion is well along (see Eq. (7)) and is sustainable without the aid of macroscopic gas permeation.

Shepherd's experiment was carried out under isothermal conditions. The hot combustion gas used in Asay's experiments leads to a nonisothermal environment with heat loss from the gas to both the combustion chamber and the porous bed. Thus, in order to do a quantitative determination of the permeability constants $\kappa_{1}$ and $\kappa_{2}$ of Eq. (12) from Asay's experiments, the heat transfer needs to be modeled. We modeled the experiments using the $\mathrm{BN}$ equations given earlier, with $\phi_{s}(x)$ prescribed according to

$$
\phi_{s}(x, t)=\phi_{s}^{o} \times H\left(x-x_{i}\right)
$$

(i.e., the $\mathrm{SiC}$ was assumed to not compact) where $\phi_{s}{ }^{o}=0.74, H()$ is the Heavyside function, $x_{i}$ is the interface location, with SiC occupying $x>x_{i}$. Substituting Eq. (42) into the "nozzling" term, Eq. (23a, b), leads to a delta function source, $\delta_{f}\left(x-x_{i}\right)$ at the interface

$$
P_{g}\left(\phi_{s}\right)_{x}=P_{g} \phi_{s}{ }^{\circ} \times \delta_{f}\left(x-x_{i}\right) .
$$

This leads to a technical problem at the interface. The delta function is more properly called a distribution with the following properties: (1) $\lim _{\left(x-x_{i}\right) \rightarrow 0} \delta_{f}\left(x-x_{i}\right) \rightarrow \infty$ and (2) $\delta_{f}\left(x-x_{i}\right)=0$ for $x \neq x_{i}$. Since $P_{g}$ is not constant across the interface (initially $P_{g}$ is discontinuous), it follows that the integral

$$
\int_{x_{i}-\epsilon}^{x_{i}+\epsilon} P_{g} \phi_{s}^{o} \times \delta_{f}\left(x-x_{i}\right) d x=\text { not defined }
$$

as $\epsilon \rightarrow 0$ unless some further information is provided about $P_{g}$ at the interface. The value that the integral in $\mathrm{Eq}$. (44) assumes controls the solution to this permeation problem; all the gas must pass through the interface under the action of Eq. (44). The procedure by which Eq. (44) is given a value as $\epsilon \rightarrow 0$ is called "regularization." For this problem, we used the regularization described in Appendix A.

In the region $x<x_{i}$, the gas was initially hot, HMX combustion products

$$
\begin{array}{ll}
x<x_{i} \text { at } t=0 & \mathrm{P}_{g}^{o}=2.97 \times 10^{8} \mathrm{~N} / \mathrm{m}^{2} \\
& \mathrm{~T}_{g}^{o}=4000 \mathrm{~K} \\
& \rho_{g}^{o}=168 \mathrm{~kg} / \mathrm{m}^{3}
\end{array}
$$

and

$$
\begin{array}{ll}
x>x_{i} \text { at } t=0 & \mathrm{P}_{g}^{o}=10^{5} \mathrm{~N} / \mathrm{m}^{2} \\
& \mathrm{~T}_{g}^{o}=300 \mathrm{~K} \\
& \rho_{g}^{o}=0.75 \mathrm{~kg} / \mathrm{m}^{3}
\end{array}
$$

The values shown in Eq. (45) were obtained using Vers. 205.11 of the Blake combustion code [23]. Since the compressibility factor, $P_{g} /\left(\rho_{g} R T_{g}\right)$, was not much larger than one, the gas was assumed to be ideal with $\rho_{g}{ }^{\circ}$ obtained by taking 


$$
\begin{aligned}
C_{v g} & =1.9 \times 10^{3} \mathrm{~N} \cdot \mathrm{m} / \mathrm{kg} / \mathrm{K} \\
\omega & =0.23, \quad A=B=0
\end{aligned}
$$

in Eqs. (21)-(22). The SiC was modeled using Eqs. (19)-(20) with

$$
\begin{aligned}
\rho_{s}{ }^{o} & =3120 \mathrm{~kg} / \mathrm{m}^{3}, \quad C_{v s}=6.0 \times 10^{2} \mathrm{~N} \cdot \mathrm{m} / \mathrm{kg} / \mathrm{K} \\
\text { gov } & =5.7 \times 10^{4} \mathrm{~kg} / \mathrm{m}^{3} \\
\text { akto } & =1.0 \times 10^{10} \mathrm{~N} / \mathrm{m}^{2}, \quad a p=22 \mu \mathrm{m} .
\end{aligned}
$$

Since the heat exchange expression, Eq. (15), depends only on the gas thermal conductivity, no information specific to $\mathrm{SiC}$ is required. The heat loss from the gas to the burn chamber walls was modeled with a different multiplier on the heat transfer coefficient using

$$
4.1 \star \rho_{g}^{o} \star C_{v g}\left(T_{g}-300\right) \text {, }
$$

in place of Eq. (15). Clearly, both the chemical reaction and compaction were turned off (i.e., $C_{s}^{\dagger}=0$ and $\mathcal{F}=0$ ). Equation (12) was used for the drag law with $\kappa_{1}=150$ and $\kappa_{2}=0.01$.

Baer's MOL code [24] was used with 100 nodes and Rusinov artificial viscosity coefficient set at a low value, $a r v=0.005$; the normal default value is arv $=0.25$. Since the time advance in the MOL algorithm is not tied to a Courant condition, the time step, $\Delta t$ can be much bigger than the acoustic signaling time. Thus the initial acoustic transients can be resolved at the submicrosecond level, while the nearly quasi-steady, long-time flow can be resolved at the 100 -microsecond scale. This is a particularly important feature in a problem where the overall evolution time is $17 \mathrm{~ms}$. The results of these calculations are shown in Figure 9. The chamber $/ \mathrm{SiC}$ interface is located at $x_{i}=3.76 \mathrm{~cm}$, and the bed is $2.54 \mathrm{~cm}$ long. Part (a) of the figure shows a collection of gas phase pressure snapshots at a sequence of times. Part (b) of the figure shows the gas pressure histories at the gauge locations. The total problem time is $17.5 \mathrm{~ms}$. The agreement of calculation and experiment is fairly good overall. However, the calculated permeation front speed is roughly half that observed in the experiments. This could be related to our calculation not dropping the burn chamber pressure fast enough (i.e., the heat transfer to the walls in the reservoir may be underestimated). Thus, the current permeation parameters (developed for HMX) are about right for granular $\mathrm{SiC}$ of the same initial porosity. From these results it is clear that the interphase drag is both large and well represented by the Eq. (12) parameters.

We had previously observed rather small differences between the gas and solid particle velocities in the simulations that we had done with Baer's MOL code [24]. Using the model reactive problem described in this report (all terms on), we calculated the solution for the $\phi_{s}{ }^{\circ}=0.73$ initial porosity HMX bed driven by a $100 \mathrm{~m} / \mathrm{s}$ piston. The default values, listed in the previous subsection, were used for the parameters. The artificial viscosity, number of nodes and problem time were $a r v=0.25$, nodes $=100$, and $t_{\text {final }}=20 \mu \mathrm{s}$, respectively.

Time snapshots of the results of this calculation are displayed in Figures 10, 11, 12 and 13. A comparison of the solid and gas velocities and $\phi_{s}$ at $t=10 \mu \mathrm{s}$ and $t=18.7 \mu \mathrm{s}$ are 


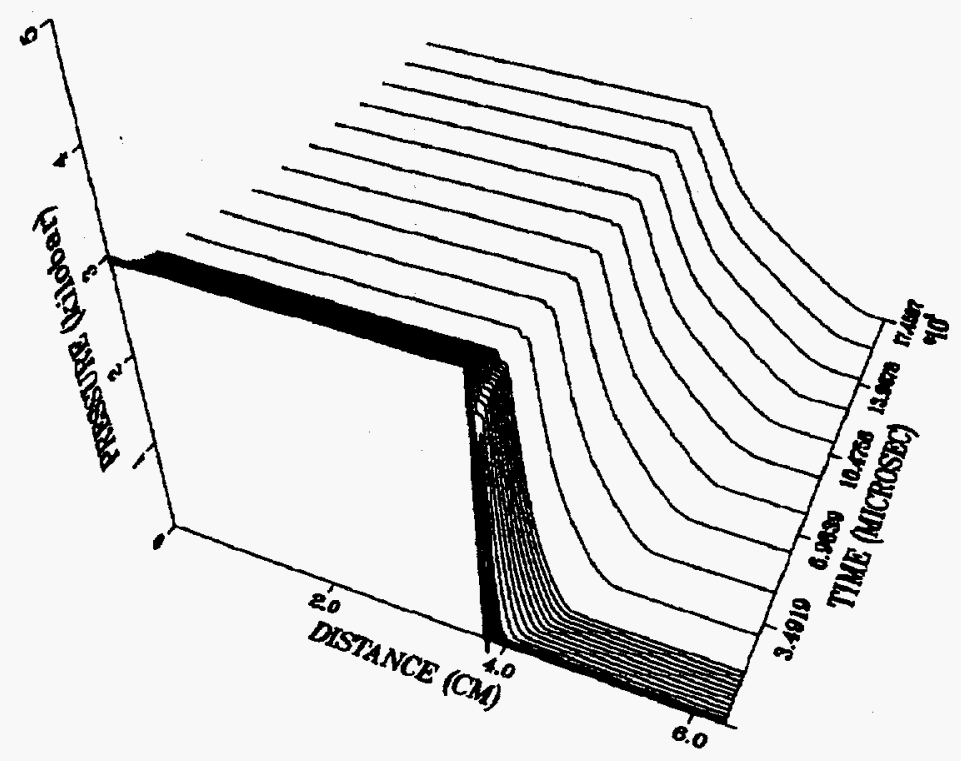

(a)

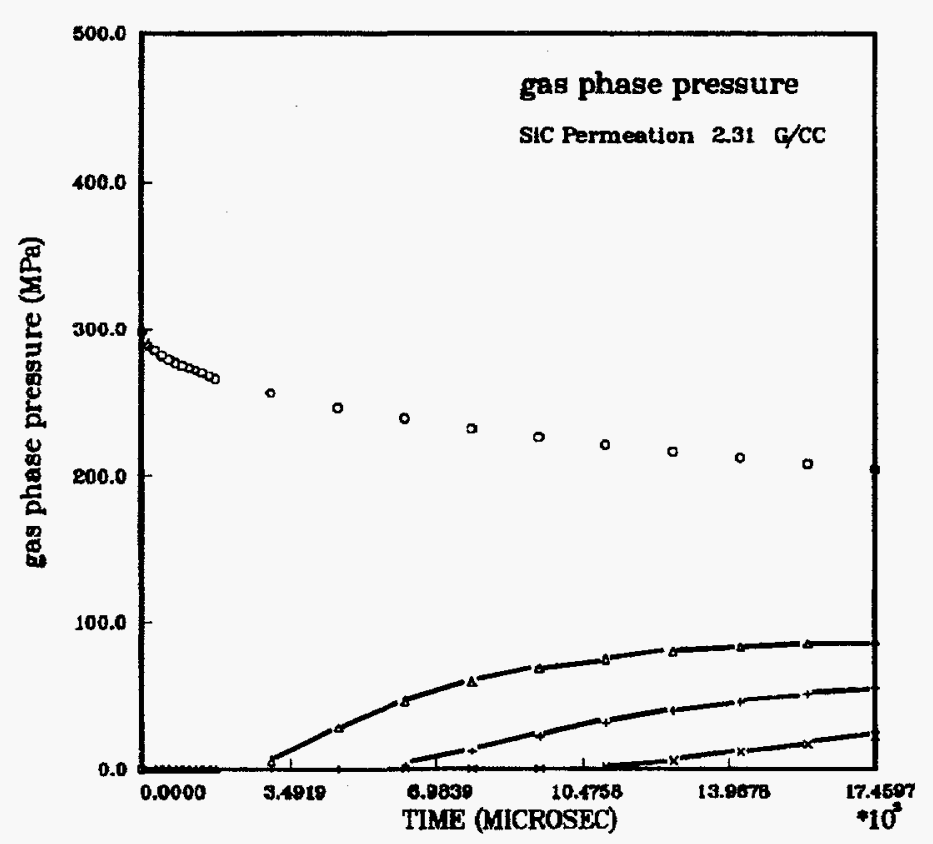

(b)

Fig. 9: Calculation of the Asay SiC bed, gas permeation experiment using Shepherd's lower pressure HMX calibration. The calculated permeation front speed is roughly half as fast as that shown in Figure 8. 


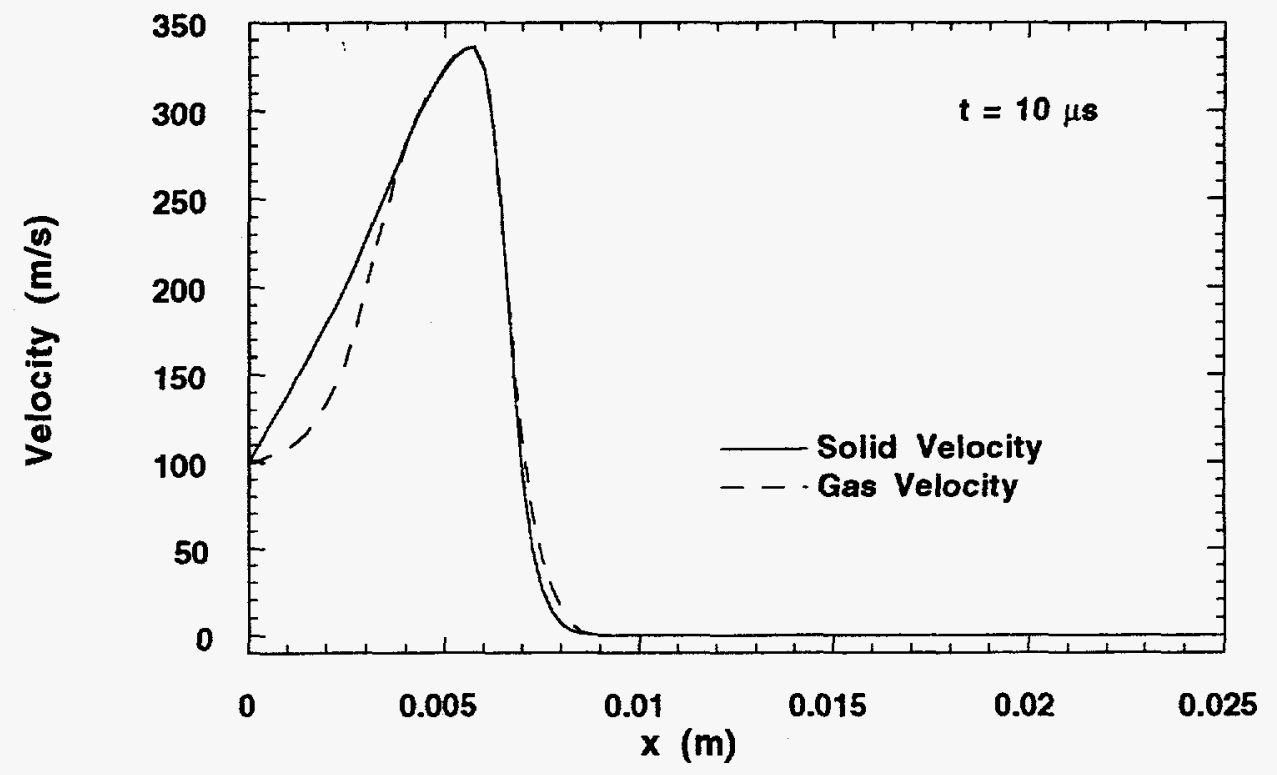

(a)

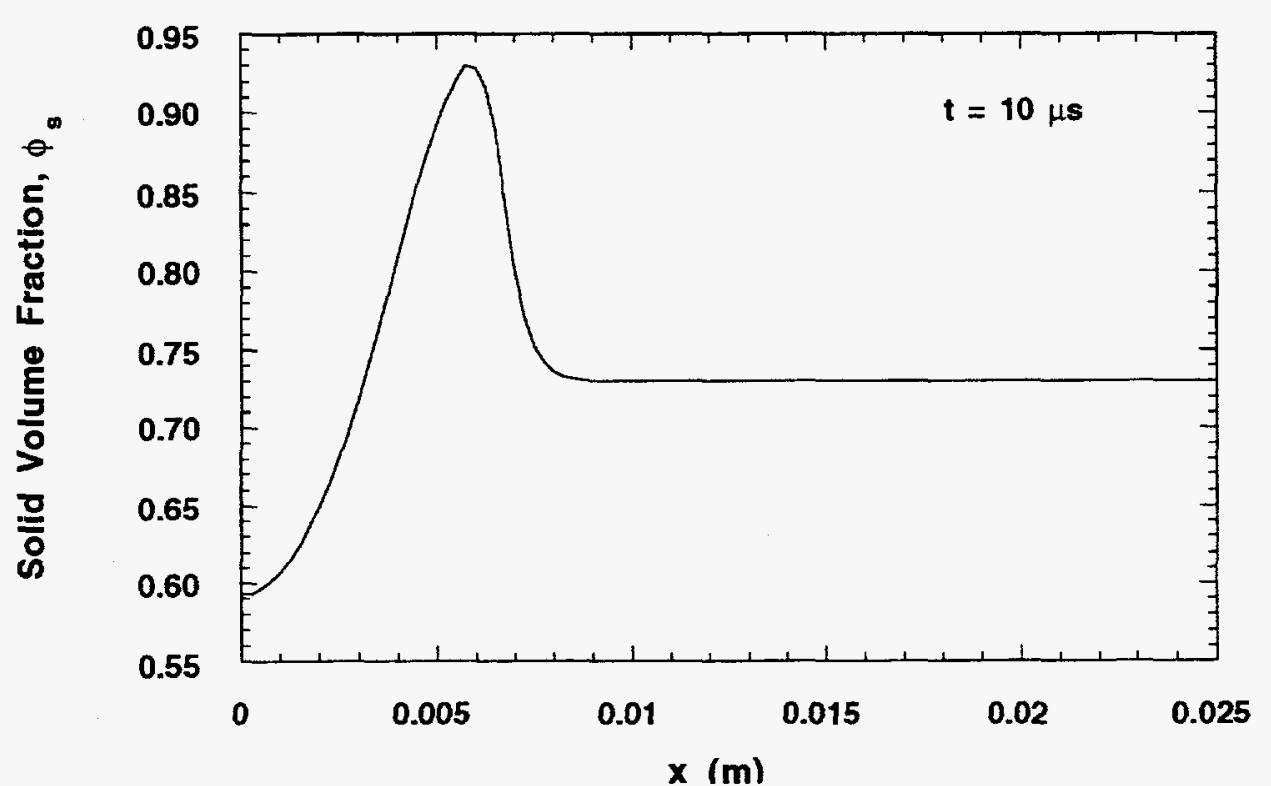

(b)

Fig. 10: A snapshot at $t=10 \mu s$ of the gas (above on the rise and below on the fall) and solid particle velocity are shown in (a) of Figure 10. The $\phi_{s}$ variable is shown in (b) of Figure 10. The velocities overlay when $\phi_{s} \geq 0.8$. 


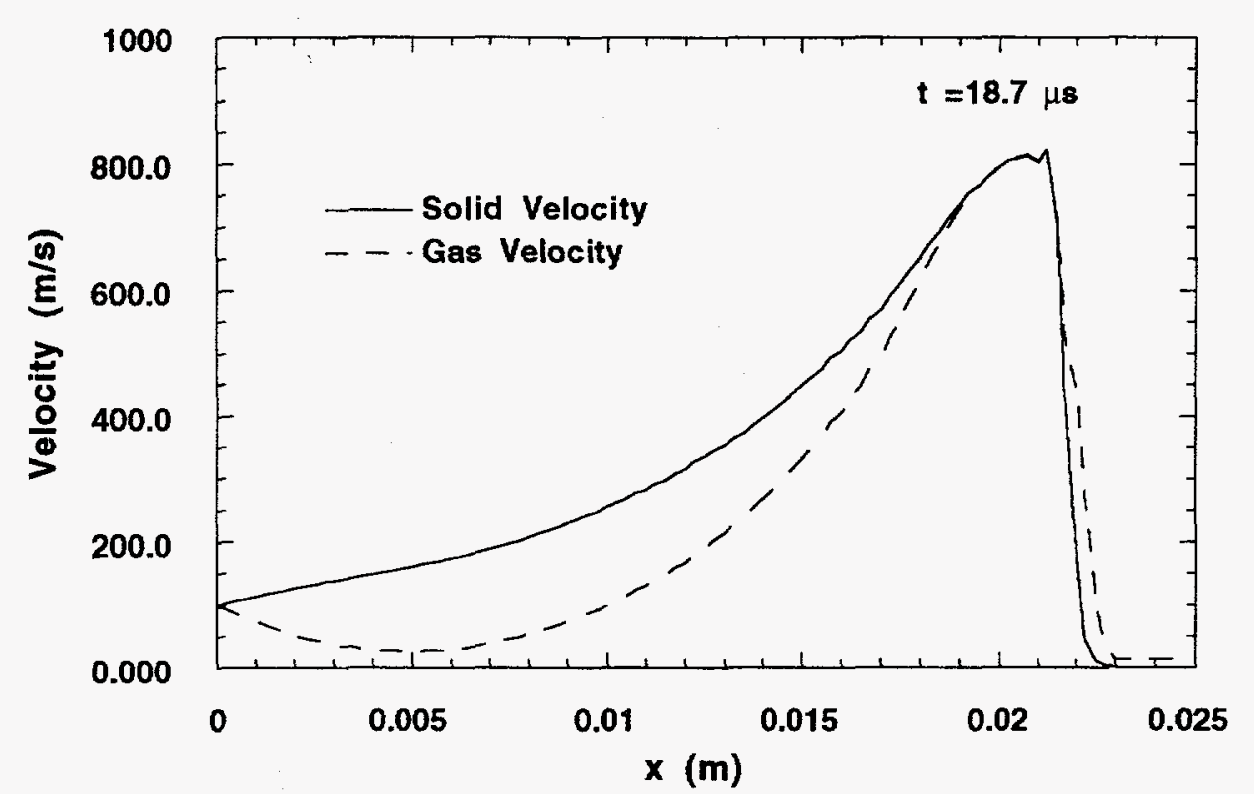

(a)

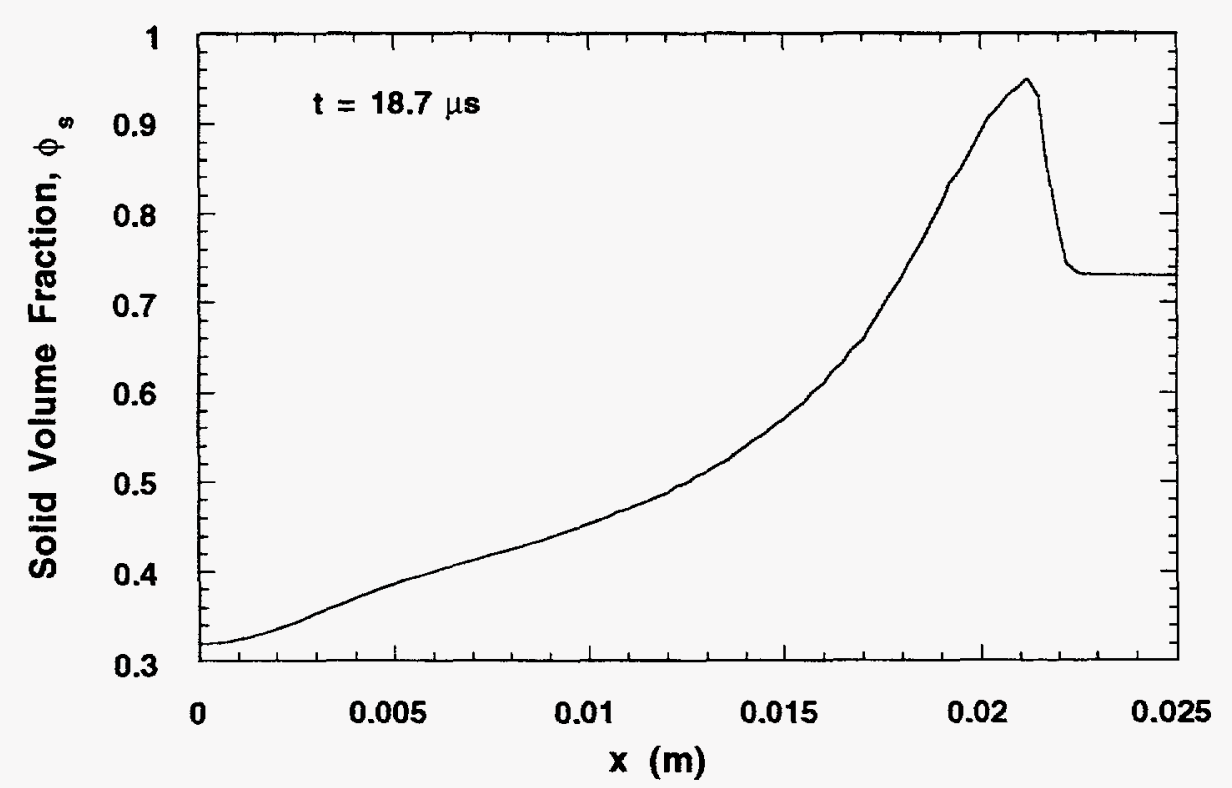

(b)

Fig. 11: A snapshot at $t=18.7 \mu s$ of the gas (above on the rise and below on the fall) and solid particle velocities are shown in (a) of Figure 11. The $\phi_{s}$ variable is shown in (b) of Figure $11 \mathrm{~b}$. The velocities overlay when $\phi_{s} \geq 0.8$. 


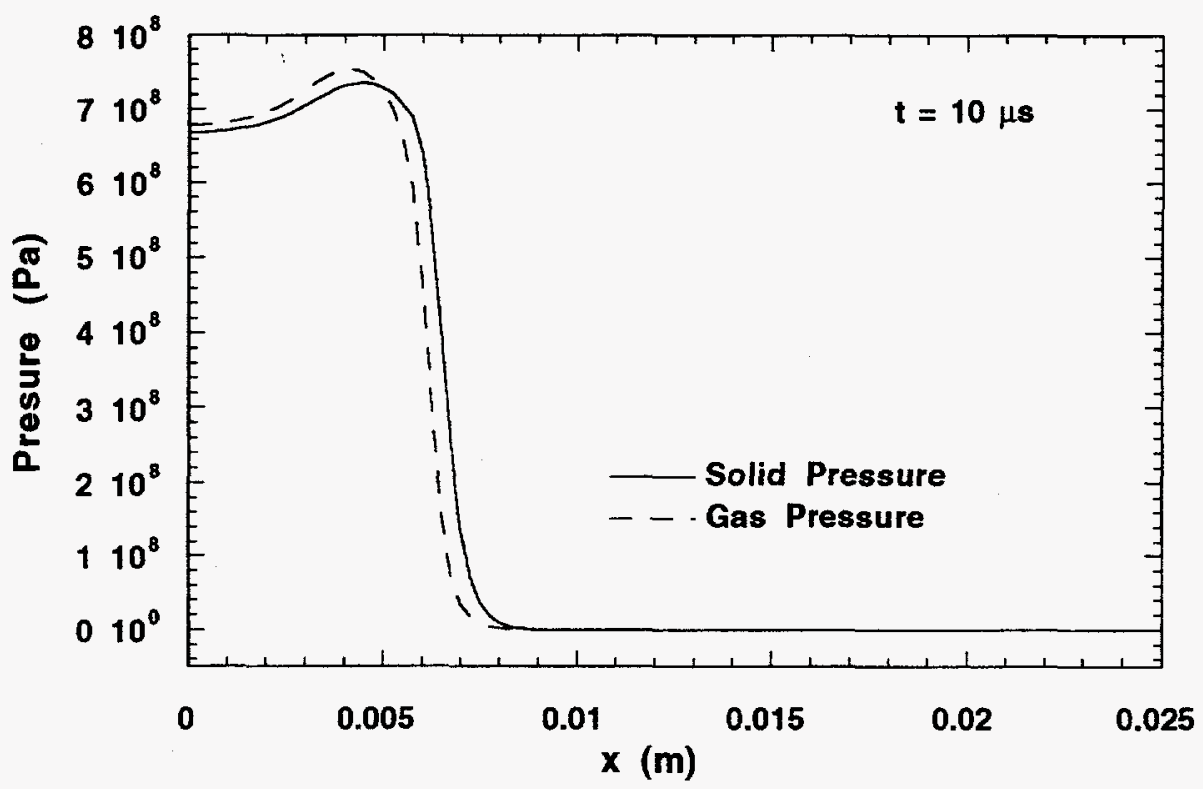

(a)

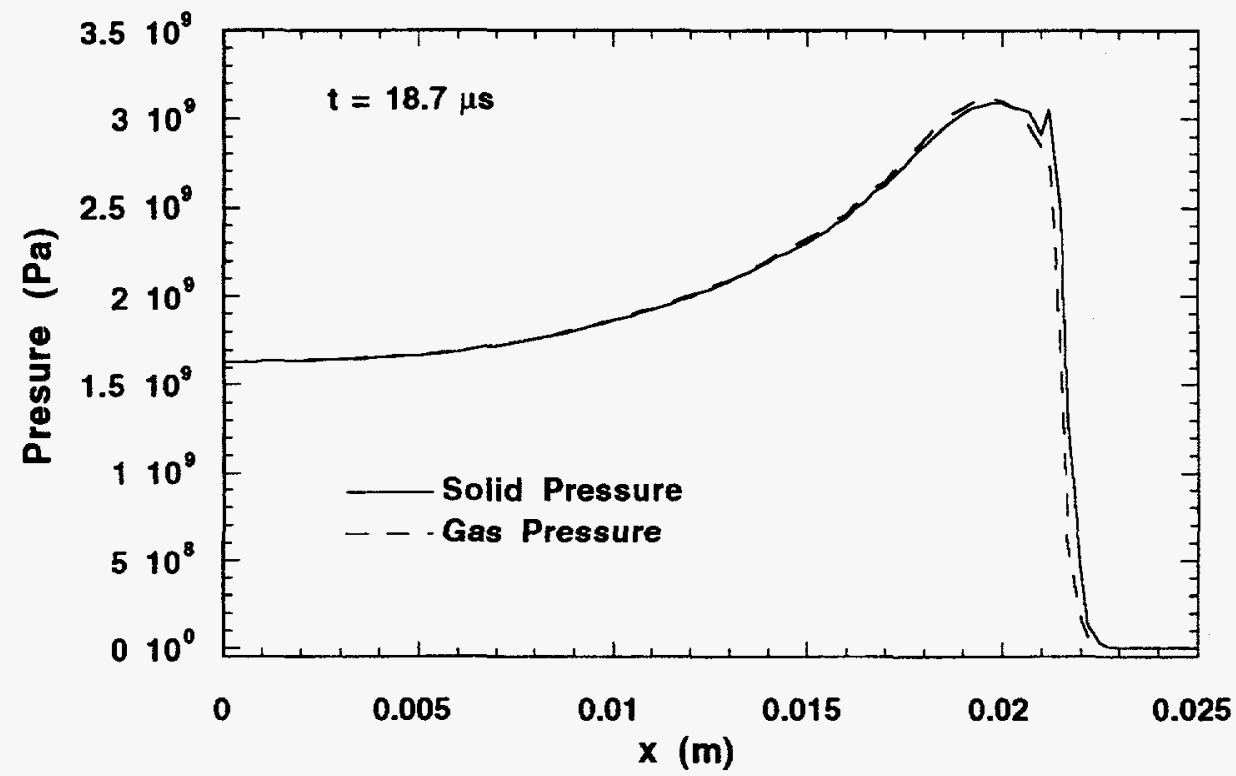

(b)

Fig. 12: A snapshot at $t=10 \mu \mathrm{s}$ (a) and at $t=18.7 \mu \mathrm{s}$ (b) of the solid and gas pressures. The solid pressure rises first. The gas pressure then exceeds the solid pressure once sufficient gas has been generated. 


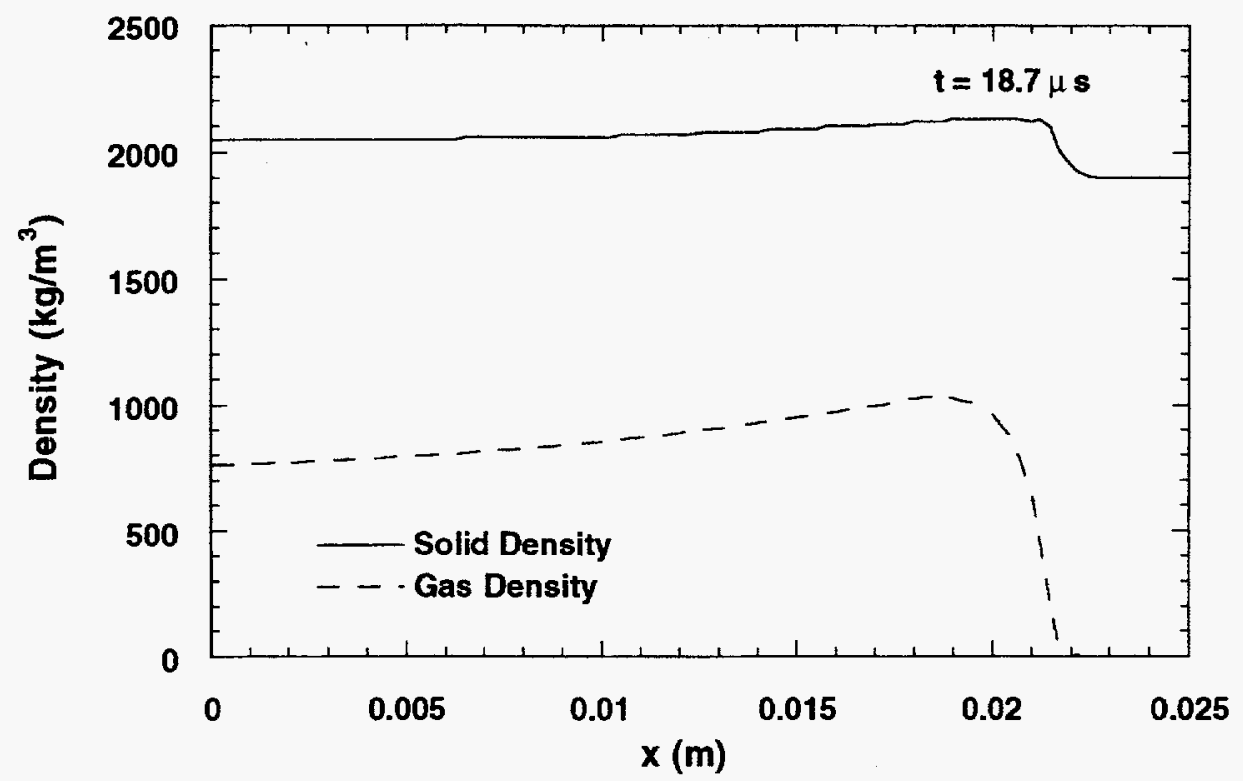

(a)

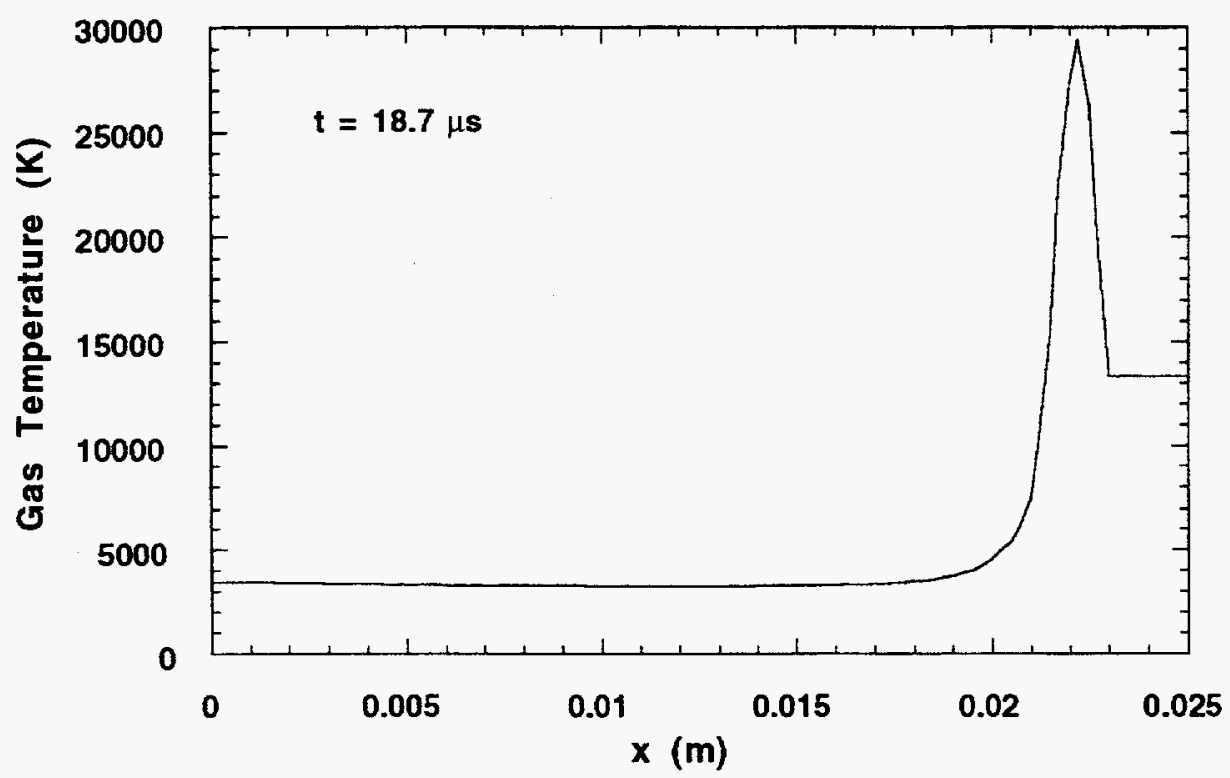

(b)

Fig. 13: A snapshot at $t=18.7 \mu s$ of the solid (upper) and gas (lower) phase densities are displayed in (a). The gas (upper) and solid (lower - nearly coincident with the axis) temperatures are displayed in (b). The densities and temperatures are nowhere equal. 
presented in Figures 10 and 11. The gas particle velocity is above on the initial rise and below in the falling region. Everywhere except at the very toe of these right-going waves, the particle velocities are coincident whenever $\phi_{s} \geq 0.8$. The gas leads slightly in the initial rise. When the reaction produces substantial gas, the gas velocity drops below that of the solid. This is controlled by the strong dependence of $\delta$ on $\phi_{g}$,

$$
\begin{array}{ll}
\phi_{s}=0.95 ; & \delta=4.1 \times 10^{11} \mathrm{~kg} / \mathrm{m}^{3} / \mathrm{s} \\
\phi_{s}=0.40 ; & \delta=3.5 \times 10^{7} \mathrm{~kg} / \mathrm{m}^{3} / \mathrm{s} .
\end{array}
$$

However, the differing compressibilities (i.e., simple mechanical and acoustical) probably also play a role in a fashion similar to what was described in Figures 4 and 5 and in Appendix B [7]. Generally, the velocities are equal except where the combustion is nearing completion and the drag approaches its limiting value

$$
\lim _{\phi_{s} \rightarrow 0} \delta \rightarrow 5.7 \times 10^{5} \mathrm{~kg} / \mathrm{m}^{3} / \mathrm{s} .
$$

In this limit, the e-folding time for velocity differences (obtained for $\phi_{g} \rho_{g}=1000 \mathrm{~kg} / \mathrm{m}^{3}$ ) and using Eq. (41) is $2 \times 10^{-3} \mathrm{~s}$. Thus when $\phi_{s} \geq 0.7$, the velocities are found to be nearly equal, except near shocks.

The solid and gas pressures are compared at $t=10 \mu s$ and $t=18.7 \mu s$ in (a) and (b), respectively, of Figure 12. The solid pressure is above the gas pressure on the initial rise; this is compatible with the compaction process given by Eq. (14). The gas pressure then exceeds the solid pressure. In the compaction wave, the solid and gas pressure need to be different to drive the compaction process. This is consistent with the notion that initially the gas density is very low. Once the reaction starts, the gas pressure rises quickly, leading to the establishment of pressure equilibrium. Thus except in the compaction wave, where

$$
P_{s}-\beta_{s}\left(\phi_{s}\right)-P_{g} \geq 0
$$

the solid and gas pressure are essentially equal.

The solid and gas phase densities and temperatures at $t=18.7 \mu \mathrm{s}$ are shown in Figure 13. The significant observation is that the solid and gas densities and temperatures are very different. A verification run was done using twice the number of computational nodes (i.e., nodes $=200$ ). The results remained essentially unchanged.

In summary, we find that the unadorned BN model has the following properties:

- the permeation of hot combustion gas into partially compacted solid does not occur, and any permeation of gas ahead of the leading solid wave occurs on a scale below the numerical resolution,

- the solid and gas particle velocities are nearly equal for $\phi_{s} \geq 0.8$,

- the solid and gas pressures are nearly equal everywhere except in the compaction wave structure, and 
- the solid and gas densities and temperatures are nowhere equal.

The results on this special, but not atypical, problem suggest that the full richness of a complete two-phase, two-velocity, continuum mixture model (the 7-equation BN model) has not been utilized in generating the above numerical solution. For example, in regions where $\delta$ is very large, the numerical solution shows equal velocities. Any differences in velocity that are seen in such regions are probably spurious since the thin layers associated with large $\delta$ are not being resolved.

Previous work has not recognized the implications of these large drag coefficients. Majda, Hunter and Embid [17] proposed a detonation transition mechanism which requires $V_{s}=V_{g} \pm \mathcal{C}_{g}$. This wave resonance theory for hotspots is based on a singularity observed in the characteristic equations for the $\mathrm{BN}$ model. However, since $V_{s} \approx V_{g}$, this is probably not a generic mechanism for detonation transition.

The compaction work term (i.e., Eq. (25a)) is responsible for the very high gas temperatures at the leading wave. This behavior is nonsensical and should not be included in the model. Equation (25b), that corresponds to all the compaction work remaining in the solid, could be used instead. Clearly, the simplifications in the model that result from assuming nearly equal velocities and nearly equal pressures need to be explored. These reduced models would have simpler wave structures, single velocity advection, plus a smaller system of PDEs.

With this goal in mind, in the next two sections we explore simplified models. We study the one-phase, one-velocity SPA model introduced by Stewart et al. in Section 3 [25]. The two-phase, one-velocity BKS model is then described and studied in Section 4 [26].

The BN model described here is an adaptation of the not very well documented full BN model. The elements needed to get the full model are presented in Appendix A [15]. Three additional reactions (two additional equations) must be added to Eqs. (1)-(22) to get Baer's complete model. These equations control the ignition switch eti in Eq. (10). This area of the modeling is exceedingly weak; much work needs to be done.

\section{The Stewart-Prasad-Asay (SPA) Model}

The SPA model of DDT [25] embraces a different modeling philosophy than that used by Baer and Nunziato. It is built on the principle of "maximum simplicity"; (1) select a DDTlike experiment, model it in the simplest way possible; then (2) select another experiment and modify and add to the model until it agrees with both experiments, etc. Thus the basic structure of the model is continually evolving. Basically, this approach builds the model in a step-by-step process that uses as input only the experiments in question. The $\mathrm{BN}$ approach is built on the philosophy that one begins with the largest possible data set on DDT, safety issues, and detonation/combustion. One then builds a basic modeling structure that accommodates the variables that appear to be most important.

By way of example, temperature is not introduced as a basic SPA modeling variable because temperature is not a part of the DDT-tube test $(x, t)$ - diagram that was the data set to be modeled [27]. BN on the other hand, includes two temperatures (currently three temperatures) because "hotspots" or energy localization is known to be important in the 
combustion of condensed phase, heterogeneous, energetic materials. Both approaches have advantages and disadvantages. The SPA approach examines each issue on its own merits and thus is not subject to the trap of adopting views that are widely held in the community but incorrect. The BN approach attempts to embrace the total data base (some of which is incorrect) and as a result can make more rapid modeling progress when properly guided. The mid ground (the approach we prefer) is to build on what is known, but with a good measure of skepticism and reexamination. With this introduction and having just completed the discussion of the BN model, we now describe the SPA model and the data set to be modeled.

The basic data set to be modeled consists of the distance, time $(x, t)$ data shown in (b) of Figure 14. The data used to get the wave and particle loci displayed came from mechanical and electrical pin switches and optical data from light fibers. Superimposed on these data is the location of a high-density, inert plug that forms during the experiment and that was determined from $\mathrm{x}$-ray measurements. The experiment to be modeled is the DDT of $\phi_{s}{ }^{\circ}=0.73$ granular HMX confined in a thick-walled steel tube and driven by a $160 \mathrm{~m} / \mathrm{s}$ piston. A schematic describing the DDT in such an experiment is shown in (a) of Figure 14. The basic steps in the process are as follows:

- The piston supports a $424 \mathrm{~m} / \mathrm{s}$ compaction wave that raises $\phi_{s}$ to 0.90 .

- After an ignition delay, vigorous combustion begins near the piston and moves through the $\phi_{s}=0.9$ granular HMX. This leads to an increase in pressure ahead of this subsonic reaction front.

- As a result of the increasing pressure, a region upstream of the combustion is compacted to full density and does not react. This region is called the plug.

- The pressure continues to increase, both in the plug and ahead of it, and the plug region continues to grow.

- A detonation is born either at the upper surface of the plug or ahead of it, overruns the compaction wave and consumes all of the remaining HMX in the tube.

- The detonation leaves behind unburnt explosive in the plug that was formed during the process.

The initial goal for the SPA modelers was to build a model that reproduced this $(x, t)$ scenario. We now give a brief description of the SPA model.

In the SPA model, both the solid, unreacted HMX and gaseous, reacted HMX are assumed to have the same $e(P, \rho)$ equation of state. The only difference between the materials is that they have different reference energies (called energy zeros)

$$
E=e(P, \rho)-Q_{\phi}(1-\phi)-Q_{\lambda} \lambda
$$

where $E$ is the specific internal energy function. In Eq. (53) $Q_{\phi} \geq 0$ measures the change in the specific internal energy on compaction, $\phi$ is a volume fraction variable that behaves like 

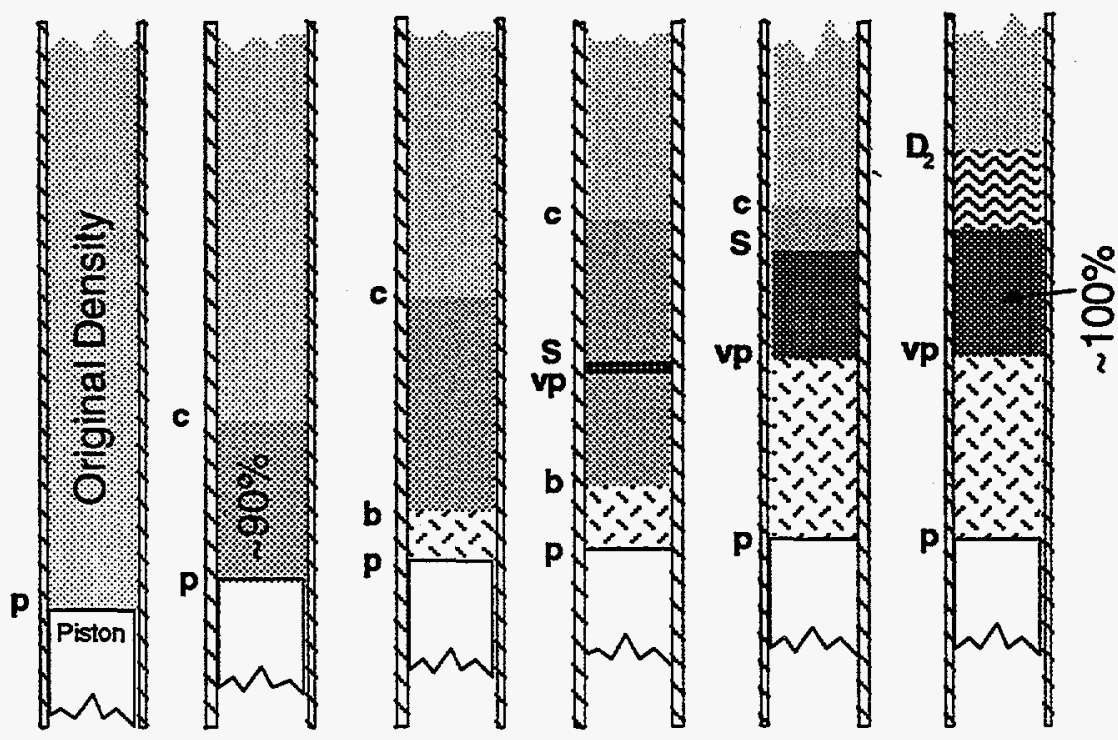

(a)

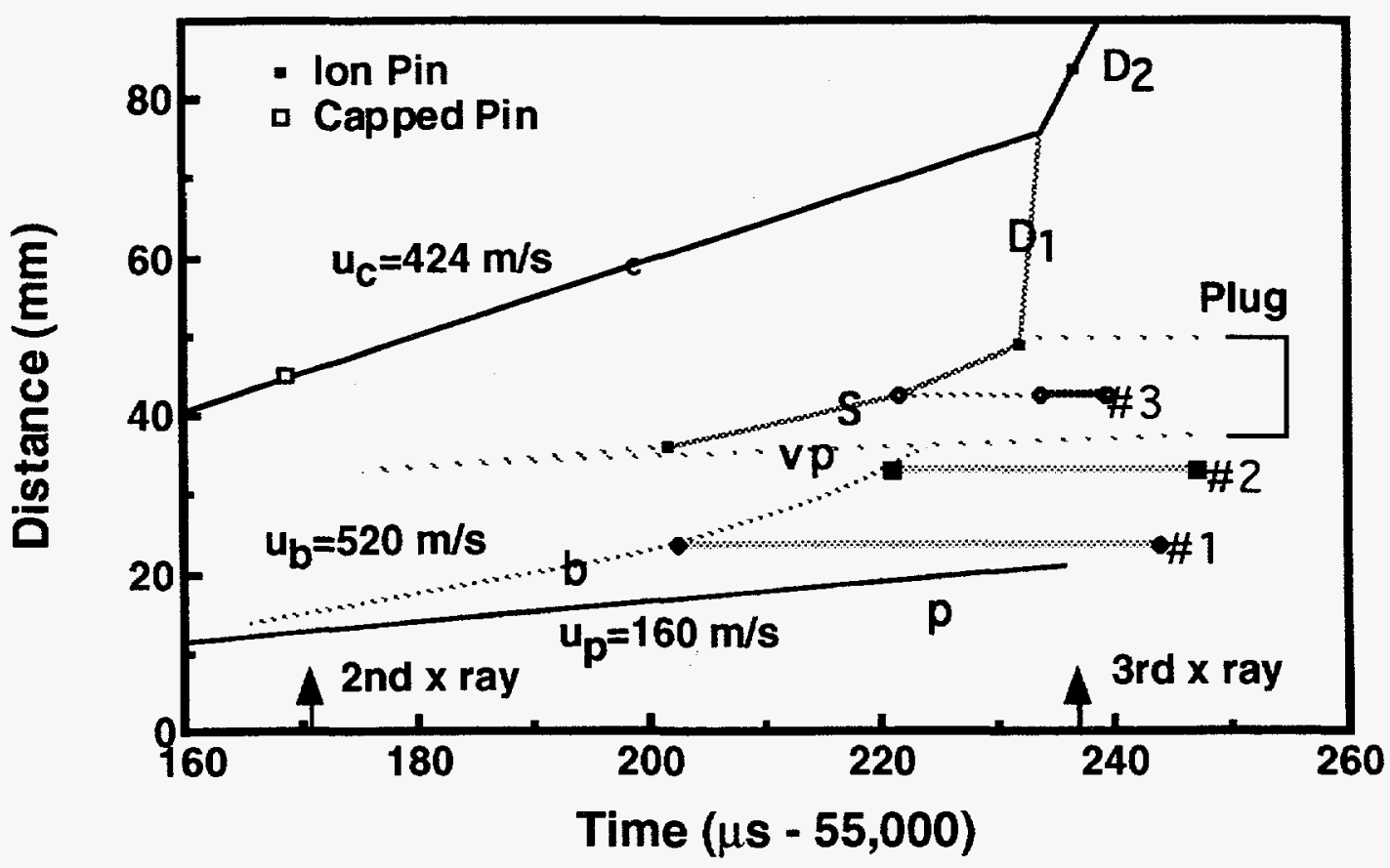

(b)

Fig. 14: Shown above in (a) is a schematic of the DDT process observed in the DDT-tube test. The $(x, t)$ - diagram of the waves and fronts observed in the DDT experiment LANLM8-E5586 (along with x-ray density data) are shown in (b). 


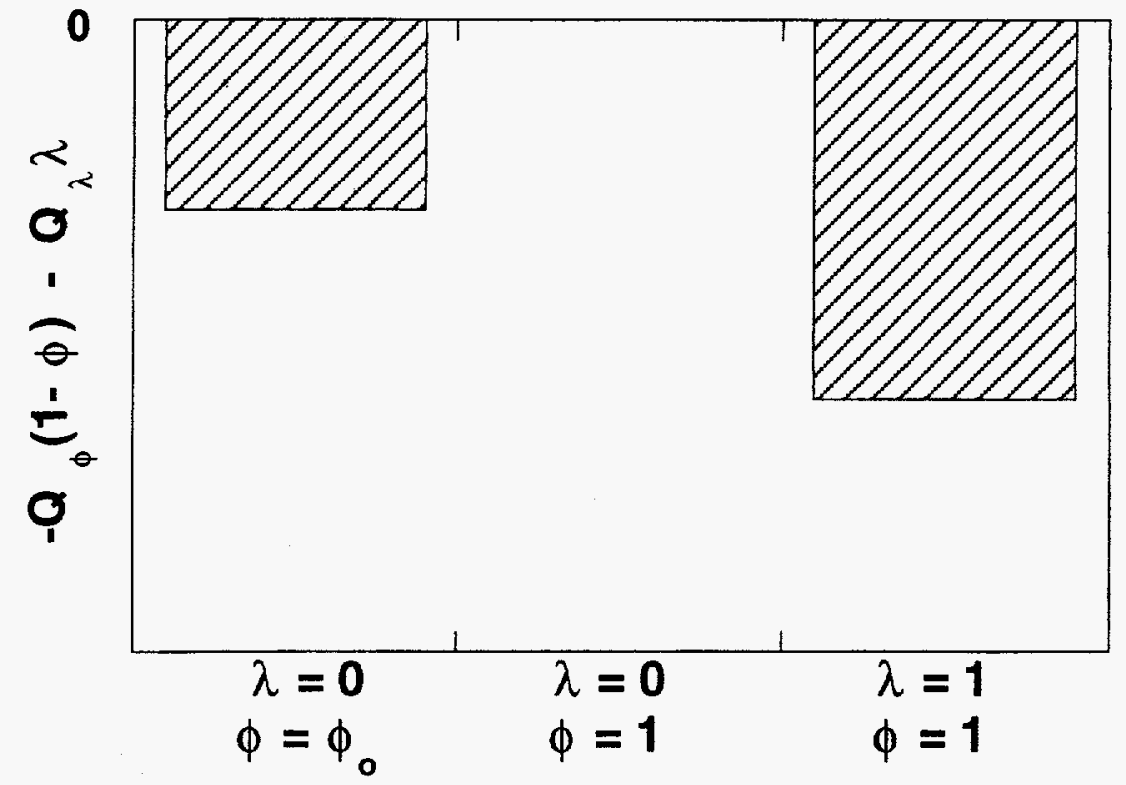

Fig. 15: The heart of the SPA model; the energy budget. The initial energy corresponds to the bottom of the left-most rectangle. Compaction $(\phi=1)$ increases the internal energy to zero (the reference level for the solid). Subsequent reaction reduces the energy to a level corresponding to the bottom of the right-most rectangle. To have a self-sustaining wave, the energy liberated by the reaction must exceed that consumed by compaction.

$\phi_{s}$ of Eq. (7) during pure nonreactive compaction, $Q_{\lambda} \geq 0$ is the change in specific energy on reaction, and $\lambda$ is a mass-fraction like variable that describes the "extent of reaction" ( $\lambda=0$ indicates unreacted and $\lambda=1$ is fully reacted). The change in the $\phi$ and $\lambda$ dependent part of $e$

$$
-Q_{\phi}(1-\phi)-Q_{\lambda} \lambda
$$

is shown as a function of time in Figure 15. Initially, the energy of the material is at the level corresponding to the bottom of the left-most shaded rectangle. On compaction, the energy increases to the zero line (this is an endothermic process, where energy is added to the solid). On completion of reaction, the energy is at the level corresponding to the bottom of the right-most rectangle (this is an exothermic process, where the chemical energy is released). The energetics described by Eq. (54) is the heart of the SPA model.

The time line for the problem is set by the rate law for $\phi$ evolution

$$
R_{\phi}=k_{\phi}(A P-\phi) \geq 0
$$

when $(A P-\phi)>0$ and $\phi<1$, and otherwise $R_{\phi}=0$, and the rate law for $\lambda$ evolution

$$
R_{\lambda}=\left[k_{1} \exp \left(-E_{1}^{\dagger} \rho / P\right)+k_{2} \exp \left(-E_{2}^{\dagger} \rho / P\right)\right] \times(1-\lambda) \geq 0,
$$


when $0 \leq \lambda<1$. In the above, $A$ is the constant in the assumed form of the compaction law

$$
\beta_{s}\left(\phi_{s}\right)=\phi_{s} / A
$$

where $k_{\phi}, k_{1}$, and $k_{2}$ are rate constants and $E_{1}^{\dagger}$ and $E_{2}^{\dagger}$ are activation energies. The overall energy-release rate, defined by

$$
\sigma \cdot r \equiv Q_{\phi} R_{\phi}-Q_{\lambda} R_{\lambda}
$$

contains both endothermic $\left(Q_{\phi} R_{\phi}\right)$ and exothermic $\left(-Q_{\lambda} R_{\lambda}\right)$ contribution. The interplay between these two processes is what drives SPA. To have interesting dynamics, Eq. (58) needs to be positive in some regions and negative in others. The dynamics of this system is analogous to the two-reaction detonation model (irreversible chemistry) described by Fickett and Davis [18]; whence comes the $\sigma \cdot r$ notation in Eq. (58).

Equations (53), (55), (56) and (57) contain the description of the SPA material model. Requiring conservation of mass, momentum and energy

$$
\begin{gathered}
(\rho)_{t}+(V \rho)_{x}=0 \\
(V \rho)_{t}+\left(V^{2} \rho+P\right)_{x}=0,
\end{gathered}
$$

and

$$
\left[\rho\left(E+V^{2} / 2\right)\right]_{t}+\left[\rho V\left(E+P / \rho+V^{2} / 2\right)\right]_{x}=0
$$

where $V$ is the single particle velocity in this one-phase, one-velocity model, and the evolution variables for the internal state variables $\phi$ and $\lambda$

$$
(\rho \phi)_{t}+(V \rho \phi)_{x}=\rho R_{\phi}
$$

and

$$
(\rho \lambda)_{t}+(V \rho \lambda)_{x}=\rho R_{\lambda}
$$

completes the description of the model.

This model has been calibrated to reproduce the $(x, t)$ - diagram shown in Figure 14 for the special case of a polytropic fluid EOS

$$
e(P, \rho)=\frac{P / \rho}{(\gamma-1)}, \quad C^{2}=\frac{\gamma P}{\rho},
$$

where $\gamma$ is the polytropic exponent. The required values of the constants can be found in [25]. In Figure 16, a comparison of the model calculation (b) and the experimental $(x, t)-$ diagram (a) can be found. Generally, most features of the $(x, t)$ - diagram are reproduced. Some questions remain about the plug region (i.e., the region bounded by lines $\mathbf{r}$ and $\mathbf{s}$ in (a) of Figure 16) and the point of onset of detonation. The experiments show that detonation begins at the top side of the plug, and that the plug (the plug is a cold, high-density region) persists after the detonation is $30 \mathrm{~mm}$ past the front side of the plug. The calculations show that onset of detonation is on the bottom of the plug and that the reaction consumes the plug. This result is similar to that observed in the full BN model. Some preliminary work on the formation of an inert plug (onset of detonation on the top of the plug) has 


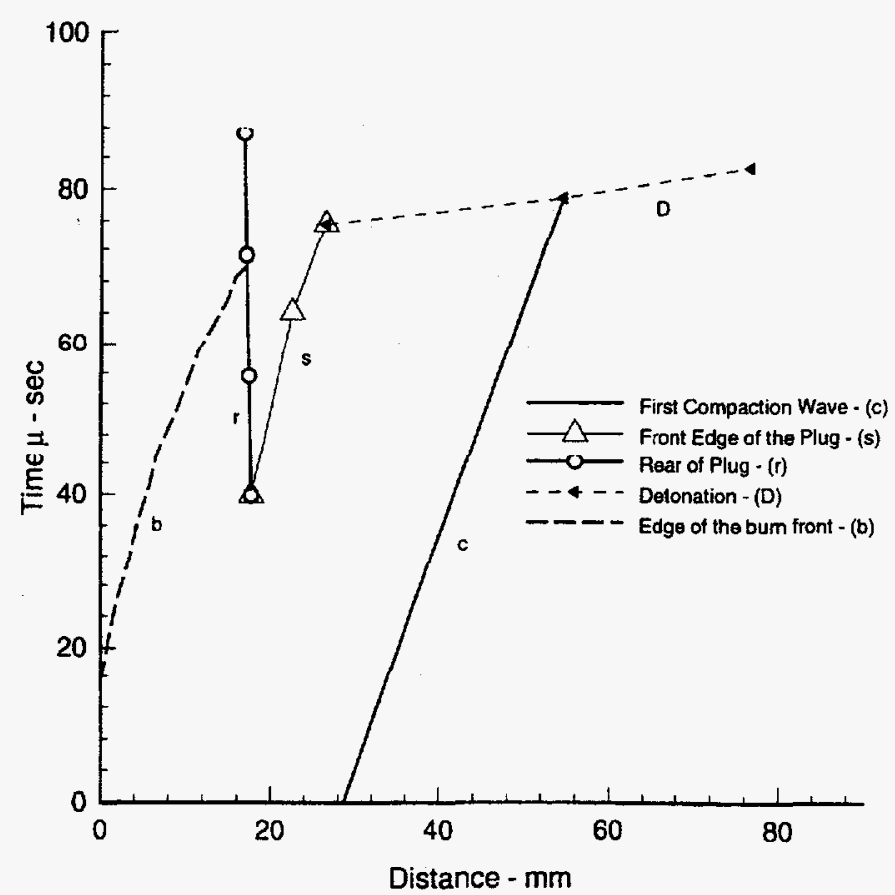

(a)

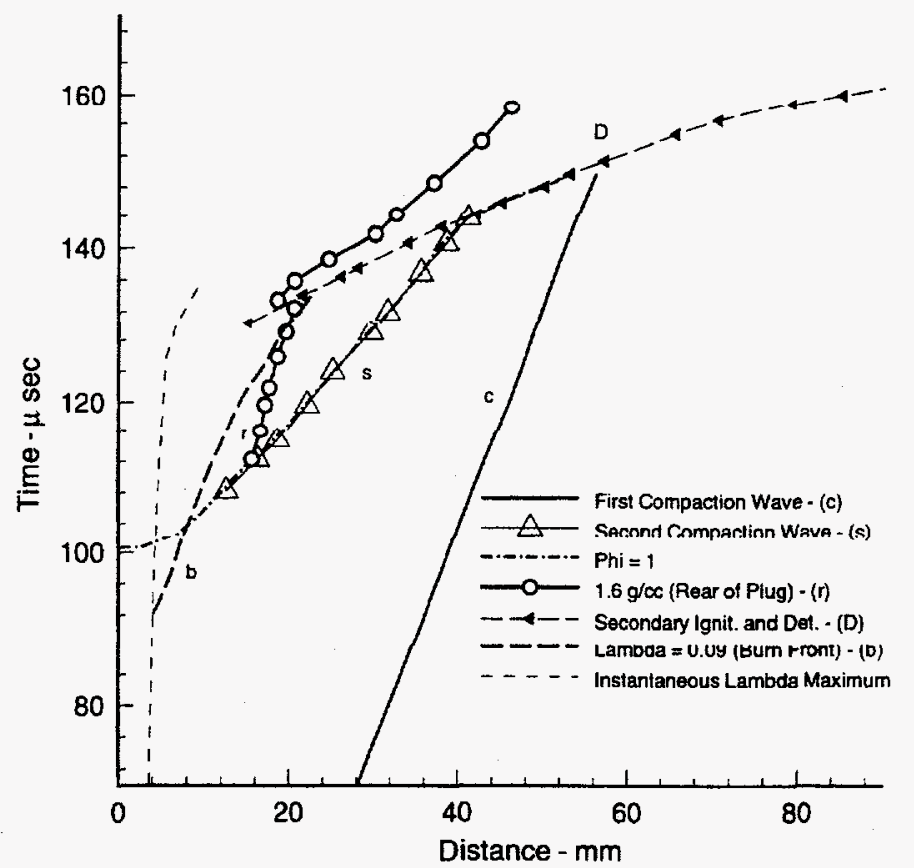

(b)

Fig. 16: The experimental (a) and calculated (b) $(x, t)$ - diagram for experiment LANLM8-E5586. Most experimental features are duplicated. Some questions remain about the lifetime of the plug region (i.e., the upper boundary of the region bounded by lines $\mathbf{r}$ and s). This figure was reprinted from [25] with permission from the author and the American Institute of Physics. 
recently been reported in [28]. The SPA model has yet to be compared to the pressure profiles measured in the DDT-tube, and has not been used to model the shock-initiation like experiments reported in Appendix A.

The hydrodynamics of SPA (a one-phase, one-velocity model) is much simpler than that of the $\mathrm{BN}$ model; that is, it has only one wave family rather than two. However, the onephase aspect of SPA is a serious shortcoming. Equation of state data for condensed phase energetic materials is only available for pure solid, unreacted material and gaseous, reaction products. The EOS response of these materials is quite different; for example, as $P_{s} \rightarrow 0$ then $\rho_{s} \rightarrow \rho_{s}^{\circ}$ whereas $P_{g} \rightarrow 0$ then $\rho_{g}^{o} \rightarrow 0$. This difference in material response needs to be accounted for if for no other reason than these are the only data we have on these materials. Thus, the problems we deal with are inherently two-phase, with EOS behavior such as that given by Eqs. (19)-(22). This automatically increases the number of dependent variables in the problem by two; say two temperatures and pressures instead of one each. In the past, this increase in the number of variables has been handled by introducing two, simple algebraic closure conditions to supplement Eqs. (59)-(61). The most commonly used closures are

- temperature and pressure equilibrium between the phases (i.e., $T=T_{s}=T_{g}$ and $P=P_{s}=P_{g}[2]$, and

- temperature and density equilibrium between the phases (i.e., $T=T_{s}=T_{g}$ and $\rho=\rho_{s}=\rho_{g}[29]$.

The first of these is tantamount to assuming that the solid and gas are mixed on the molecular level, while the second is simply a closure of convenience. Both of these are used in the modeling of prompt shock-initiation of condensed phase, heterogeneous explosives. Which form was used for shock-initiation modeling did not much matter, since the modeling mostly sought to compute distance-to-detonation in regions where many experimental data points were available; the calculations were used to interpolate between data points. A rate law could be found using either closure method that was adequate to predict the distanceto-detonation. It should be noted that the rate law and EOS needed to be treated as an inseparable pair.

For the explosives safety problems that now concern us, we need to be able to predict how damaged materials respond to a much broader range of stimuli than are seen in shock initiation. We cannot possibly calibrate the models to all the situations that we could foresee. Instead, we need to have "better physics" in the models to carry us between fewer, more widely separated data points in the richer parameter space of these problems. As we showed in Section 2, the algebraic closures described above do not constitute a good physical representation of the problem. In the next section, we show that the richer structure of the BN model can be maintained in a framework that is suitable for implementation into currently available one-velocity family of multidimensional, multimaterial hydrodynamics codes, such as Mesa3D [30]. What we describe next is a formal asymptotic reduction of Eqs. (1)-(8) in the limit that $\left(V_{s}-V_{g}\right) \rightarrow 0$. 


\section{The Bdzil-Kapila-Stewart (BKS) Model}

The idea of taking formal asymptotic limits on the BN model is attributable to Kapila [31]. We describe that work from a slightly different point of view than Kapila's and include some additional results.

The BN model contains two temperatures, pressures, densities and velocity fields; one each for the solid and the gas. The interaction terms given by Eqs. (10), (12), (14) and (15) describe how these fields communicate with one another. For example, the drag interaction coefficient $\delta$ says how momentum is transferred between the phases, whereas the heat-transfer coefficient $\mathcal{H}$ says how energy is transferred between the phases in response to interphase temperature differences. Although one could argue about the details of the modeling structure in the BN model (particularly about the form of the exchange terms), this methodology has proven useful in many areas of technology. In modeling DDT, we need to be able to treat the burning of the solid, which is thought to take place in the gas phase, and detonation of heterogeneous explosives, for which we know that energy localization and "hotspots" are important. Thus, it is quite natural to think about the composite material as being made up of different "phases" whose thermodynamic variables have different values. The hydrodynamics of a two-phase system is much richer (also harder to understand) than that of a one-phase, one-velocity system. However, under appropriate limits, two-phase models reduce to simpler one-phase ones.

What we decided to do is assume that the BN model contains a complete description of the physics, and study what reductions could be affected by taking rigorous asymptotic limits based on special values for the phase interaction parameters. The limits of large drag $\delta \rightarrow \infty$ and high heat-transfer rates $k_{g} \rightarrow \infty$ were considered. This was in keeping with a remark made by Don Drew [32] that one should always examine multiphase physics at one level of magnification higher than the level treated in one's global modeling. What we elected to do is treat the two-phase, two-velocity model as the subscale model. Then by taking limits, see how one-phase, one-velocity models could be derived from it. Here we consider only one limit; the limit of large drag, $\delta \rightarrow \infty$. The development presented here is an adaptation of Kapila's original work and includes both modifications and extensions.

\subsection{The Large Drag Limit}

We first rewrite the BN equations (Eqs. (1)-(6)) in a form that highlights the velocity difference, since in the limit of large $\delta$ one expects the velocity difference to be small. To do this, we define some auxiliary variables related to the two-phase mixture

$$
\frac{\frac{\text { mixture density }}{\text { mass-weighted velocity }}}{\text { mixture pressure }}
$$

$$
\begin{aligned}
& \langle\rho\rangle=\phi_{s} \rho_{s}+\phi_{g} \rho_{g}, \\
& V=\left(\phi_{s} \rho_{s} V_{s}+\phi_{g} \rho_{g} V_{g}\right) /\langle\rho\rangle \\
& \langle P\rangle=\phi_{s} P_{s}+\phi_{g} P_{g} \\
& e=\left(\phi_{s} \rho_{s} e_{s}+\phi_{g} \rho_{g} e_{g}\right) /\langle\rho\rangle
\end{aligned}
$$

where $\langle\rho\rangle$ and $\langle P\rangle$ are the mixture density and pressure, respectively, and $V$ and $e$ are the mass-weighted or baracentric velocity and specific internal energy, respectively. The 
following relations will also be used

$$
V_{g}=V-\left(V_{s}-V_{g}\right) \phi_{s} \rho_{s} /\langle\rho\rangle
$$

and

$$
V_{s}=V+\left(V_{s}-V_{g}\right) \phi_{g} \rho_{g} /\langle\rho\rangle
$$

to transform to $V$ and $\left(V_{s}-V_{g}\right)$ as variables.

The first set of equations we write down are the conservation equations for the overall mixture, obtained by adding Eqs. (1)-(2), adding Eqs. (3)-(4) and adding Eqs. (5)-(6)

$$
\begin{gathered}
(\langle\rho\rangle)_{t}+(\langle\rho\rangle V)_{x}=0 \\
(\langle\rho\rangle V)_{t}+\left[\langle P\rangle+\langle\rho\rangle V^{2}+\left(V_{s}-V_{g}\right)^{2}\left(\phi_{s} \rho_{s}\right)\left(\phi_{g} \rho_{g}\right) /\langle\rho\rangle\right]_{x}=0
\end{gathered}
$$

and

$$
\begin{aligned}
\left\{\langle \rho \rangle \left[e+V^{2} / 2\right.\right. & \left.\left.+\left(V_{s}-V_{g}\right)^{2}\left(\phi_{s} \rho_{s}\right)\left(\phi_{g} \rho_{g}\right) / 2 /\langle\rho\rangle^{2}\right]\right\}_{t} \\
+\{\langle\rho\rangle V(e & \left.+\langle P\rangle /\langle\rho\rangle+V^{2} / 2\right) \\
& +\left(V_{s}-V_{g}\right)\left(e_{s}-e_{g}+P_{s} / \rho_{s}-P_{g} / \rho_{g}\right)\left(\phi_{s} \rho_{s}\right)\left(\phi_{g} \rho_{g}\right) /\langle\rho\rangle \\
& +3 V\left(V_{s}-V_{g}\right)^{2}\left(\phi_{s} \rho_{s}\right)\left(\phi_{g} \rho_{g}\right) / 2 /\langle\rho\rangle \\
& \left.+\left(V_{s}-V_{g}\right)^{3}\left(\phi_{g} \rho_{g}-\phi_{s} \rho_{s}\right)\left(\phi_{s} \rho_{s}\right)\left(\phi_{g} \rho_{g}\right) / 2 /\langle\rho\rangle^{2}\right\}_{x}=0 .
\end{aligned}
$$

When $\left(V_{s}-V_{g}\right) \rightarrow 0$, these are simply the two-phase mixture equations that are solved in a code like Mesa3D. They have the same form as the one-phase equations given by Eqs. (59)-(61).

Next we substitute Eqs. (69)-(70) into the two BN mass and energy equations so as to highlight $\left(V_{s}-V_{g}\right)$. In doing this, we rewrite the two energy equations so as to focus on the internal rather than the total energy. Doing this, we get

$$
\begin{gathered}
\left(\phi_{s} \rho_{s}\right)_{t}+\left[\phi_{s} \rho_{s} V+\left(V_{s}-V_{g}\right)\left(\phi_{s} \rho_{s}\right)\left(\phi_{g} \rho_{g}\right) /\langle\rho\rangle\right]_{x}=C_{s}^{\dagger} \\
\left(\phi_{g} \rho_{g}\right)_{t}+\left[\phi_{g} \rho_{g} V-\left(V_{s}-V_{g}\right)\left(\phi_{s} \rho_{s}\right)\left(\phi_{g} \rho_{g}\right) /\langle\rho\rangle\right]_{x}=-C_{s}^{\dagger}
\end{gathered}
$$

and

$$
\begin{aligned}
\frac{D_{s} e_{s}}{D t}-\frac{P_{s}}{\rho_{s}^{2}} \frac{D_{s} \rho_{s}}{D t} & =\left\{\frac{\beta_{s}\left(\phi_{s}\right) \mathcal{F}}{\phi_{s} \rho_{s}}-\frac{\mathcal{H}\left(T_{s}-T_{g}\right)}{\phi_{s} \rho_{s}}\right\} \text { or }\left\{\frac{\left(P_{s}-P_{g}\right) \mathcal{F}}{\phi_{s} \rho_{s}}-\frac{\mathcal{H}\left(T_{s}-T_{g}\right)}{\phi_{s} \rho_{s}}\right\}, \\
\frac{D_{g} e_{g}}{D t}-\frac{P_{g}}{\rho_{g}^{2}} \frac{D_{g} \rho_{g}}{D t} & =\frac{\delta\left(V_{s}-V_{g}\right)^{2}}{\phi_{g} \rho_{g}}+\left[e_{g}-e_{s}+P_{g}\left(\frac{1}{\rho_{g}}-\frac{1}{\rho_{s}}\right)\right] \frac{C_{s}^{\dagger}}{\phi_{g} \rho_{g}} \\
& +\left\{\frac{\left(P_{s}-\beta_{s}\left(\phi_{s}\right)-P_{g}\right) \mathcal{F}}{\phi_{g} \rho_{g}}+\frac{\mathcal{H}\left(T_{s}-T_{g}\right)}{\phi_{g} \rho_{g}}\right\} \text { or }\left\{\frac{\mathcal{H}\left(T_{s}-T_{g}\right)}{\phi_{g} \rho_{g}}\right\},
\end{aligned}
$$

where

$$
\frac{D_{i}}{D t}=\frac{\partial}{\partial t}+V_{i} \frac{\partial}{\partial x}
$$


with $\mathrm{i}=\mathrm{s}, \mathrm{g}$ and where $V_{i}$ is given by Eqs. (69)-(70). As before, the a,b designation in Eqs. $(76 \mathrm{a}, \mathrm{b})-(77 \mathrm{a}, \mathrm{b})$ denotes whether $\mathrm{Eq} .(25 \mathrm{a})$ or Eq. (25b) is used to represent compaction work (recall Eq. (25b) corresponds to all the compaction work going to the solid). Equation (25a) corresponds to the BN assumption, while we prefer Eq. (25b). To the above four equations (Eqs. (74)-(77)) two more need to be added to get a set equivalent to the six BN equations. As the remaining two equations we take the mixture momentum equation, Eq. (72) and a derived equation for the velocity difference

$$
\begin{aligned}
&\left(V_{s}-V_{g}\right)_{t}+ {\left[V\left(V_{s}-V_{g}\right)+\left(V_{s}-V_{g}\right)^{2}\left(\phi_{g} \rho_{g}-\phi_{s} \rho_{s}\right) / 2 /\langle\rho\rangle\right]_{x}=} \\
&+\left(V_{s}-V_{g}\right) C_{s}^{\dagger}\left(\phi_{s} \rho_{s}-\phi_{g} \rho_{g}\right) / 2 /\left(\phi_{s} \rho_{s}\right)\left(\phi_{g} \rho_{g}\right) \\
&+\langle\rho\rangle\left[P_{g}\left(\phi_{s}\right)_{x}-\delta\left(V_{s}-V_{g}\right)\right] /\left(\phi_{s} \rho_{s}\right) /\left(\phi_{g} \rho_{g}\right) \\
&+\left(\phi_{g} P_{g}\right)_{x} /\left(\phi_{g} \rho_{g}\right)-\left(\phi_{s} P_{s}\right)_{x} /\left(\phi_{s} \rho_{s}\right) .
\end{aligned}
$$

Although Eq. (79) looks complicated, it will be useful in helping to derive the large $\delta$ BKS model. Equation (79) is an evolution equation for the velocity difference. Given that initially $\left(V_{s}-V_{g}\right)=0$, Eq. (79) shows that large gradients in pressure drive $\left(V_{s}-V_{g}\right)$ away from zero, while the drag interaction $\delta\left(V_{s}-V_{g}\right)$ returns $\left(V_{s}-V_{g}\right)$ to zero. Compaction work does not directly influence $\left(V_{s}-V_{g}\right)$. Equations $(72),(74)-(77 \mathrm{a}, \mathrm{b}),(79)$ are equivalent to the original six BN equations (i.e., Eqs. (1)-(6)).

Another equation that is derivable from Eqs. (1)-(6) gives some insights into the flow dynamics of this problem; the equation for $\left(\phi_{s} \rho_{s} / \phi_{g} \rho_{g}\right)$ suggested by Menikoff. We record it here even though no further use is made of it in the derivation of the BKS model

$$
\begin{aligned}
\left(\phi_{s} \rho_{s} / \phi_{g} \rho_{g}\right)_{t} & +V\left(\phi_{s} \rho_{s} / \phi_{g} \rho_{g}\right)_{x}=C_{s}^{\dagger}\langle\rho\rangle /\left(\phi_{g} \rho_{g}\right)^{2} \\
& -\left(V_{s}-V_{g}\right)_{x}\left(\phi_{s} \rho_{s} / \phi_{g} \rho_{g}\right)+\left(V_{s}-V_{g}\right)\left[\langle\rho\rangle /\left(\phi_{s} \rho_{s}\right) /\left(\phi_{g} \rho_{g}\right)\right]_{x}\left(\phi_{s} \rho_{s}\right)^{2} /\langle\rho\rangle .
\end{aligned}
$$

Equation (80) says that when $C_{s}^{\dagger}=0$ and $\left(V_{s}-V_{g}\right)=0$, then $\left(\phi_{s} \rho_{s} / \phi_{g} \rho_{g}\right)$ is constant along a particle path (except for shocks). This could serve as a starting point for deriving an algebraic EOS closure for a SPA-like theory that utilizes separate solid and gas equations of state. Here we don't pursue this issue further. We will now derive the BKS model.

The original large drag limit approximation makes the following assumptions:

- $\delta$ is the large parameter,

- all other rate processes (transformations) are slow by comparison (i.e., the speed of these other rates is $O(1)$ ),

- both the time and space variations of the solution occur on an $O(1)$ scale,

- $\left(V_{s}-V_{g}\right)=O(1 / \delta)$, so that

- discontinuities such as shocks are excluded from consideration. 
With these assumptions, Eq. (79) admits a distinguished limit with

$$
\left(V_{s}-V_{g}\right)=O(1 / \delta)
$$

and under which Eq. (79) reduces to an algebraic relation for the velocity difference

$$
\delta\left(V_{s}-V_{g}\right)=P_{g}\left(\phi_{s}\right)_{x}+\left[\left(\phi_{s} \rho_{s}\right)\left(\phi_{g} P_{g}\right)_{x}-\left(\phi_{g} \rho_{g}\right)\left(\phi_{s} P_{s}\right)_{x}\right] /\langle\rho\rangle
$$

Equation (82) is the reduced form of one of the BN equations, Eq. (79). Using this result and the assumption that $\left(V_{s}-V_{g}\right)=O(1 / \delta)$, causes Eqs. (74)-(75) to reduce to

$$
\left(\phi_{s} \rho_{s}\right)_{t}+\left(\phi_{s} \rho_{s} V\right)_{x}=C_{s}^{\dagger}
$$

and

$$
\left(\phi_{g} \rho_{g}\right)_{t}+\left(\phi_{g} \rho_{g} V\right)_{x}=-C_{s}^{\dagger}
$$

the mixture momentum equation Eq. (72) to reduce to

$$
(\langle\rho\rangle V)_{t}+\left(\langle P\rangle+\langle\rho\rangle V^{2}\right)_{x}=0
$$

and the two-phase, total energy equations (i.e., equations for kinetic plus internal energy, Eqs. (5)-(6)) to reduce to

$$
\begin{aligned}
{\left[\phi_{s} \rho_{s}\left(e_{s}+V^{2} / 2\right)\right]_{t} } & +\left[\phi_{s} \rho_{s} V\left(e_{s}+V^{2} / 2\right)+\phi_{s} P_{s} V\right]_{x}= \\
& +V\left[\left(\phi_{g} \rho_{g}\right)\left(\phi_{s} P_{s}\right)_{x}-\left(\phi_{s} \rho_{s}\right)\left(\phi_{g} P_{g}\right)_{x}\right] /\langle\rho\rangle+\left(e_{s}+V^{2} / 2\right) C_{s}^{\dagger} \\
& -\left\{\left(P_{s}-\beta_{s}\left(\phi_{s}\right)\right) \mathcal{F}+\mathcal{H}\left(T_{s}-T_{g}\right)\right\} \text { or }-\left\{P_{g} \mathcal{F}+\mathcal{H}\left(T_{s}-T_{g}\right)\right\}
\end{aligned}
$$

and

$$
\begin{aligned}
{\left[\phi_{g} \rho_{g}\left(e_{g}+V^{2} / 2\right)\right]_{t} } & +\left[\phi_{g} \rho_{g} V\left(e_{g}+V^{2} / 2\right)+\phi_{g} P_{g} V\right]_{x}= \\
& -V\left[\left(\phi_{g} \rho_{g}\right)\left(\phi_{s} P_{s}\right)_{x}-\left(\phi_{s} \rho_{s}\right)\left(\phi_{g} P_{g}\right)_{x}\right] /\langle\rho\rangle-\left(e_{s}+V^{2} / 2\right) C_{s}^{\dagger} \\
& +\left\{\left(P_{s}-\beta_{s}\left(\phi_{s}\right)\right) \mathcal{F}+\mathcal{H}\left(T_{s}-T_{g}\right)\right\} \text { or } \quad+\left\{P_{g} \mathcal{F}+\mathcal{H}\left(T_{s}-T_{g}\right)\right\}
\end{aligned}
$$

On using Eq. (81) and putting Eq. (7) in conservation form, the porosity evolution equation becomes

$$
\left(\langle\rho\rangle \phi_{s}\right)_{t}+\left(\phi_{s}\langle\rho\rangle V\right)_{x}=\langle\rho\rangle \mathcal{F}+\langle\rho\rangle C_{s}^{\dagger} / \rho_{s} .
$$

Equations (83)-(88) are the BKS equations as they were originally written and as they are coded in "September-BKS." Thus in this limit, the 7-equation BN model has been reduced to a 6-equation model for the variables $V, \phi_{s}, \rho_{s}, \rho_{g}, P_{s}$ and $P_{g}$, where $e_{s}\left(P_{s}, \rho_{s}\right)$ and $e_{g}\left(P_{g}, \rho_{g}\right)$ are known functions Eqs. (19)-(22).

For a hydrodynamic code such as Mesa3D, a better form of these equations is to use the three mixture equations (taking the limit $\left(V_{s}-V_{g}\right)=O(1 / \delta)$ )

$$
\begin{gathered}
(\langle\rho\rangle)_{t}+(\langle\rho\rangle V)_{x}=0 \\
(\langle\rho\rangle V)_{t}+\left[\langle P\rangle+\langle\rho\rangle V^{2}\right]_{x}=0
\end{gathered}
$$

and

$$
\left[\langle\rho\rangle\left(e+V^{2} / 2\right)\right]_{t}+\left[\langle\rho\rangle V\left(e+\langle P\rangle /\langle\rho\rangle+V^{2} / 2\right)\right]_{x}=0
$$


and the two solid phase equations

$$
\left(\phi_{s} \rho_{s}\right)_{t}+\left(\phi_{s} \rho_{s} V\right)_{x}=C_{s}^{\dagger}
$$

and say

$$
\frac{D_{s} e_{s}}{D t}-\frac{P_{s}}{\rho_{s}^{2}} \frac{D_{s} \rho_{s}}{D t}=\left\{\frac{\beta_{s}\left(\phi_{s}\right) \mathcal{F}}{\phi_{s} \rho_{s}}-\frac{\mathcal{H}\left(T_{s}-T_{g}\right)}{\phi_{s} \rho_{s}}\right\} \quad \text { or } \quad\left\{\frac{\left(P_{s}-P_{g}\right) \mathcal{F}}{\phi_{s} \rho_{s}}-\frac{\mathcal{H}\left(T_{s}-T_{g}\right)}{\phi_{s} \rho_{s}}\right\} \text {, }
$$

or

$$
\begin{aligned}
{\left[\phi_{s} \rho_{s}\left(e_{s}+V^{2} / 2\right)\right]_{t} } & +\left[\phi_{s} \rho_{s} V\left(e_{s}+V^{2} / 2\right)+\phi_{s} P_{s} V\right]_{x}= \\
& +V\left[\left(\phi_{g} \rho_{g}\right)\left(\phi_{s} P_{s}\right)_{x}-\left(\phi_{s} \rho_{s}\right)\left(\phi_{g} P_{g}\right)_{x}\right] /\langle\rho\rangle+\left(e_{s}+V^{2} / 2\right) C_{s}^{\dagger} \quad(94 a, b) \\
& -\left\{\left(P_{s}-\beta_{s}\left(\phi_{s}\right)\right) \mathcal{F}+\mathcal{H}\left(T_{s}-T_{g}\right)\right\} \text { or }-\left\{P_{g} \mathcal{F}+\mathcal{H}\left(T_{s}-T_{g}\right)\right\},
\end{aligned}
$$

and the porosity evolution equation

$$
\left(\langle\rho\rangle \phi_{s}\right)_{t}+\left(\phi_{s}\langle\rho\rangle V\right)_{x}=\langle\rho\rangle \mathcal{F}+\langle\rho\rangle C_{s}^{\dagger} / \rho_{s}
$$

Although the derivation of the 6-equation, one-velocity BKS model assumed a shock free flow, our intent is to use the equations in the presence of shock waves. The one disturbing thing about the structure of these equations is that they contain a term that is not in conservation form. That is, the source for Eq. $(94 \mathrm{a}, \mathrm{b})$ contains the term

$$
V\left[\left(\phi_{g} \rho_{g}\right)\left(\phi_{s} P_{s}\right)_{x}-\left(\phi_{s} \rho_{s}\right)\left(\phi_{g} P_{g}\right)_{x}\right] /\langle\rho\rangle,
$$

which can not be integrated across shock waves without providing some additional information. The structure of this term is similar in many respects to the "nozzling" term (Eq. (23a) and (23b) and Eqs. (43) and (44)) discussed in Section 2. Like Eq. (23a), Eq.(96) contains no adjustable parameters, and it can not be integrated across discontinuities without further information. For Eq. (23a), material interfaces were the only problem. For Eq. (96), the discontinuity that presents a problem is the ubiquitous shock.

The nonconservation form issue does not present a serious problem in many situations that we encounter. If the rapid changes in pressure occur in a smooth feature such as a compaction wave, then no problems arise. Similarly, from Eq. (96) it is clear that when either of the phases is in a minority, then Eq. (96) is a small source term and can be neglected. Many of the problems we consider have these features, and thus the form of Eq. (96) does not lead to real difficulties. This nonconservation form issue does raise an important theoretical question: can the shock state be uniquely defined? We can get some clues about this by examining the alternative formulation of the problem contained in Eq. $(93 \mathrm{a}, \mathrm{b})$.

Equation (93a, b) does not contain the nonconservative term displayed in Eq. (96). After some minor algebra, Eq. (93a, b) can be put in a form that is integrable across a shock. Using the definition of the frozen sound speed

$$
\mathcal{C}_{s}^{2}=\left(\frac{P_{s}}{\rho_{s}^{2}}-\left(\frac{\partial e_{s}}{\partial \rho_{s}}\right)_{P_{s}}\right) /\left(\frac{\partial e_{s}}{\partial P_{s}}\right)_{\rho_{s}},
$$


Eq. $(93 a, b)$ can be rewritten as

$$
\frac{D_{s} P_{s}}{D t}-\mathcal{C}_{s}^{2} \frac{D_{s} \rho_{s}}{D t}=\left\{\frac{\beta_{s}\left(\phi_{s}\right) \mathcal{F}}{\phi_{s} \rho_{s}\left(\frac{\partial e_{s}}{\partial P_{s}}\right)_{\rho_{s}}}-\frac{\mathcal{H}\left(T_{s}-T_{g}\right)}{\phi_{s} \rho_{s}\left(\frac{\partial e_{s}}{\partial P_{s}}\right)_{\rho_{s}}}\right\} \quad \text { or } \quad\left\{\frac{\left(P_{s}-P_{g}\right) \mathcal{F}}{\phi_{s} \rho_{s}\left(\frac{\partial e_{s}}{\partial P_{s}}\right)_{\rho_{s}}}-\frac{\mathcal{H}\left(T_{s}-T_{g}\right)}{\phi_{s} \rho_{s}\left(\frac{\partial e_{s}}{\partial P_{s}}\right)_{\rho_{s}}}\right\} \text {. }
$$

For equations of state for which $\mathcal{C}_{s}^{2}$ is of the form

$$
\mathcal{C}_{s}^{2}=\mathcal{M}\left(P_{s}\right) \mathcal{N}\left(\rho_{s}\right)
$$

(e.g., this is true of the polytropic and Tait EOS) Eq. (98a, b) can formally be put in conservation form. By way of example, for a polytropic EOS

$$
\mathcal{C}_{s}^{2}=\frac{\gamma_{s} P_{s}}{\rho_{s}}
$$

with $\gamma_{s}$ the polytropic exponent for this solid EOS, we find that

$$
(\langle\rho\rangle \Xi)_{t}+(\langle\rho\rangle \Xi V)_{x}=\left(\frac{\left(\gamma_{s}-1\right)\langle\rho\rangle}{\phi_{s} P_{s}}\right)\left\{\beta_{s}\left(\phi_{s}\right) \mathcal{F}-\mathcal{H}\left(T_{s}-T_{g}\right)\right\} \text { or }\left\{\left(P_{s}-P_{g}\right) \mathcal{F}-\mathcal{H}\left(T_{s}-T_{g}\right)\right\}
$$

where

$$
\Xi=\ln \left(P_{s} / \rho_{s}^{\gamma_{s}}\right)
$$

Focusing attention on a simple noncompacting shock, for which both $\mathcal{F}=0$ and $\mathcal{K}=0$, it follows that $\mathrm{Eq}$. (101a, b) can be integrated to get

$$
\frac{P_{s}}{\rho_{s} \gamma_{s}}=f_{s}\left(S_{s}\right)
$$

where $f_{s}\left(S_{s}\right)$ is a known function of the solid entropy, $S_{s}$ generated at the shock. The calculation in Appendix B shows that the value of this function can be found, and the shock state (both gas and solid) can be uniquely determined. The value of $S_{s}$ is determined completely by the pure solid Hugoniot jump conditions, and thus can not be determined without the aid of the solid momentum equation (recall that BKS has only a mixture momentum equation). Thus, in a sense BKS is incomplete. Since the change in solid entropy across typical shocks is small, the error made in assuming that the solid is isentropic is probably small. Using Eq. (18) to compute the change in solid entropy, $\Delta S_{s}$, across the shock, we find

$$
\Delta S_{s}=C_{v s}\left[\ln \left(\frac{T_{s}}{T_{s}{ }^{\circ}}\right)-\frac{g o v}{\rho_{s}{ }^{\circ}}\left(1-\rho_{s}^{o} / \rho_{s}\right)\right]=O\left(\left(1-\rho_{s}^{o} / \rho_{s}\right)^{3}\right),
$$

so that the jump in solid entropy across a shock is $O\left(\left(1-\rho_{s}{ }^{o} / \rho_{s}\right)^{3}\right)$. Part (a) of Figure 13 shows that $\rho_{s}{ }^{\circ} / \rho_{s}$ is nearly one across a typical shock. For such situations, the alternate "conservative" formulation of BKS using Eq. (93a, b) should give good results. We will show that this is the case by considering some examples. In order to treat the general case, a way needs to be found that captures the proper entropy across shocks. One method is to "regularize" or smooth the solution by adding dissipation such as viscosity. For example, regularizing the Burgers equation with a viscous term gives the proper conservation law from the infinity of possible conservation laws for that equation. Another way is to use the velocity difference equation to "smear out" the BKS shock, thereby capturing the correct solid entropy. We will discuss this later approach in the following section. 


\subsection{Solutions of the BKS Model}

The "September-BKS" code solves Eqs. (83)-(88), with $\phi_{g}+\phi_{s}=1$ and $\mathcal{F}, C_{s}^{\dagger}, \beta_{s}\left(\phi_{s}\right), \mathcal{H}$, $T_{s}\left(e_{s}, \rho_{s}\right), P_{s}\left(e_{s}, \rho_{s}\right), T_{g}\left(e_{g}, \rho_{g}\right)$ and $P_{g}\left(e_{g}, \rho_{g}\right)$ given by Eqs. (9), (10), (13), (15), and (19)(22), respectively [19]. A description of the numerical algorithm is found at the beginning of Section 2.2 in the text surrounding Eqs. (33) and (34). The boundary conditions and material constants are entered via a Fortran namelist in the file "input." The parameter values are returned in the file "septout," for which an example is displayed in Figure 17. The parameters shown correspond to the reactive test problem that we previously solved with Baer's MOL code.

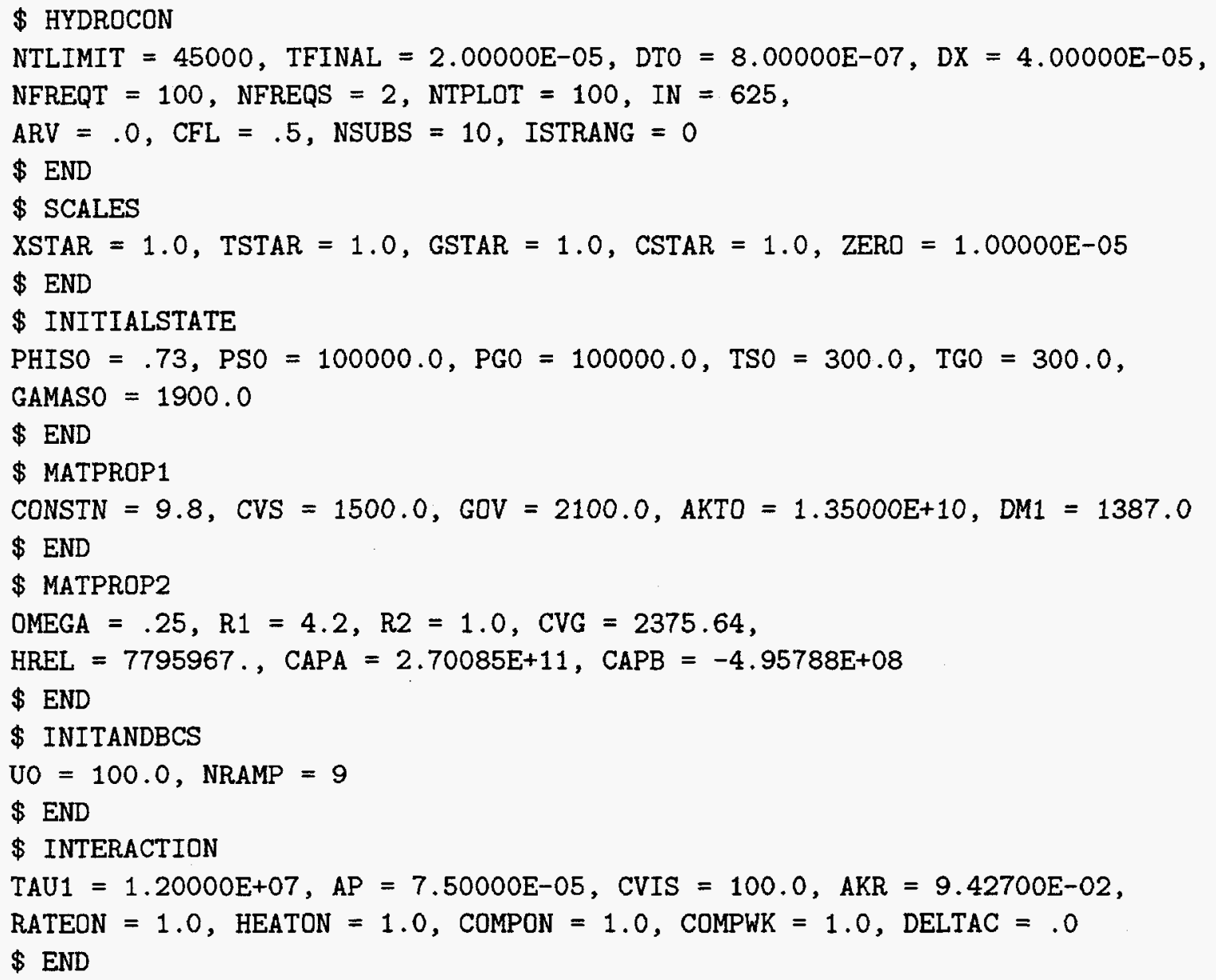

Fig. 17: The namelist output of the parameter values used for the BN-MOL and "SeptemberBKS" comparison run. This output can be found in the file "septout."

The results for this problem that were obtained using Baer's MOL code were displayed in Section 2 (see Figures 10-13). Presumably, the only difference between the BN and BKS 

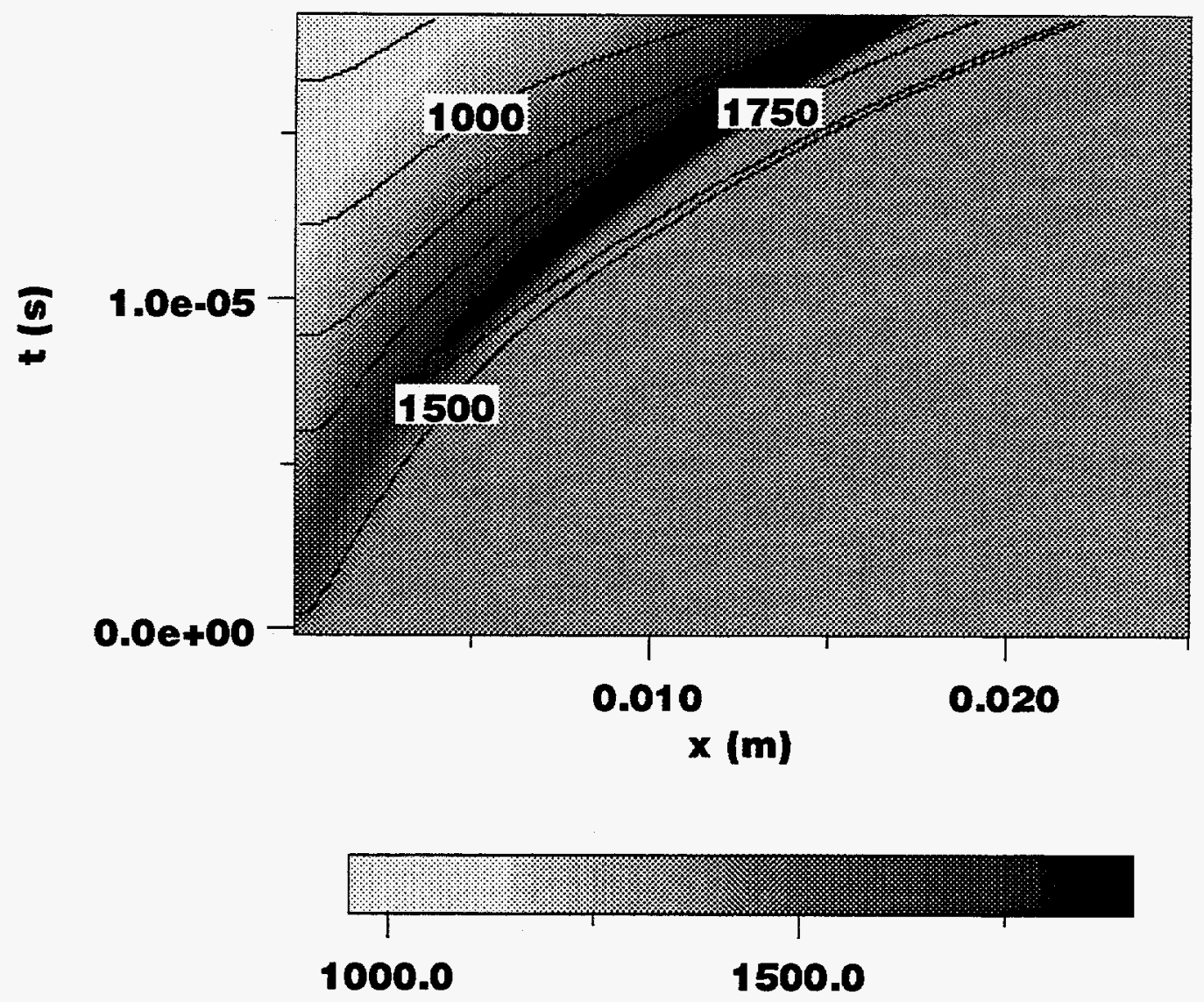

Fig. 18: A $t, x$-diagram showing $\left(\phi_{s} \rho_{s}\right)$ as computed with the BN-MOL code (the contour plot) and "September-BKS" (the gray-scale density plot). The reactive wave develops more quickly with the BN-MOL code.

solution is that BKS considers the limit of formally infinite drag. The parameters for the "September-BKS" numerics were 625 cells, 10 subcycles on the source terms, no artificial viscosity and a CFL value of 0.5. Doubling the number of nodes for Baer's code did not substantially change the results. Similarly, "September-BKS" was run with 1250 and 2500 cells with very little if any noticeable change.

A comparison of the results obtained with Baer's MOL code and "September-BKS" are shown in Figures 18 and 19. The most striking observation is that the two solutions do not compare very well at all. The BN-MOL code yields a considerably "hotter" solution; that is, the reactive wave develops more quickly and leads the "September-BKS" solution. A considerable amount of "code detective" work was done to verify both codes on problems with known solutions: (1) pure gas shock, (2) pure solid shock, and (3) a pure porous solid compaction wave. Both codes passed these tests. The differences seem to be related to the strong interaction that occurs between the phases in the reactive test problem.

There is reason to be suspicious of the accuracy of both solutions. Recall that the BKS, 


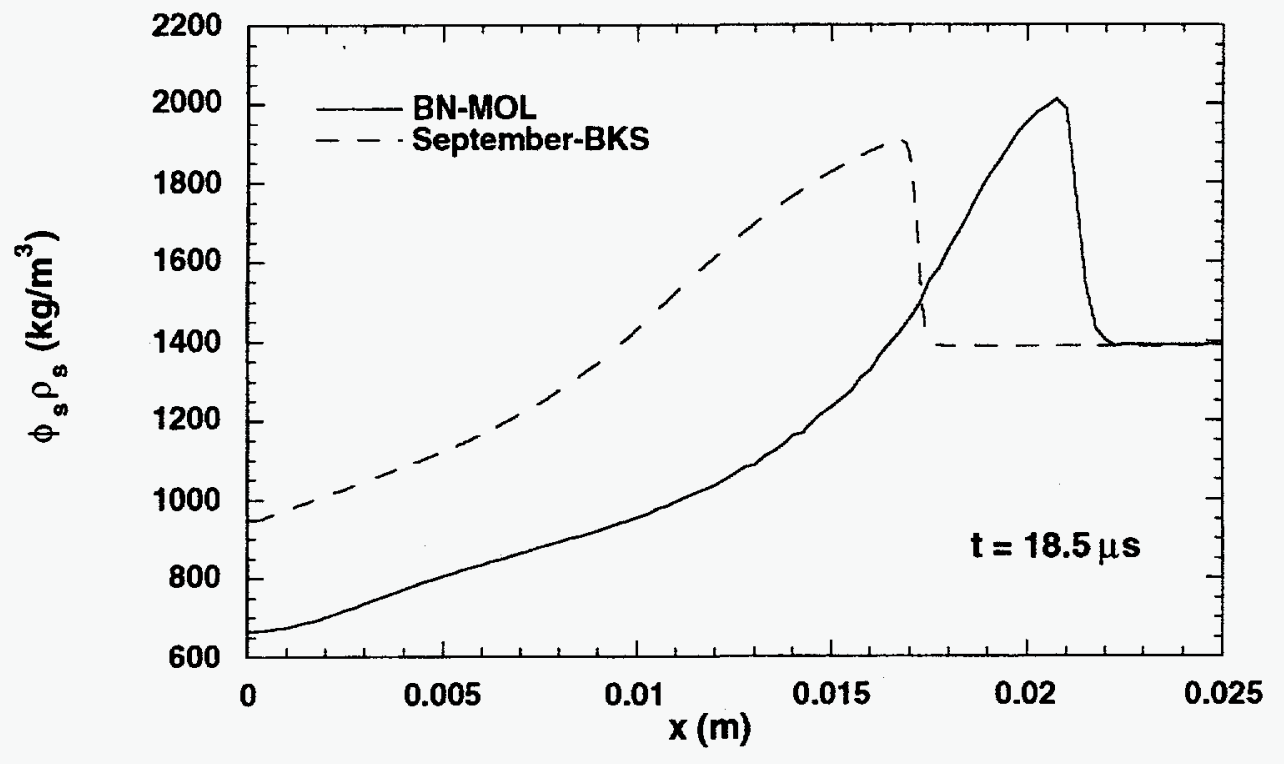

(a)

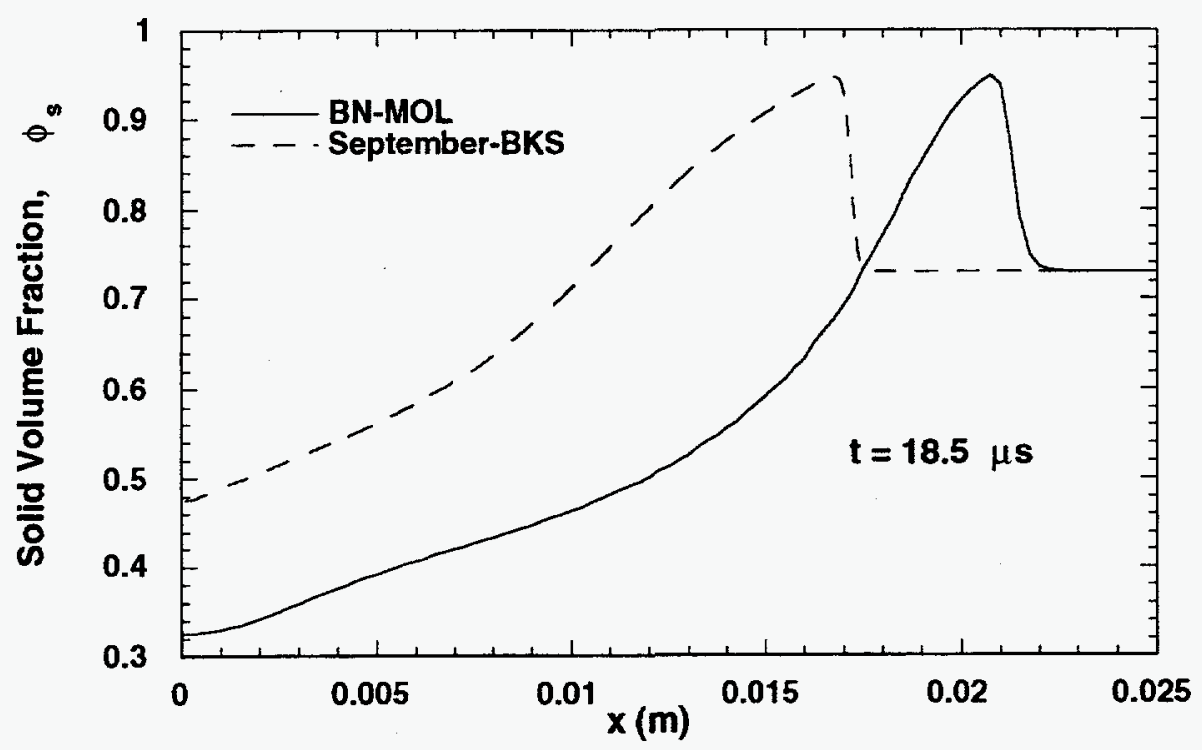

(b)

Fig. 19: A snapshot at $t=18.5 \mu s$ of $\left(\phi_{s} \rho_{s}\right)$, Part (a) and $\left(\phi_{s}\right)$, Part (b). The solution obtained with "September-BKS" is shown to lag. 
large-drag limit was formally derived by assuming that all gradients are $O(1)$. Clearly, this does not hold at the shock. Also the BKS model equations are not in conservation form. Perhaps the term displayed in Eq. (96) is not properly regularized by the numerical algorithm. The large value assumed by the drag given by Eq. (12) as $\phi_{s} \rightarrow 1$, represents a very narrow layer that is not resolved by Baer's MOL code. In addition, the interaction between $\delta$ and the large artificial viscosity used by Baer may produce problems. In any event, the solutions obtained with these two codes are radically different. This says that "September-BKS" does not connect to the old database. This casts a cloud of doubt over both solutions.

In order to help resolve this disagreement, a new fully two-velocity code, "September$2 \mathrm{~V}$ " was written and this test problem was run. To ensure that the effects of the large drag near shocks was adequately resolved, a constant value $\delta=10^{9} \mathrm{~kg} / \mathrm{m}^{3} / s$ was used. In this region $\left(V_{s}-V_{g}\right)=O(1)$, and the effects of drag are large. For more details on this region near the shock where $\left(V_{s}-V_{g}\right)=O(1)$ see Appendix B [7]. This region is called the dragrelaxation zone. The results of the comparison are shown in Fig. 20. The drag zone near the shock is clearly seen in these simulations. The gas phase velocity shown in (a) of Figure 20, clearly overshoots the solid velocity near the shock. After this near shock zone is traversed, the velocities are essentially identical for the remainder of the profile. The solid and gas phase pressure are shown in (b) of Figure 20. It is observed that the solid supports a steeply rising wave profile, while the gas pressure rises more slowly. These features are consistent with what is expected in a compactable granular material. Generally, the pressures are nearly equal. Figure 21 shows that the mixture velocity from "September-BKS" and solid velocity from "September-2V" compare very well. This is also true of the gas pressure and gas density (see Figures 21 (b) and 22). As noted for the BN-model solutions presented earlier, the phase densities and temperatures are very different for the two phases in these calculations.

No obvious numerical problems are expected for "September-2V" since the BN equations are in conservation form and the large drag effects are resolved in the calculation. The good comparison between "September-BKS" and "September-2V" suggest that, at least for this problem, the nonconservative term displayed in Eq. (96) is properly regularized by the numerics. This verifies BKS for this class of problems. Changing the drag term in "September-2V" to the expression given by Eq. (12) does not lead to any substantial differences.

Why the big discrepancy between Baer's MOL code and "September-2V"? When the issue of nonconservation form doesn't seem to lead to difficulty for this problem, one's focus shifts to the large artificial viscosity used in the MOL code (arv $=0.25$ ). When arv is reduced to arv $=0.125$, the code becomes stiff and crashes at about a problem time of $10 \mu \mathrm{s}$. Figures 12 (b) and 20 (b) clearly show that the shock pressure rise is considerably crisper in the "September-2V" solution. The discrepancies between BKS and BN need to be resolved; otherwise we are not doing good science. If Baer's solutions are somehow corrupted, that needs to be exposed. If coding errors exist in "September" they similarly need to be discovered and fixed. After many days of scrutinizing the code, we did not find a problem with "September."

As a part of the "September" verification exercise, we found, with the assistance of Ash Kapila, an analytical solution to the large-drag, shock relaxation zone problem (see 


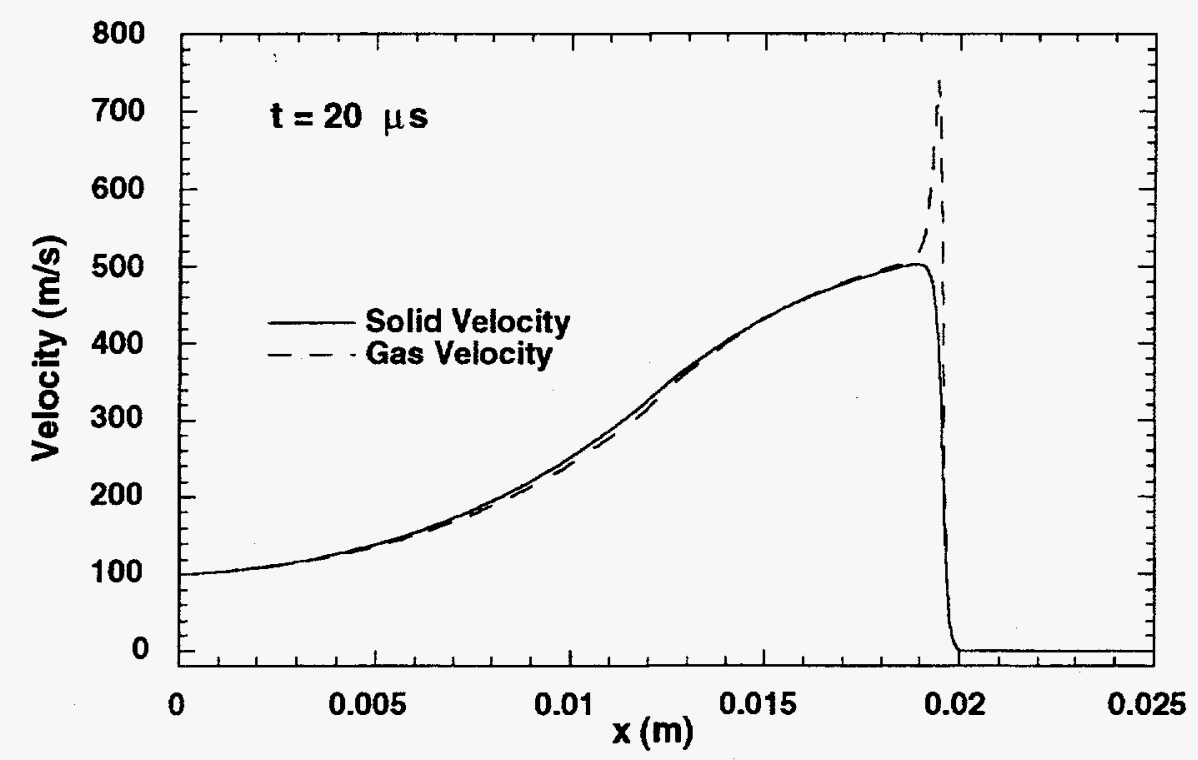

(a)

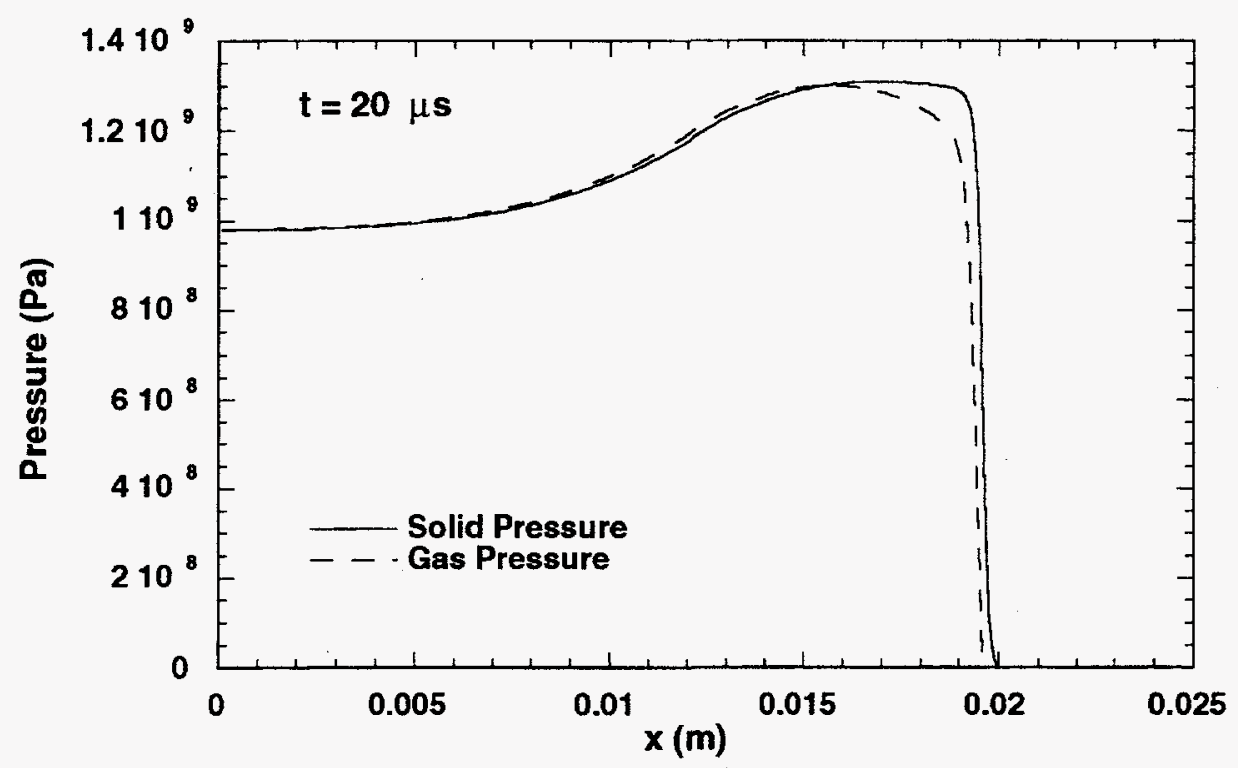

(b)

Fig. 20: A snapshot at $t=20 \mu \mathrm{s}$ of the "September-2V" solution of our reactive test comparison problem. The spike in the gas particle velocity is the result of the drag relaxation zone at the shock. $\delta=10^{9} \mathrm{~kg} / \mathrm{m}^{3} / \mathrm{s}$. 


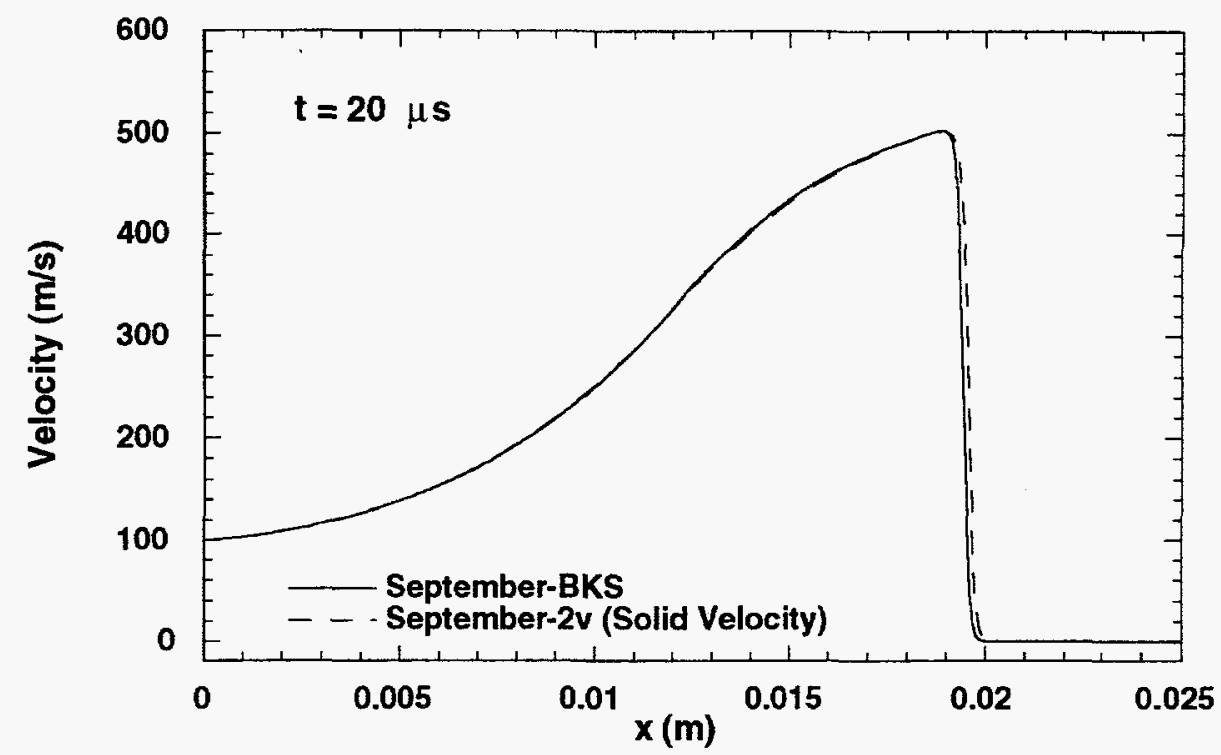

(a)

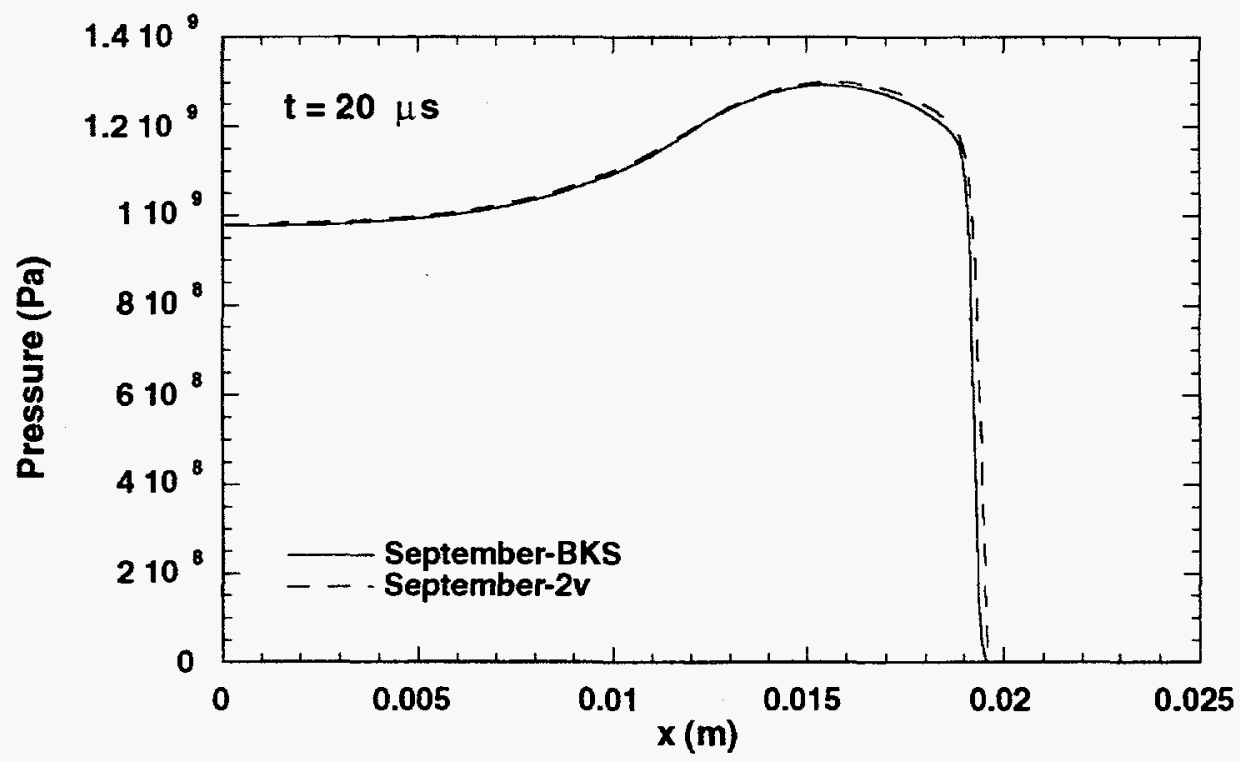

(b)

Fig. 21: A snapshot at $t=20 \mu s$ of the "September-2V" and the "September-BKS" solution to the reactive-wave test problem. The two solutions are in excellent agreement. 


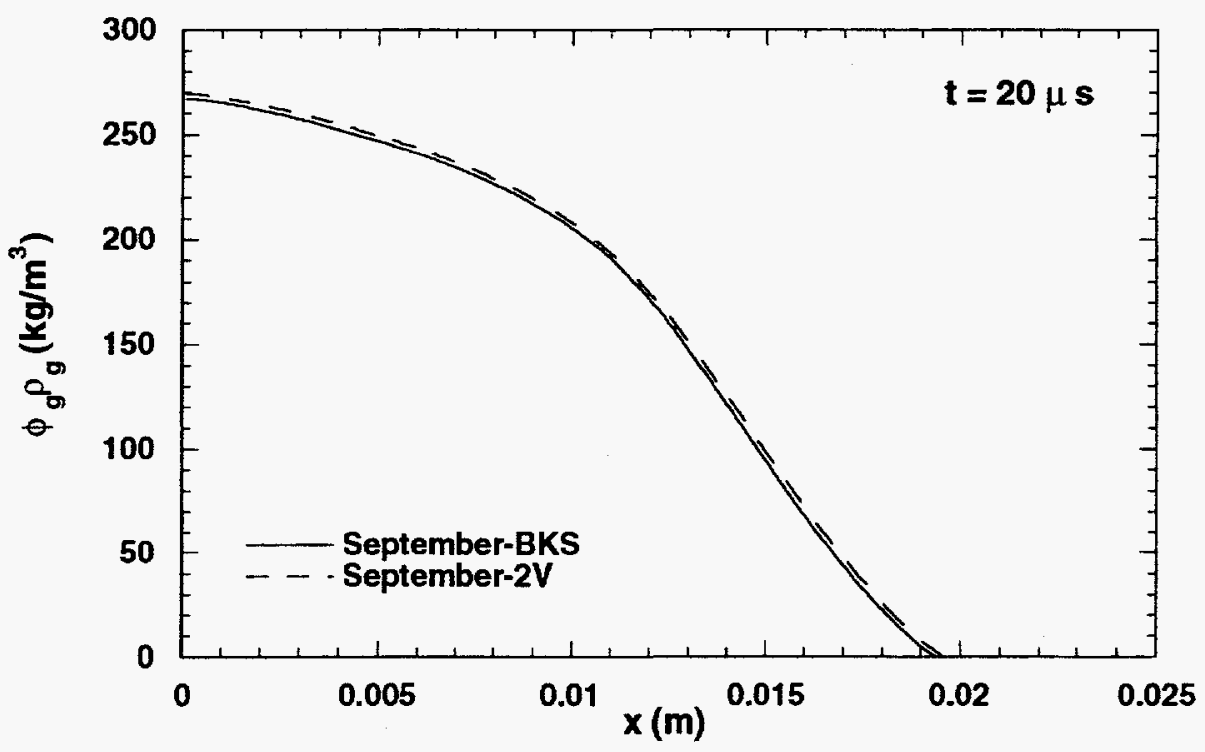

Fig. 22: A snapshot at $t=20 \mu s$ of $\left(\phi_{g} \rho_{g}\right)$ for the reactive-wave test problem obtained using "September-2V" and "September-BKS." The gas phase density is very low at the shock (near ambient). The variable $\left(\phi_{g} \rho_{g}\right)$ increases with the increasing progress of the reaction. 
Appendix B) [7]. The exact solution was compared with the solutions obtained numerically with "September-2V," "September-BKS" and the BN-MOL code. From the exact solution, we found that at shocks, $\left(V_{s}-V_{g}\right)=O(1)$ irrespective of the size of $\delta$. The size of $\delta$ controls only the thickness of the relaxation zone. Thus, at least formally, the BKS assumption that $\left(V_{s}-V_{g}\right)=O(1 / \delta)$ is incorrect. The problem considered in Appendix B retains only the drag interaction between the phases. When this exact solution was compared with "September-2V," excellent agreement was found. The "September-BKS" solution for the solid variables agreed with the exact solution reasonably well. However, errors were found in the computed "September-BKS" gas phase variables; particularly when the initial gas density was high. The BN-MOL code solutions showed the poorest overall agreement, and generally were fairly noisy.

\section{$5 \quad$ Reflections}

Where do we go from here? Early attempts to construct a reduced 7-equation BKS model look promising. By retaining a portion of the velocity-difference equation, Eq. (79) and a limited number of the velocity difference terms in Eqs. (72)-(77a, b), the proper entropy for the solid can be captured. The velocity difference is viewed as playing a role similar to that of an internal-state variable. It acts only in a very narrow region near the shock. The guiding principles used to construct this model are (1) the entropy of the solid is not allowed to change because of the added terms, and (2) the essential one-velocity character of the equations is not disrupted. This modified BKS model is

$$
\begin{aligned}
&\left(\phi_{s} \rho_{s}\right)_{t}+\left[\phi_{s} \rho_{s} V+\left(V_{s}-V_{g}\right)\left(\phi_{s} \rho_{s}\right)\left(\phi_{g} \rho_{g}\right) /\langle\rho\rangle\right]_{x}=C_{s}^{\dagger}, \\
&\left(\phi_{g} \rho_{g}\right)_{t}+\left[\phi_{g} \rho_{g} V-\left(V_{s}-V_{g}\right)\left(\phi_{s} \rho_{s}\right)\left(\phi_{g} \rho_{g}\right) /\langle\rho\rangle\right]_{x}=-C_{s}^{\dagger}, \\
&(\langle\rho\rangle V)_{t}+\left(\langle P\rangle+\langle\rho\rangle V^{2}\right)_{x}=0, \\
& {\left[\phi_{s} \rho_{s}\left(e_{s}+V^{2} / 2\right)\right]_{t}+\left[\phi_{s} \rho_{s} V\left(e_{s}+V^{2} / 2\right)+\phi_{s} P_{s} V\right]_{x}=} \\
&+V\left[\left(\phi_{g} \rho_{g}\right)\left(\phi_{s} P_{s}\right)_{x}-\left(\phi_{s} \rho_{s}\right)\left(\phi_{g} P_{g}\right)_{x}\right] /\langle\rho\rangle+\left(e_{s}+V^{2} / 2\right) C_{s}^{\dagger} \\
&-\left\{\left(P_{s}-\beta_{s}\left(\phi_{s}\right)\right) \mathcal{F}+\mathcal{H}\left(T_{s}-T_{g}\right)\right\} \quad \text { or }-\left\{P_{g} \mathcal{F}+\mathcal{H}\left(T_{s}-T_{g}\right)\right\} \\
&-\left(e_{s}+P_{s} / \rho_{s}+V^{2} / 2\right)\left[\left(V_{s}-V_{g}\right)\left(\phi_{s} \rho_{s}\right)\left(\phi_{g} \rho_{g}\right) /\langle\rho\rangle\right]_{x},
\end{aligned}
$$

and

$$
\begin{aligned}
{\left[\phi_{g} \rho_{g}\left(e_{g}+V^{2} / 2\right)\right]_{t} } & +\left[\phi_{g} \rho_{g} V\left(e_{g}+V^{2} / 2\right)+\phi_{g} P_{g} V\right]_{x}= \\
& -V\left[\left(\phi_{g} \rho_{g}\right)\left(\phi_{s} P_{s}\right)_{x}-\left(\phi_{s} \rho_{s}\right)\left(\phi_{g} P_{g}\right)_{x}\right] /\langle\rho\rangle-\left(e_{s}+V^{2} / 2\right) C_{s}^{\dagger} \\
& +\left\{\left(P_{s}-\beta_{s}\left(\phi_{s}\right)\right) \mathcal{F}+\mathcal{H}\left(T_{s}-T_{g}\right)\right\} \text { or }+\left\{P_{g} \mathcal{F}+\mathcal{H}\left(T_{s}-T_{g}\right)\right\} \\
& +\left(e_{s}+P_{s} / \rho_{s}+V^{2} / 2\right)\left[\left(V_{s}-V_{g}\right)\left(\phi_{s} \rho_{s}\right)\left(\phi_{g} \rho_{g}\right) /\langle\rho\rangle\right]_{x},
\end{aligned}
$$

and

$$
\begin{aligned}
\left(V_{s}-V_{g}\right)_{t} & +\left[V\left(V_{s}-V_{g}\right)\right]_{x}= \\
& +\langle\rho\rangle\left[P_{g}\left(\phi_{s}\right)_{x}-\delta\left(V_{s}-V_{g}\right)\right] /\left(\phi_{s} \rho_{s}\right) /\left(\phi_{g} \rho_{g}\right) \\
& +\left(\phi_{g} P_{g}\right)_{x} /\left(\phi_{g} \rho_{g}\right)-\left(\phi_{s} P_{s}\right)_{x} /\left(\phi_{s} \rho_{s}\right) .
\end{aligned}
$$


This 7-equation model adds an equation for the velocity difference. However, advection continues to occur at the baracentric velocity $V$. The coupling to the remaining equations is principally through the two mass conservation equations; these equations maintain their full two-velocity form. Equation (98a, b) says that except for compaction, heat transfer and shocks, the solid is isentropic. The addition of the $\left(V_{s}-V_{g}\right)$ terms in Eqs. (105) and (106) will violate the constant solid entropy required by Eq. (98a, b) unless terms are added in Eqs. (108a, b) and (109a, b); the added terms ensure that the solid entropy remains constant after the shock is crossed. Because the added terms are all proportional to $\left(V_{s}-V_{g}\right)$, these modifications only influence the relaxation zone where $\left(V_{s}-V_{g}\right) \neq 0$. These added terms regularize the shock by resolving the relaxation structure.

These equations are coded in "September-1V." The results for our reactive test problem, using $\delta=10^{8} \mathrm{~kg} / \mathrm{m}^{3} / \mathrm{s}$, are shown in Figure 23. The added terms and equation spread out the shock, thereby regularizing the system of equations. The wave speed is not affected and the profiles look similar to those obtained with BKS.

The extended-BKS model represents but one of many possibilities for regularizing the BKS model at shocks. The above approach continues to rely on the $\mathrm{BN}$ equations for the physical description of the momentum exchange at shocks; in the form of the velocitydifference equation, Eq. (110). Because slow flow, Stokes-like drag $\delta\left(V_{s}-V_{g}\right)$ is not a particularly good representation for high-speed flows with shocks, other forms need to be tried. For example, completely different closures, such as

$$
\delta\left(V_{s}-V_{g}\right) \Longrightarrow \alpha \frac{\partial^{2} V}{\partial x^{2}}
$$

need to be considered. Kapila and Menikoff are looking at this problem.

For the past two years, we've worked on selecting the basic hydrodynamic structure of the model (i.e., one-phase or two-phase, one-velocity or two-velocity). It's now time to make a choice and proceed with modeling the "chemistry." The success of our modeling will largely be determined by the quality of our "chemistry" modeling.

Our preference is a two-phase, one-velocity model with two temperatures and two pressures. Most everything that we know about reactive waves in heterogeneous, energetic materials argues for the importance of energy localization and microscale "hotspots." We would find it very difficult to think of these problems without two or possibly three temperatures. This goes back to Don Drew's rule; "always think about your system at one higher level of resolution than the level of your modeling" [32]. Our goal in the explosives safety problem is to successfully calculate accident scenarios that are very different from the limited number of calibration experiments that can be performed. 


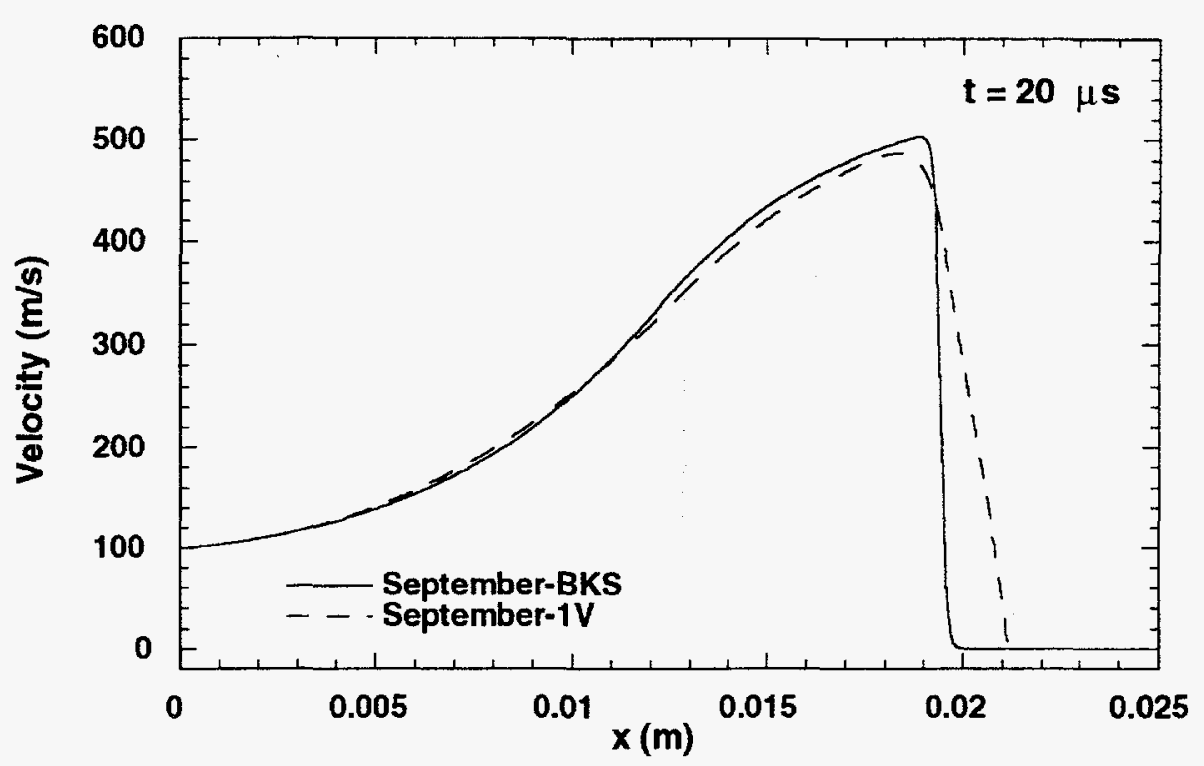

(a)

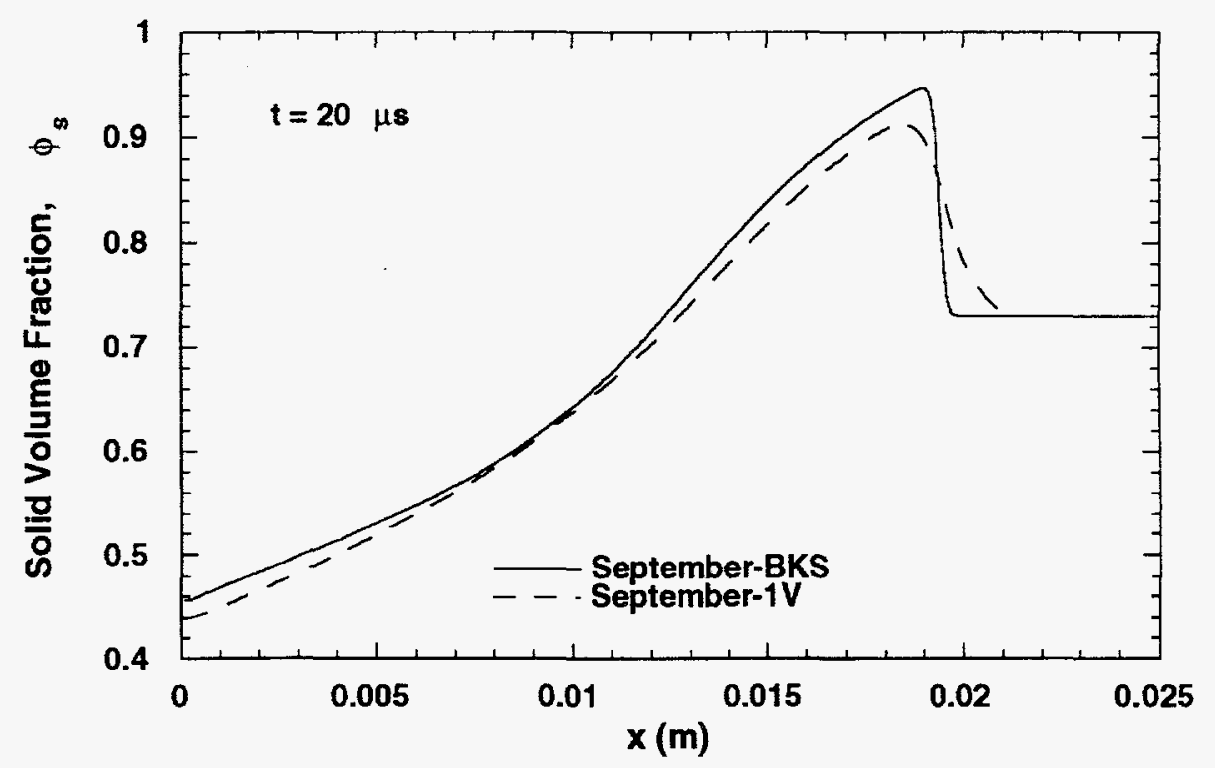

(b)

Fig. 23: A snapshot at $t=20 \mu s$ of the particle velocity $V$ in Part (a) and the compaction $\phi_{s}$ in Part (b). The rapidly rising profiles are obtained from BKS and the ramped rises are obtained with our ad hoc 7 -equation model. 


\section{Acknowledgments}

This report gives an overview of the work performed by the theory and modeling group of the Los Alamos DDT program, from 1992-94. We would especially like to thank the following list of participants for their contributions: Blaine Asay, Shirish Chitanvis, Bill Davis, Dave Funk, Rick Gustavsen, Larry Hill, Phil Howe, Ash Kapila (RPI), Ed Kober, Gary Laabs, Roy Lucht, John McAfee, Ralph Menikoff, Kuldeep Prasad (Yale), Steve Sheffield, Scott Stewart (U of I), and Shaojie Xu (U of I). We thank Mel Baer (SNLA) for providing us with a copy of his BN-MOL code, for help running and modifying BN-MOL, and for helpful discussions on the theory of two-phase flow.

\section{References}

[1] B. Asay and L. Hantel, "Major Thrust Areas for Examination of Deflagration-toDetonation Transition in Granular and Damaged Explosives," Los Alamos National Laboratory memo M-8-91-61 (1991).

[2] C. L. Mader, Numerical Modeling of Detonations, Univ. of California Press, Berkeley, CA, (1979).

[3] M. Baer and J. Nunziato, "A Two-Phase Mixture Theory for the Deflagration to Detonation Transition (DDT) in Reactive Granular Materials," Int J. Multiphase Flow, Vol. 12, No. 6, pp. 861-889, (1986).

[4] S. A. Sheffield, R. L. Gustavsen and R. R. Alcon, "Shock Initiation Studies of Low Density HMX Using Electromagnetic Particle Velocity and PVDF Stress Gauges," Tenth (International) Symposium on Detonation, Boston, MA, July 12-16, 1993, (1993).

[5] M. Baer, "A Model for Interface Temperature Induced by Convective Heat Transfer in Porous Materials," SAND 88-1073, Sandia National Laboratories, (1989).

[6] J. M. Powers, D. S. Stewart and H. K. Krier, "Theory of Two-Phase Detonation-Part 1: Structure," Combust. Flame, Vol. 80, pp. 280-303, (1990).

[7] S. F. Son, "Comments on the Large-Drag Reduction of the Baer-Nunziato (BN) Model," Los Alamos National Laboratory memo DX-16:94-99, (1994).

[8] D. Kunii and O. Levenspiel, Fluidization Engineering, Robert E. Krieger Press, Huntington, NY, (1977).

[9] W. Herrmann, "Constitutive Equations for the Dynamic Compaction of Ductile Porous Materials," J. Appl. Phys., Vol. 40, No. 6, pp. 2490-99, (1969).

[10] R. H. Wentorf, Jr., Advances in High-Pressure Research, Vol. 4, Academic Press, New York, (1974).

[11] P. J. Coyne, W. L. Elban and M. A. Chiarto, "The Strain Rate Behavior of Coarse HMX Porous Bed Compaction," Eighth Symposium (International) on Detonation, Albuquerque, NM, July 15-19, 1985, pp. 645-57, (1985). 
[12] W. L. Elban and M. A. Chiarito, "Quasi-Static Compaction Study of Coarse HMX Explosive," Powder Technology, Vol. 46, pp. 181-93, (1986).

[13] M. U. Anderson, R. A. Graham and G. T. Holman, "Time-Resolved Shock Compression of Porous Rutile: Wave Dispersion in Porous Solids," APS Topical Group on Shock Compression, Colorado Springs, CO, June 28 - July 2, 1993, (1993).

[14] D. J. Benson and W. J. Nellis, "Numerical simulation of the shock compaction of Copper powder," in High-Pressure Science and Technology-1993, the proceedings of the joint International Association for Research and Advancement of High Pressure Science and Technology and APS Topical Group on Shock Compression of Condensed Matter Conference held at Colorado Springs, CO, June 28-July 2, 1993, AIP Conference Proceedings 309, Part 2, AIP Press, New York, pp. 1243-1246 (1993).

[15] J. B. Bdzil, "Calculation of the LANL/SNLA Gun Experiments Performed on Porous $\phi_{s}{ }^{\circ}=0.73$ HMX," Los Alamos National Laboratory Quarterly Report M-7-QR-93-1, (1993).

[16] P. Embid and M. Baer, "Mathematical Analysis of a Two-Phase Model for Reactive Granular Material," Sandia National Laboratories report SAND 88-3302, (1989).

[17] P. Embid, J. Hunter and A. Majda, "Simplified Asymptotic Equations for the Transition to Detonation in Reactive Granular Materials," SIAM J. Appl. Math., Vol. 52 (5), pp. 1199-1237, (1992).

[18] W. Fickett and W. C. Davis, Detonation, Univ. of California Press, Berkeley, CA, (1979).

[19] D. S. Stewart, S. Xu, A. K. Kapila, J. B. Bdzil and S. F. Son, "The September-BKS DDT Code," private communication, Los Alamos National Laboratory (1993).

[20] A. K. Kapila and J. B. Bdzil, "On Steady Compaction Waves in the Baer-Nunziato model," private communication, Los Alamos National Laboratory (1992).

[21] J. E. Shepherd and D. R. Begeal, "Transient Compressible Flow in Porous Materials," Sandia National Laboratoies report SAND 83-1788, (1988).

[22] B. Asay, D. Funk and G. Laabs, "Measurement of High-Pressure Gas Permeation of $\phi_{s}{ }^{\circ}=0.73$ Granular SiC," Los Alamos National Laboratories, private communication (1992).

[23] M. Cowperthwaite, "The Blake/Tiger Thermochemistry Code," Ver. 205.11, SRI International, (1988).

[24] M. Baer, BN-MOL code, Sandia National Laboratories, private communication, (1992).

[25] D. S. Stewart, B. Asay and K. Prasad, "Simplified Modeling of Transition to Detonation in Porous Energetic Materials," Phys. Fluids, Vol. 6, No. 7, pp. 2515-2533, (1994).

[26] A. K. Kapila, "Comments on the Development of the BKS Model," private communication, Rensselaer Polytechnic Institute (1993-1994). 
[27] J. M. McAfee, B. Asay, W. Campbell and J. B. Ramsay, "Deflagration to Detonation in Granular HMX," Ninth Symposium (International) on Detonation, Portland, OR, Aug. 28 - Sept. 1, 1989, pp. 265-79, (1989).

[28] S. F. Son, "Preliminary Results: Plug Formation," Los Alamos National Laboratory memo DX-16:94-17, (1994).

[29] G. I. Kerley, "CTH Equation of State Package: Porosity and Reactive Burn Models," Sandia National Laboratories report SAND 92-0553, (1992).

[30] K. S. Holian, D. A. Mandell, T. F. Adams, F. L. Addessio, J. R. Baumgardner and S. J. Mosso, "MESA: a 3-d Computer Code for Armor/Anti-armor Applications," Supercomputing World Conference, San Francisco, CA, Oct. 17-20, 1989, (1989).

[31] A. K. Kapila, "Asymptotic Reduction of Two-Phase DDT Models: A Writeup of 4/93 DDT Meeting Presentation," private communication, Rensselaer Polytechnic Institute (1993).

[32] D. Drew, "Summary of Los Alamos DDT Program Review," Los Alamos National Laboratory memo M-8-92-131, (1992). 


\section{Appendix A}

\section{CALCULATION OF GUN EXPERIMENTS}

Los Alamos National Laboratory Quarterly Report M-7-QR-93-1 


\section{Calculations of the LANL/SNLA Gun Experiments Performed on Porous $\phi_{S O}=0.73$ HMX \\ (John Bdzil)}

LANL/SNLA did two experiments of the shock initiation variety on $\phi_{s o}=0.73$ porous HMX. The nominal sample thickness was $\sim 4 \mathrm{~mm}$. The experiments were carried out in a sandwich geometry.

\begin{tabular}{|c|l|c|c|c|}
\hline $\begin{array}{c}\text { Kel-F } \\
\text { or } \\
\text { PMMA } \\
\text { Projectile }\end{array}$ & $\rightarrow$ & $\begin{array}{c}\text { Kel-F } \\
\text { or } \\
\text { PMMA }\end{array}$ & $\begin{array}{c}\text { porous } \\
\text { HMX }\end{array}$ & $\begin{array}{c}\text { Kel-F } \\
\text { or } \\
\text { PMMA }\end{array}$ \\
\hline
\end{tabular}

The projectile speeds were

Kel-F: $\quad 390 \mathrm{~m} / \mathrm{s}$

PMMA: $\quad 700 \mathrm{~m} / \mathrm{s}$.

Both particle velocity and pressure were measured at the two interfaces, $\mathbf{0}$ and 2 (the pressure measurements @ interface 2 were of very short duration).

The object of this study (as originally conceived) was to determine if Baer's work ${ }^{[1]}$ provided us with a good estimate for a detonation ignition rate law. The plan was to use the LANL model of DDT as a framework for thinking about the transition process and decompose the problem into a low pressure ignition regime [(1) the Shepherd test] and a piston supported detonation ignition regime [(2) the LANL/SNLA gun experiments]. The idea was that these two regimes communicate with one another through an impermeable, semi-inert plug that acts like a piston. What I will do here is report on my calculations of two detonation experiments using Baer's DDT model.

To do these calculations I had to further modify Baer's code to do the most complete example of multimaterials; nonporous inerts interacting via an interface with porous explosive.

\begin{tabular}{c|c}
\hline $\begin{array}{c}\text { nonporous } \\
\text { inert } \\
\text {-one phase- }\end{array}$ & $\begin{array}{c}\text { porous } \\
\text { HMX } \\
\text {-two phase- }\end{array}$ \\
\hline $\begin{array}{c}\text { solid-phase } \\
\text { only }\end{array}$ & $\begin{array}{c}\text { solid and } \\
\text { gas phase }\end{array}$
\end{tabular}


In regions with pure solid I solved only the solid phase equations, setting all interaction terms with the gas to zero. In solid phase regions the evolution of the gas phase variables was set to zero. The HMX was modeled in the standard fashion following Baer. ${ }^{[1]}$

The interface between these two regions was constrained to move at the common solid velocity, $V_{s}$ (same for inert and solid HMX). The velocity of the gas, $V_{g}$ : at the interface was constrained to be equal to that of the solid according to the two mass and momentum equations. To get balancing of mixture pressure (i.e., $P_{m i x}=\phi_{g} P_{z}+\phi_{s} P_{s}$ ) across the interface required special treatment of the nonconservative nozzling term in Baer's mechanics model. The integral of

$$
P_{g} \frac{\partial \phi}{\partial x}
$$

across the interface was constrained to be

$$
\left.P_{g}\right|_{H E}\left(\left.\phi_{S}\right|_{H E}-\left.\phi_{S}\right|_{\text {in }}\right)=\left.P_{g}\right|_{H E}\left(\left.\phi_{S}\right|_{H E}-1\right)
$$

Enforcing this condition leads to a balancing of the mixture pressure across the interface. This produced a sensible matching of the two phase and one phase models across the interface.

These calculations showed a number of interesting features. First the inert material response of the porous HMX produced interesting structure in both $V$ and $P$ (at the interface) that is related to the finite time required for the porosity to collapse. For example, the $V$ and $P$ profiles at the input interface of the HMX showed an initial response that was "higher" impedance that then relaxed to a "lower" impedance when the porosity collapsed. The (PMMA/inert HMX) interface match produced the following particle velocity, $V$ and pressure, $P$ response at interface 0 (See Figs. $1 \mathrm{a}$ and $1 \mathrm{~b}$ )
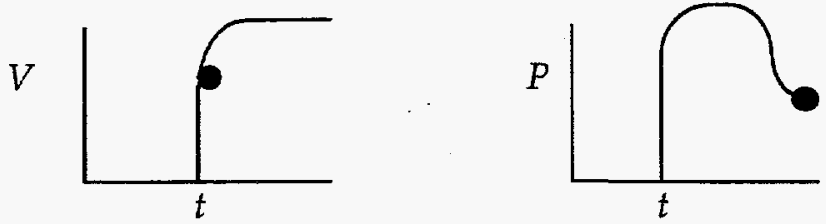

whereas when porosity collapse was inhibited the response was
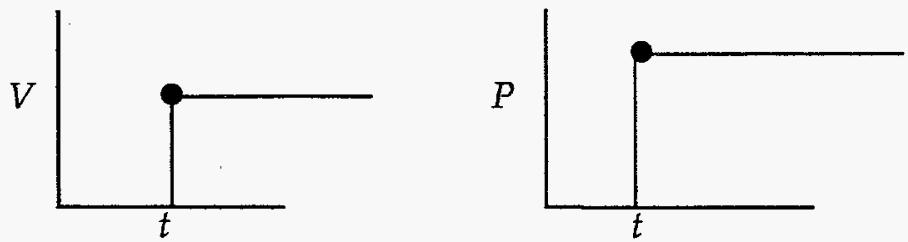
(rate-dependent compaction)
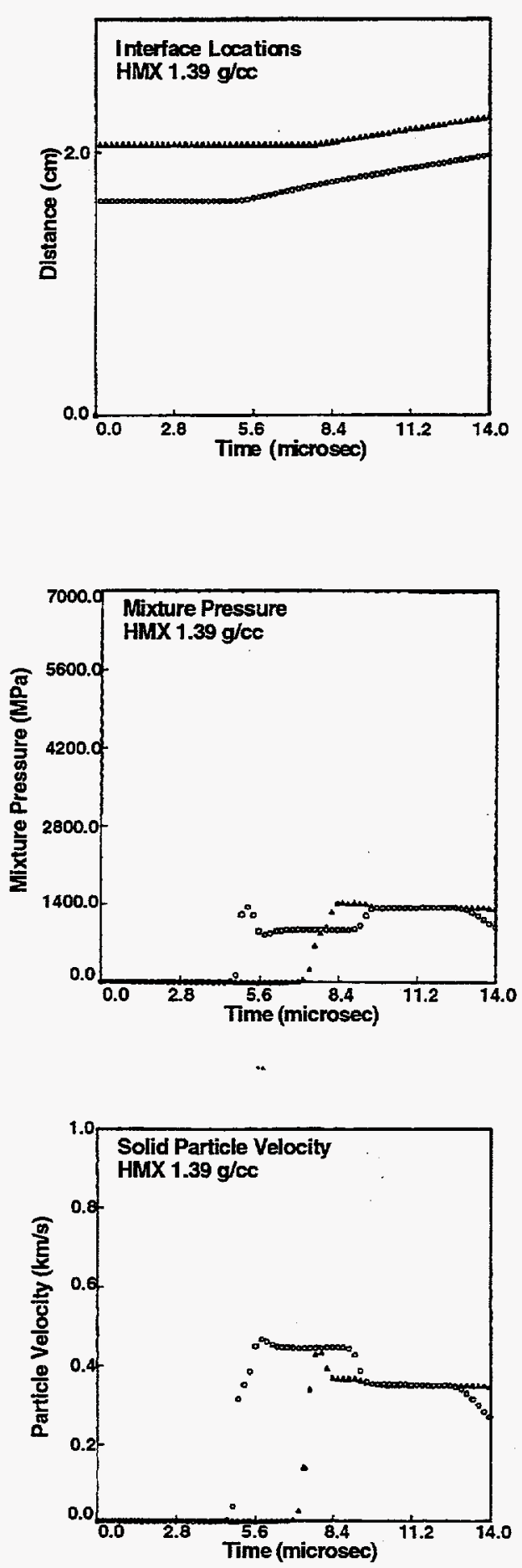

(no compaction)
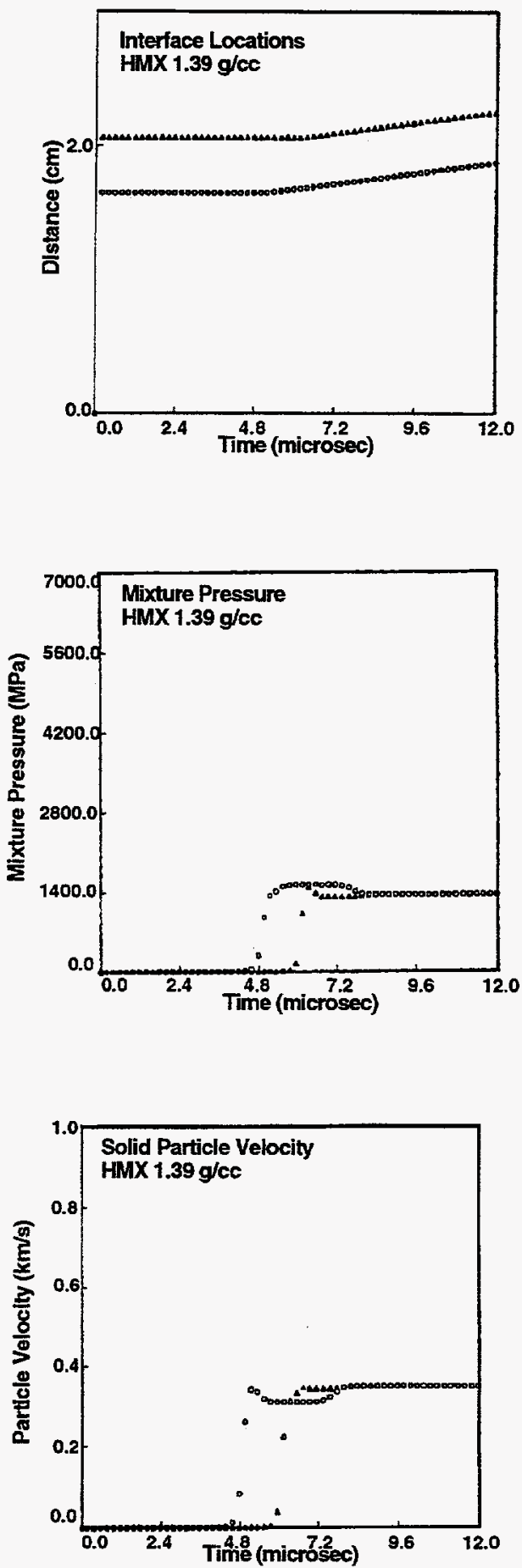

Figure 1a. Case I: PMMA/inert-HMX/PMMA - $700 \mathrm{~m} / \mathrm{s}$ projectile velocity 


\section{(rate-dependent compaction)}
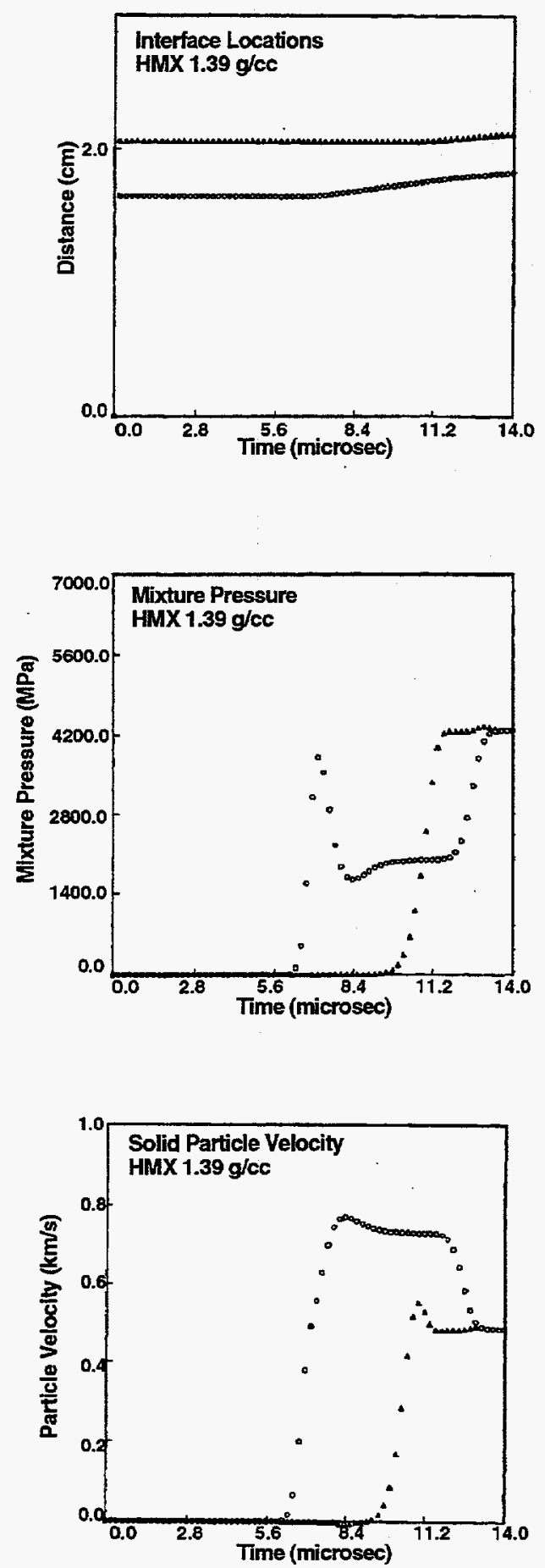

(no compaction)
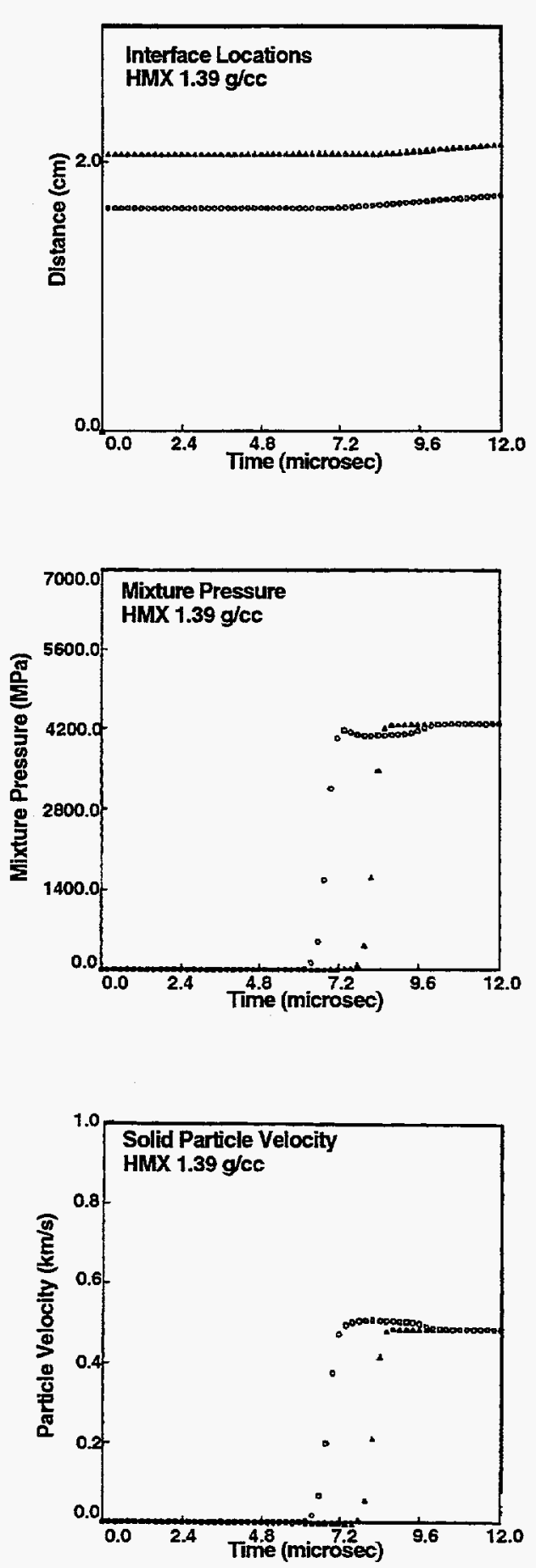

Figure 1b. Case II: Kel-F/inert--HMX/Kel-F - $390 \mathrm{~m} / \mathrm{s}$ projectile velocity 
These features are controlled by the crush-up dynamics of the porous HMX and have little to do with the viscoelastic response of the PMMA. Although the artificial viscosity produces considerable numerical smearing of "sharp" fronted waves, it is clear that the compaction viscosity $\left(\mu_{C}\right)$ appearing in the following equation is too large:

where

$$
\frac{D \phi_{s}}{D t}=\frac{\phi_{g} \phi_{s}}{\mu_{c}}\left(\operatorname{delp}-P_{g}+P_{i n t}\right)
$$

and

$$
\operatorname{delp}=\operatorname{amax}\left(0.0,\left(P_{S}-P_{m c}\right)\right)
$$

$$
P_{m c}=\text { intergranular stress, } P_{\text {int }}=\text { ambient pressure } \text {. }
$$

That is, Baer's value of $\mu_{c}=10^{3}$ is too large. Generally speaking, the overall (after the initial transient) matching of $V$ and $P$ at the input interface was in fair agreement with the experiment (when reactive effects are considered).

From these results it is clear that if we believe that the crush up dynamics is important, then some experiments need to be directed specifically at this effect. The experiments that have been done to this point do not speak clearly on this issue. At the very least, we as a group need to consider this issue carefully. I will now go on to consider the reactive material response of the HMX.

Baer has calibrated a three-step reaction model for porous HMX whose initial state of compaction is $\phi_{s o}=0.73$. This rate model was calibrated to reproduce Sandusky's $x, t-$ data and pressure data on $\phi_{s o}=0.73$ class D HMX. ${ }^{[2]}$ Sandusky studied the response of porous HMX confined in a DDT tube to low-velocity $(\sim 100 \mathrm{~m} / \mathrm{s})$ sustained impacts. Here I examine the ability of this rate model (the only one currently calibrated for low-velocity impacts) to reproduce the LANL/SNLA gun experiments. As the previous inert material calculations showed, the dynamics associated with this experimental geometry are themselves complicated. To help us think about the "chemistry," I will write down the important parts (i.e., functional parts) of Baer's "chemistry" model:

\section{mass conservation}

$$
\begin{aligned}
& \underline{\underline{\text { gas }}} \quad \begin{array}{c}
\frac{\partial}{\partial t}\left(\phi_{g} \gamma_{g}\right)+\frac{\partial}{\partial x}\left(\phi_{g} \gamma_{g} V_{g}\right)=-C_{s}^{+} \\
\underline{\underline{\text { solid }}}
\end{array} \frac{\partial}{\partial t}\left(\phi_{s} \gamma_{s}\right)+\left\{\frac{\partial}{\partial x}\left(\phi_{s} \gamma_{s} V_{s}\right)\right\} * \text { Unit }=C_{s}^{+} * \text { Unit }
\end{aligned}
$$

compaction(porosity)

$$
\frac{\partial}{\partial t}\left(\phi_{s}\right)+\left\{V_{s} \frac{\partial}{\partial x}\left(\phi_{s}\right)\right\} * \text { Unit }=\left\{\left(\phi_{S} \phi_{g} / \mu_{c}\right)\left(\text { delp }-P_{g}+P_{\text {int }}\right)+\left(C_{s}^{+} / \gamma_{s}\right)\right\}^{* \text { Unit }}
$$

"hot-spot" reaction progress variable $\equiv P_{y}$ 
where

$$
\frac{\partial}{\partial t}\left(P_{y}\right)+\frac{\partial}{\partial x}\left(P_{y} V_{g}\right)=\operatorname{dreact} *\left(1-P_{y} / \phi_{g} \gamma_{g}\right) * \phi_{g} \gamma_{g}-P_{y} * C_{s}^{+} / \phi_{g} \gamma_{g}
$$

$$
\begin{aligned}
& \text { dreact }=\left(0.2 \times 10^{-13}\right) *\left(P_{\text {mix }}-P_{\text {int }}\right)^{2} \\
& P_{\text {mix }}=\phi_{g} P_{g}+\phi_{S} P_{S}
\end{aligned}
$$

with $P_{y}=0.0 @ t=0$, and

"grain surface" temperature function $\equiv$ fh

$$
\frac{\partial}{\partial t}(f h)+\frac{\partial}{\partial x}\left(f h V_{s}\right)=3 * h *\left(T_{g}-T_{S_{\text {surface }}}\right) * \phi_{s} / C_{v s} a+f h * C_{s}^{+} / \phi_{s} \gamma_{s}
$$

where

$T_{s}$ surface $\left(f h, T_{g}, T_{S}\right)=$ an algebraic function describing the surface temperature

$\mathrm{h}=$ convective heat transfer coefficient

$\mathrm{a}=$ particle (solid) radius

with $f h=0.0 @ t=0$. CGS units are used throughout. A JWL form is used for the reaction products (gas), with

$$
\begin{aligned}
& T_{g}=T_{g 0}+E n g y / C_{v g} \\
& \text { Engy }=e_{g}+\text { hrel }-\left(\left(A / R_{1}\right) \exp \left(-R_{1} v\right)+\left(B / R_{2}\right) \exp \left(-R_{2} v\right)\right) /\left(\phi_{S o} \gamma_{S}^{0}\right)
\end{aligned}
$$

where

$$
\begin{aligned}
& v=\frac{\phi_{s a} \gamma_{S}^{o}}{\gamma_{g}} \\
& \text { hrel }=h p y r *(1-\text { delay })+h \operatorname{det}^{*} \text { delay } \\
& \uparrow \text { heat of } \uparrow \text { heat of } \\
& \text { pyrolsis detonation }
\end{aligned}
$$

The "hot spot" reaction variable $P_{y}$ triggers a discontinuous heating of the gas when $\left(P_{y} / \phi_{g} \gamma_{g}\right)$ exceeds 0.5 , with

$$
\begin{array}{ll}
\text { delay }=0.0, & \left(P_{y} / \phi_{g} \gamma_{g}\right)<0.5 \\
\text { delay }=1.0, \quad\left(P_{y} / \phi_{g} \gamma_{g}\right) \geq 0.5 .
\end{array}
$$

The algebraic function $T_{S}$ surface relates the surface temperature of solid $\left(T_{S}\right.$ surface $)$ to $f h$, $T_{g}, T_{S}$. The large scale bulk burning of the HE (governed by $C_{s}^{+}$) is turned on when $T_{S_{\text {surface }}}>T_{\text {ign }}=525^{\circ} \mathrm{K}\left(\right.$ for $\left.\phi_{\text {SO }}=0.73\right)$. Now,$C_{s}^{+}$is 


$$
\begin{aligned}
C_{S}^{+}=-\gamma_{S} * A_{\text {coef }} *\left(\left(P_{g} * 10^{-9}\right) * * b n\right) * 3 * \phi_{S} * e t i *\left(1-\frac{\phi_{S}-\phi_{S 0}}{1-\phi_{S 0}}\right)^{\frac{2}{3}} \frac{1}{a} ; \text { major reaction } \\
-A l_{\text {star }} * \frac{\phi_{S}-\phi_{S 0} * \gamma_{s} *\left(1-\phi_{S 0}\right) ; " \text { hot - spot" like reaction }}{1-\phi_{S 0}}
\end{aligned}
$$

where

$$
\begin{aligned}
& \text { eti }=\text { CVMGT }\left(0.0,1.0 ; T_{\left.s_{\text {surface }} . l t . T_{\text {ign }}\right)}\right. \\
& A l_{\text {star }}=100 * *\left(\left(P_{\text {mix }}-P_{\text {int }}\right) * 10-9\right) * * 2 \\
& A_{\text {coef }}=11.39, b n=0.8 \quad P_{g}<6.9 \times 10^{8} \\
& A_{\text {coef }}=12.27, b n=1.0 \quad P_{g} \geq 6.9 \times 10^{8} .
\end{aligned}
$$

The variables $\left(P_{y}\right)$ and $(f h)$ serve as triggers for the major reaction component of $C_{s}^{+}$, which governs how the vast majority of solid is converted to gas. I will now describe how the triggering reactions that preceed the major energy releasing reaction operate for Baer's HMX model for the two cases considered in Figs. 1a and 1b. Case II (Kel-F inert with a 390 $\mathrm{m} / \mathrm{s}$ projectile velocity) is considered first.

\section{Case II. (Kel-F/HMX/Kel F - $390 \mathrm{~m} / \mathrm{s}$ projectile velocity)}

For this case, the Baer model and experiment look qualitatively similar. Vigorous reaction is initiated on wave reflection off the 2 nd $\mathrm{HMX} / \mathrm{Kel}-\mathrm{F}$ interface, where the pressure jumps to roughly $10 \mathrm{kbar}$. Both the calculated and experimental response of the 1st Kel-F/HMX interface show the finite rate compaction process playing a role. The initial spike in the pressure at the 1st $\mathrm{HMX} / \mathrm{Kel}-\mathrm{F}$ interface is a feature of both the calculation and the experiment. Based on these results, I suggest that the nonideal response of the porous $\mathrm{HMX}$ has more to do with the rounding of the 1 st $\mathrm{HMX} / \mathrm{Kel}-\mathrm{F}$ particle velocity history than does the viscoelastic response of the Kel-F. Clearly porous HMX is a very nonideal material. It is clear from these calculations that Baer's compaction viscosity is too large.

Instead of the constant particle velocity shown in the experiment, the calculated particle velocity at the 1st $\mathrm{HMX} / \mathrm{Kel}-\mathrm{F}$ interface decreases after the compaction response. This implies a weak energy release is occurring in the calculation. Interestingly enough, calculation and experiment are in better agreement for the pressure, with both showing evidence of weak reaction.

When the computational results are examined with an eye towards understanding the reaction scheme, I find the following sequence of events:

(1) The weak "hot-spot" like reaction component of $C_{s}^{+}$ 
$-A l_{s t a r} *\left(\frac{\phi_{s}-\phi_{s 0}}{I-\phi_{s 0}}\right) * \gamma_{s} *\left(1-\phi_{s o}\right)$

generates both additional gas and an increased entropy, hotter gas than that obtained in a purely isentropic process;

(2) this raises the gas to $\sim 2000 \mathrm{~K}$, which then forces the grain surface temperature, $T_{S}$ surface to exceed the critical value of $525 \mathrm{~K}$. This forces $C_{S}^{+}$to become large, with

(3) $\quad P_{y}$ playing a minor role in this entire process; and

(4) most of the vigorous reaction then occurs on wave reflection off the 2 nd HMX/Kel-F interface. This produces a substantial pressure increase and an increased rate of reaction.

The overall agreement of calculation and experiment for case II is fair.

\section{Case I. (PMMA/HMX/PMMA - $700 \mathrm{~m} / \mathrm{s}$ projectile velocity)}

For Case I, the scenario is much the same. The input pressure and particle speed are somewhat higher, however not high enough to kick in the major energy releasing reaction component of $C_{s}^{+}$. The transit time across the HMX is shorter for case I than for Case II (see Figs. 2a and 2b), giving a shorter time for reaction buildup. When the wave hits the 2nd HMX/PMMA interface the pressure increases; however PMMA provides poorer confinement than does Kel-F . As a result, the increasing rate of reaction produced by the interaction (and the corresponding pressurization) is easily reduced by the ease with which the PMMA sandwich is disassembled. That is, PMMA doesn't provide enough confinement to maintain the pressurization process. Consequently, although a substantial amount of combustion occurs, no self-confinement is possible and the detonation-like wave behavior seen in the experiment is not replicated in the calculation.

From these comparisons it is clear that the reaction rate used by Baer to model DDT does not adequately reproduce the shock initiation-like experiments that have been done. I anticipate that we could come up with a better rate law to do this part of the problem, using existing data.

The real question (concerning safety) that we need to address is not so much questions concerning the vigorous reaction seen in these experiments, but rather lower pressure, longer time combustion ignition reactions. Also the large wave reflections occurring at the 2nd HMX/inert interface need to be minimized. There is little doubt that a 10-20 kbar pressure will lead to detonation in these damaged materials. This is what the wave reflections are doing. The real regime of interest is the lower pressure, much longer time regime. Perhaps a Shepherd-test like porous inert in place of full density plastic @ the 2 nd HMX/inert interface would help. This all needs more thought. 
(Baer Model)

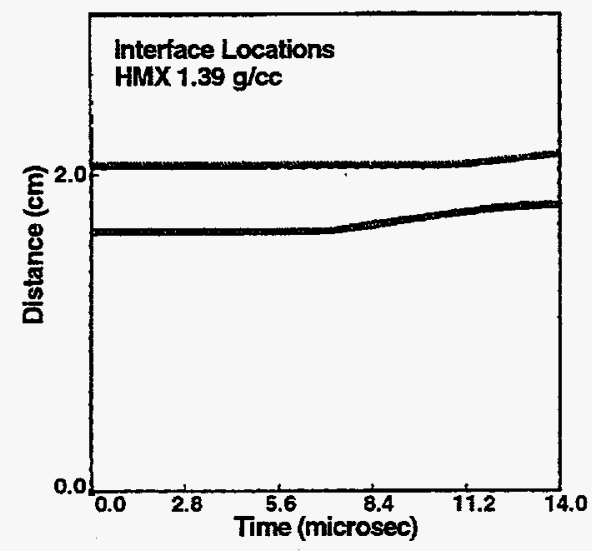

(Experiment)
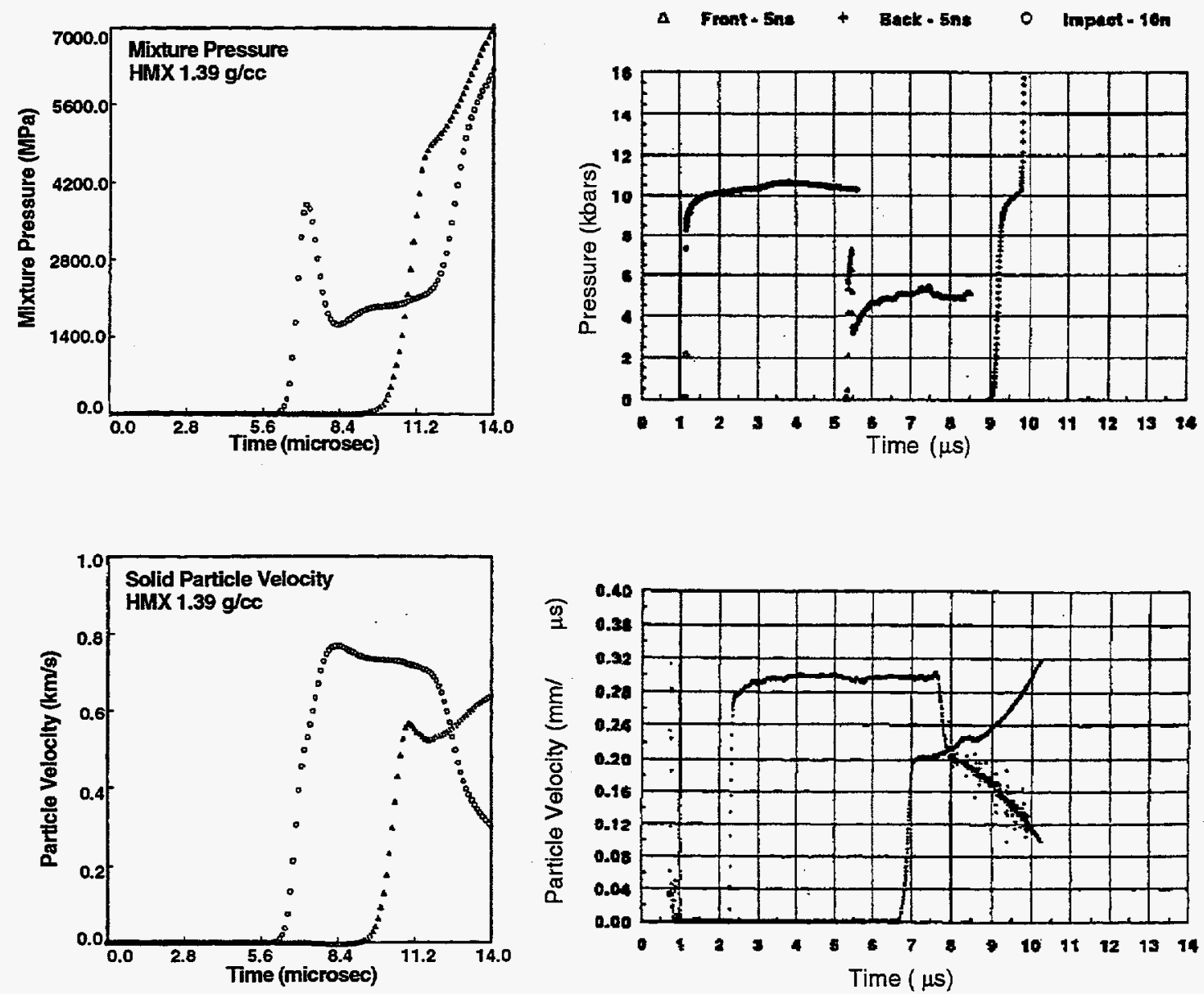

Figure 2a. Case II: Kel-F/HMX/Kel-F (4.25 mm HMX, $\left.\varphi_{s 0}=0.73\right)$ 
(Baer Model)

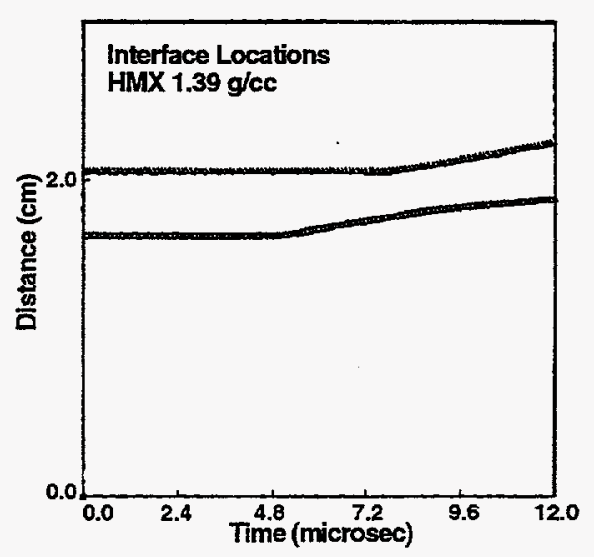

(Experiment)

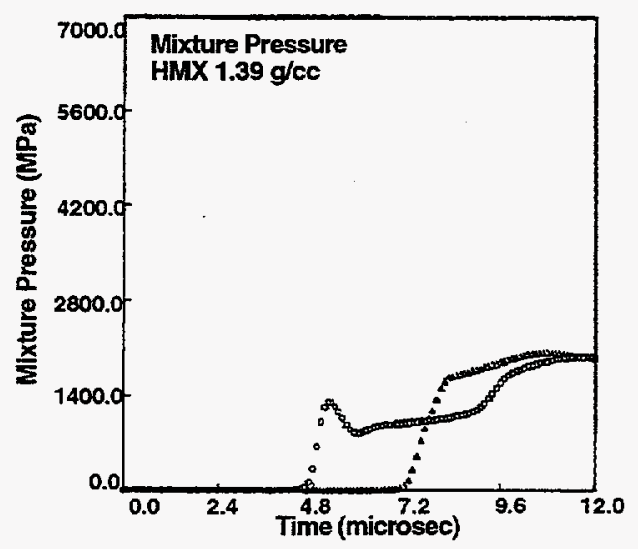

$\Delta$ Front-5ns + Back-5na 0 impact-5ns
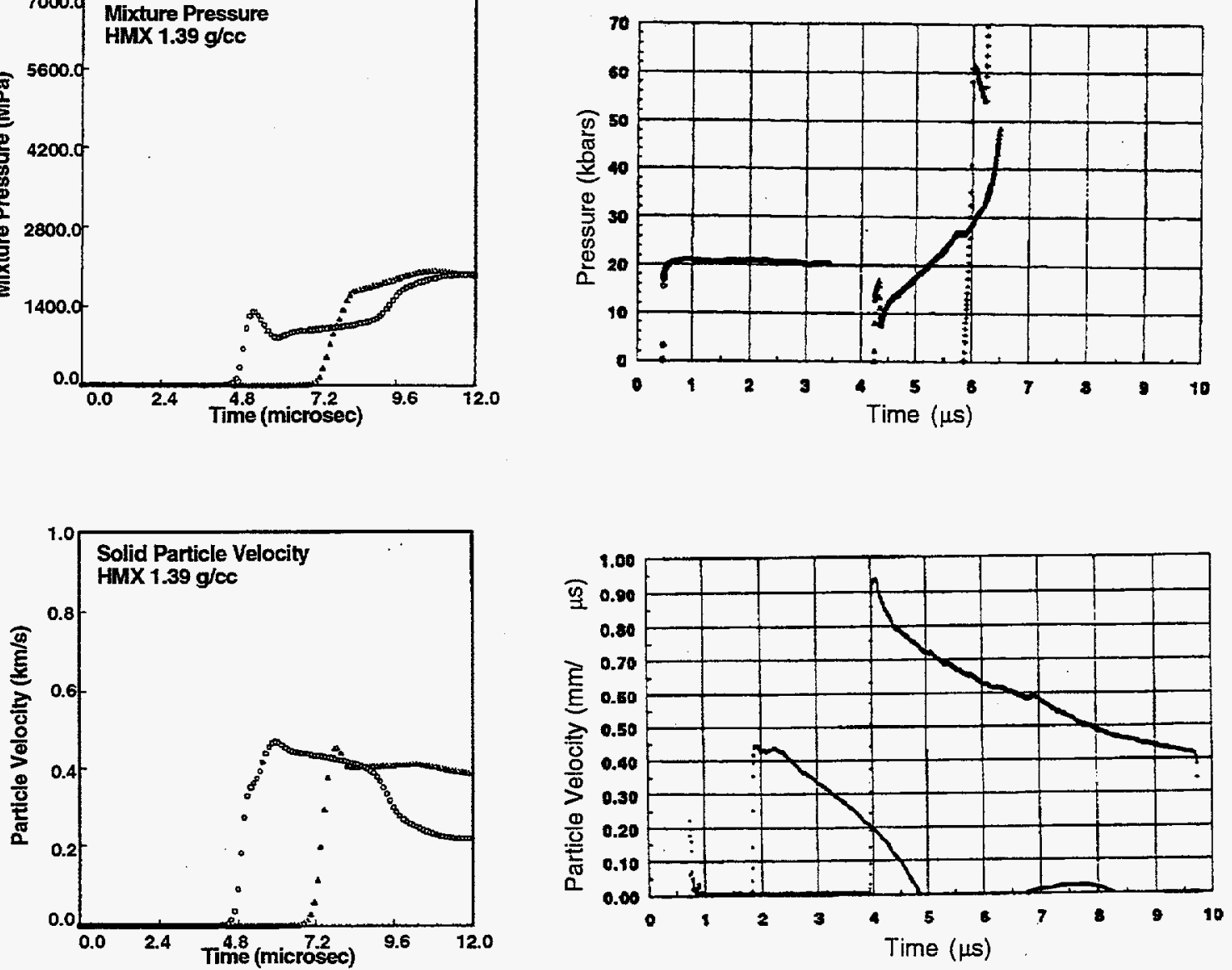

Figure 2b. Case I: PMMA/HMX/PMMA (4.25 mm HMX, $\left.\phi_{S o}=0.73\right)$ 
[1] Baer, M. R. and Nunziato, J. W., "Compressive Combustion of Granular Materials Induced by Low-Velocity Impact," Ninth symposium (International) on Detonation, Portland, OR Aug. 28-Sept. 1, 1989, pp. 293-319.

[2] Sandusky, H. W. and Bernecker, R. R. "Compressive Reaction in Porous Beds of Energetic Materials," Eighth Symposium (International) on Detonation, Albuquerque, NM, July 15-19, 1985, pp. 881-891. 
Appendix B

COMMENTS ON LARGE-DRAG REDUCTION

Los Alamos National Laboratory memo DX-16:94-99 


\section{Los Alamos}

NATIONAL LABORATORY

\section{memorandum}

\author{
EXPLOSIVES TECHNOLOGY \\ DX-16
}

TOMS: Distribution

From/MS: Steve Son, DX-16, MS C920

Phone/FAX: (505)665-0380/(505)667-0500

Symbol: DX-16:94-99

Date: April, 201994

\section{SUBJECT: COMMENTS ON THE LARGE-DRAG REDUCTION OF THE BAER-NUNZIATO (BN) MODEL}

This memo summarizes recent analytical and numerical analyses of the large drag limit of the Baer-Nunziato (BN) model (Baer and Nunziato, 1986) performed by John Bdzil, DX-10, and Steve Son. Helpful suggestions from Ash Kapila, RPI, are also acknowledged. Shocks in a two-phase material in which the only interaction is the drag between the gas and solid are considered. A two-velocity version ("2v") of the BKS code "September" was also implemented and compared with the BKS model and analysis. This study shows that the approximation used for $\delta\left(v_{s}-v_{g}\right)$ in the BKS model is incomplete at shocks. The reason for this is that it was assumed in the derivation of the BKS model that the velocity difference, $\left(v_{s}-v_{g}\right)$, was $O(1 / \delta)$ everywhere. However, the analysis shows that this is not the case. Specifically, velocity differences are generally $O(1)$ in shocks. At low gas densities and wave speeds this error is small, but at higher gas densities and wave speeds the errors become substantial in the gas phase properties.

\section{Introduction}

It is advantageous to reduce the two phase equations describing DDT phenomena to a simplified form in which the advection processes occur at a single velocity. The practical importance is that this equation structure most closely matches that already used in standard hydrocodes. Furthermore, the simplification may be less computationally intensive since it would not be necessary to resolve the thin drag relaxation zone. This limit is also justified by experimental permeation studies that report very large drag coefficients in typical granular explosives. An alternative simplification is to consider a single phase model. The SPA model (Stewart, et al., 1994) is an example of this approach. However, difficulties arise in the closure approximations necessary to formulate an effective single phase model using realistic equations of state without approximations such as pressure and temperature equilibrium. In the original SPA model these closure difficulties were avoided by assuming that the equation of state is the same for the unreacted and reacted explosive. As a result, the hydrodynamics of the two phases are the same. For most energetic materials the unreacted and reacted equations of state are different. As shown here, the velocity difference between the solid and gas phases is not everywhere zero. 


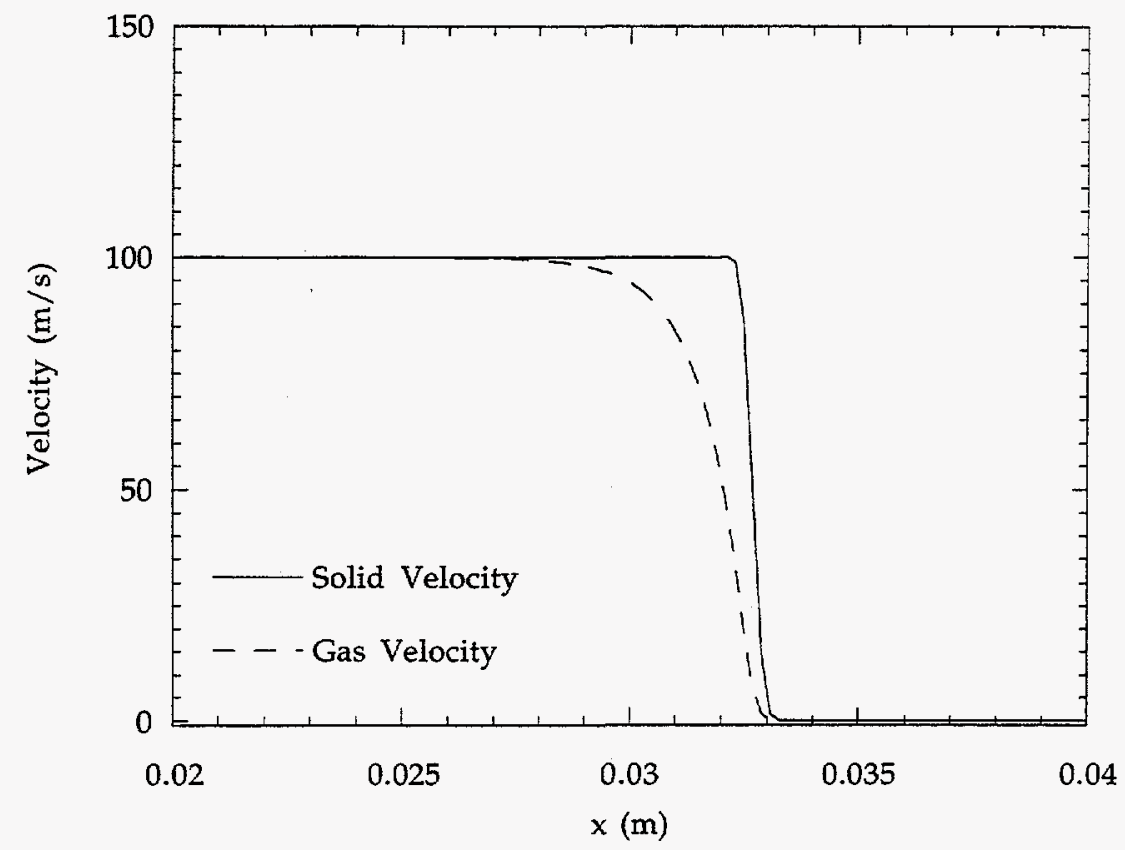

Fig. 1. Velocity profiles calculated using $2 \mathrm{v}$.

In the analysis presented here, the other rate processes such as compaction, reaction, and heat transfer interactions are not considered. As the drag coefficient becomes large, the time scale for the solid and gas velocities to relax to the same value becomes shorter than the time scales for the other rate processes mentioned above. Thus, compaction, reaction and heat transfer are frozen on the time scale of the drag interaction in the high drag limit. For supersonic compaction waves the lead disturbance is a shock, and this relaxation zone is the first part of the compaction wave structure.

In a two-phase material it is possible to have steady shocks occurring in the solid phase only, the gas phase only, or in both phases. Even in cases where both phases experience a shock simultaneously, the jumps are generally different, but then relax to the same speed in a layer whose thickness is on the order of the inverse drag coefficient, $O(1 / \delta)$. Figure 1 illustrates the typical structure of the problem considered. This result was obtained by integrating the BN (Baer and Nunziato, 1986) equations allowing only drag interaction using $2 \mathrm{v}$. In the case presented in Fig. 1, only the solid experiences a shock which results in an $O(1)$ velocity difference near the leading edge of the shock. This velocity difference then relaxes to zero through a layer of thickness $O(1 / \delta)$. As $\delta$ is increased the relaxation layer becomes thinner; however, the same structure results. Furthermore, the analysis presented below shows that the final state is independent of the form of $\delta$. The structural details presented in Fig. 1 are not observed in calculations using Baer's code to integrate the BN equations. The required numerical viscosity used in Baer's code interacts with the large default value of the drag to smear out the structure to the point where the velocities are everywhere identical. As a 
result, the final temperature in the gas is underestimated. The calculations presented in this memo were performed without added numerical viscosity. The problem considered is amenable to analysis also. This analysis is presented in the following section.

\section{Analysis}

Consider that the drag-relaxation zone is steady in a frame moving with the relaxation zone at a wave speed, $D$. Transforming the $B N$ equations to this frame and suppressing all time derivatives gives the equations for the relaxation zone structure. The two mass equations become

$$
v_{s}=D\left(1-\rho_{s}^{0} / \rho_{s}\right)
$$

and

$$
v_{g}=D\left(1-\rho_{g}^{0} / \rho_{g}\right)
$$

where $v, D$, and $\rho$ are velocity, wave speed and density, respectively. The superscript " 0 " indicates an initial undisturbed state, and the subscript "g" and "s" refer to the gas and solid state, respectively. The velocity difference is obtained by subtracting Eqs. (1) and (2),

$$
v_{s}-v_{g}=D\left(\rho_{g}^{0} / \rho_{g}-\rho_{s}^{0} / \rho_{s}\right)
$$

Since the overall momentum equation is free of sources, the jump in the overall momentum to any point in the relaxation zone is given by

$$
\phi_{s}\left(p_{s}-p_{s}^{0}\right)+\phi_{g}\left(p_{g}-p_{g}^{0}\right)=D^{2}\left\{\phi_{s} \rho_{s}^{0}\left(1-\rho_{s}^{0} / \rho_{s}\right)+\phi_{g} \rho_{g}^{0}\left(1-\rho_{g}^{0} / \rho_{g}\right)\right\},
$$

where $p$ is pressure and $\phi$ is volume fraction. We also use Eq. (4) as a mixture Rayleigh line. To that end, we rewrite Eq. (4) as

$$
\phi_{s}\left(p_{s}-p_{s}^{0}\right)+\phi_{g}\left(p_{g}-p_{g}^{0}\right)=D^{2}\left\{\left(\phi_{s} \rho_{s}^{0}+\phi_{g} \rho_{g}^{0}\right)-\frac{\left(\phi_{s} \rho_{s}^{0}\right)^{2}\left(\phi_{g} \rho_{g}\right)+\left(\phi_{g} \rho_{g}^{0}\right)^{2}\left(\phi_{s} \rho_{s}\right)}{\left(\phi_{s} \rho_{s}\right)\left(\phi_{g} \rho_{g}\right)}\right\}
$$

The overall energy equation is also in conservative form. Integrating the overall energy equation through the relaxation zone, using Eqs. (1) and (2), and rearranging terms, we get

$$
\begin{aligned}
& \phi_{s}\left(p_{s}-p_{s}^{0}\right)-\phi_{s} \rho_{s}^{0}\left\{e_{s}+p_{s} / \rho_{s}-e_{s}^{0}-p_{s}^{0} / \rho_{s}^{0}+v_{s}^{2} / 2\right\} \\
& \quad+\phi_{g}\left(p_{g}-p_{g}^{0}\right)-\phi_{g} \rho_{g}^{0}\left\{e_{g}+p_{g} / \rho_{g}-e_{g}^{0}-p_{g}^{0} / \rho_{g}^{0}+v_{g}^{2} / 2\right\}=0,
\end{aligned}
$$


where $e$ is the internal energy. In this analysis we neglect both compaction and reaction so that both $\phi_{s}$ and $\phi_{g}$ are constants; that is,

$$
\phi_{s}=\text { constant }, \quad \phi_{g}=1-\phi_{s} \text {. }
$$

In addition, the mechanical equations of state (EOS) for both the solid and gas are available,

$$
e_{s}=e_{s}\left(p_{s}, \rho_{s}\right)
$$

and

$$
e_{g}=e_{g}\left(p_{g}, \rho_{g}\right)
$$

This is a system of 6 equations and 8 unknowns. Given one more condition we can solve for 7 of the unknowns in terms of the eighth. Here we use the solid density ratio, $\rho_{s}^{0} / \rho_{s}$, as the eighth variable. An eighth equation would be required to define the spatial structure of the relaxation zone.

As our seventh equation we use the equation for the solid energy. When the $B N$ energy equation is written in terms of the internal rather than the total energy and we assume only drag interaction the state of the solid follows along an isentrope (see details below). In particular, the solid is on an isentrope after the leading shock has been traversed. Multiplying the BN solid momentum equation by $v_{s}$ and then subtracting this result from the solid energy equation gives

$$
\frac{\partial}{\partial t}\left(\phi_{s} \rho_{s} e_{s}\right)+v_{s} \frac{\partial}{\partial x}\left(\phi_{s} \rho_{s} e_{s}\right)+\left(\phi_{s} \rho_{s} e_{s}+\phi_{s} p_{s}\right) \frac{\partial v_{s}}{\partial x}=0
$$

Using the solid mass equation to eliminate $\frac{\partial \nu_{s}}{\partial x}$ and applying $\phi_{s}=$ constant yields

$$
\frac{\partial e_{s}}{\partial t}+v_{s} \frac{\partial e_{s}}{\partial x}-\frac{p_{s}}{\rho_{s}^{2}}\left\{\frac{\partial \rho_{s}}{\partial t}+v_{s} \frac{\partial \rho_{s}}{\partial x}\right\}=0
$$

or

$$
\frac{D e_{s}}{D t}-\frac{p_{s}}{\rho_{s}^{2}} \frac{D \rho_{s}}{D t}=0
$$
where here $D / D t$ is the substantial derivative, $\frac{\partial()}{\partial t}+v_{s} \frac{\partial()}{\partial x}$. By the chain rule
we have

$$
\frac{D e_{s}}{D t}=\left(\frac{\partial e_{s}}{\partial \rho_{s}}\right)_{p_{s}} \frac{D \rho_{s}}{D t}+\left(\frac{\partial e_{s}}{\partial p_{s}}\right)_{\rho_{s}} \frac{D p_{s}}{D t},
$$


which when substituted into Eq. (11), yields

$$
\left(\frac{\partial e_{s}}{\partial \rho_{s}}\right)_{p_{s}} \frac{D \rho_{s}}{D t}+\left(\frac{\partial e_{s}}{\partial p_{s}}\right)_{\rho_{s}} \frac{D p_{s}}{D t}-\frac{p_{s}}{\rho_{s}^{2}} \frac{D \rho_{s}}{D t}=0
$$

From the definition of the frozen sound speed, $c_{s}$,

$$
c_{s}^{2} \equiv\left(\frac{\partial p_{s}}{\partial \rho_{s}}\right)_{I}=\left[\frac{\frac{p_{s}}{\rho_{s}^{2}}-\left(\frac{\partial e_{s}}{\partial \rho_{s}}\right)_{p_{s}}}{\left(\frac{\partial e_{s}}{\partial p_{s}}\right)_{\rho_{s}}}\right],
$$

where the subscript " $\mathrm{I}$ " indicates an isentropic condition. It follows that Eq. (13) can be written as

$$
c_{s}^{2} \frac{D \rho_{s}}{D t}-\frac{D p_{s}}{D t}=0
$$

In a flow without discontinuities this equation describes an isentropic flow. Given the solid phase EOS, $c_{s}^{2}$ can be obtained and Eq. (15) can be integrated to obtain the isentropic relationship between solid pressure and density, which is the seventh equation used in this analysis. For simple EOS expressions, $c_{s}^{2}$ is separable and an algebraic expression can be obtained from Eq. (15). If the gas energy equation were chosen instead of the solid energy (Eq. 10) results in,

$$
c_{g}^{2} \frac{D \rho_{g}}{D t}-\frac{D p_{g}}{D t}=-\frac{\delta\left(v_{s}-v_{g}\right)^{2}}{\left(\partial e_{g} / \partial p_{g}\right)_{\rho_{g}}} .
$$

The source term in Eq. (16) produces an anisentropic flow. This illustrates a fundamental asymmetry between the gas and solid in the $\mathrm{BN}$ equations.

The EOS for solid and gas phases must now be specified. The Tait EOS was chosen for the solid, and a polytropic EOS was assumed for the gas phase. Specifically, we assumed the following:

$$
e_{s}=\frac{\left(p_{s}-p_{s}^{0}\right) / \rho_{s}}{\gamma_{s}-1}-\frac{\gamma_{s} a_{\text {Tait }}}{\left(\gamma_{s}-1\right) \rho_{s}}\left(\frac{\rho_{s}}{\rho_{s}^{0}}-1\right)
$$

where

and

$$
a_{\text {Tait }}=\left(\frac{\gamma_{s}-1}{\gamma_{s}}\right) \rho_{s}^{0}\left(\gamma_{s}-1\right) C_{v s} T_{s}^{0}
$$




$$
e_{g}=\frac{p_{g} / \rho_{g}}{\gamma_{g}-1}
$$

where $C_{v s^{\prime}}\left(\gamma_{s}-1\right)$, and $\gamma_{g}$ are the solid phase specific heat, solid Gruneisen parameter, and gas polytropic exponent, respectively.

These forms were chosen because the Hayes and JWL (equations of state currently used in September and $2 \mathrm{v}$ ) simplify to the Tait and ideal gas expressions by appropriate choice of EOS constants so direct comparisons can easily be made between analysis and numerical results. The sound speeds using these EOS expression are

$$
\begin{gathered}
c_{s}^{2}=\gamma_{s}\left(p_{s}-p_{s}^{0}+a_{\text {Tait }}\right) / \rho_{s}+\left(\gamma_{s}-1\right) \frac{p_{s}^{0}}{\rho_{s}}, \\
c_{g}^{2}=\frac{\gamma_{g} p_{g}}{\rho_{g}},
\end{gathered}
$$

and

$$
c_{\text {mix }}=\sqrt{\frac{\rho_{s} \phi_{s} c_{s}^{2}+\rho_{g} \phi_{g} c_{g}^{2}}{\rho_{s} \phi_{s}+\rho_{g} \phi_{g}}}
$$

where $c_{\text {mix }}$ is the mixture sound speed. Calculating the sound speeds is useful in eliminating some solutions as unphysical, as well as, obtaining the seventh equation from Eq. (15). For example, a wave for which the flow in both phases is acoustically disconnected from the piston is unphysical. To ensure that the piston is acoustically connected to the wavefront, $v+c-D>0$, for at least one of the phases.

Since the solid flow is isentropic (except through jumps) a relationship exists between solid pressure and density. Using Eq. (17), and Eq. (14), the solid isentrope is found to be

$$
p_{s}=\frac{p_{s}^{0}}{\gamma_{s}}-a_{\text {Tait }}+\left(\frac{\rho_{s}^{0}}{\rho_{s}^{+}}\right)^{\gamma_{s}}\left(\frac{\rho_{s}}{\rho_{s}^{0}}\right)^{\gamma_{s}}\left(p_{s}^{+}+a_{\text {Tait }}-p_{s}^{0} / \gamma_{s}\right),
$$

where a shock is assumed in the solid. The " + " indicates the shock value. This reduces to

$$
p_{s}=\frac{p_{s}^{0}}{\gamma_{s}}-a_{\text {Tait }}+\left(\frac{\rho_{s}}{\rho_{s}^{0}}\right)^{\gamma_{s}}\left(p_{s}^{0}+a_{\text {Tait }}-p_{s}^{0} / \gamma_{s}\right)
$$


when a shock does not occur in the solid. The solid phase Hugoniot can now be obtained using the solid phase energy jump condition,

$$
\left(p_{s}^{+}-p_{s}^{0}\right)\left\{\frac{1}{\rho_{s}^{0}}-\left(\frac{\gamma_{s}+1}{\gamma_{s}-1}\right)\left(\frac{1}{\rho_{s}^{+}}\right)\right\}+2\left\{p_{s}^{0}+\frac{\gamma_{s} a_{\text {Tait }}}{\gamma_{s}-1}\right\}\left(\frac{1}{\rho_{s}^{0}}-\frac{1}{\rho_{s}^{+}}\right)=0 \text {. }
$$

Using the solid phase momentum and mass jump conditions, and Eq. (23), the following expressions are obtained:

$$
\begin{gathered}
\frac{\rho_{s}^{0}}{\rho_{s}^{+}}=\left(\frac{\gamma_{s}-1}{\gamma_{s}+1}\right)\left\{1+\frac{2}{\rho_{s}^{0} D^{2}}\left(p_{s}^{0}+\frac{\gamma_{s} a_{\text {Tait }}}{\gamma_{s}-1}\right)\right\} \\
p_{s}^{+}=\frac{2 \rho_{s}^{0} D^{2}}{\gamma_{s}-1}-\left(\frac{2 \gamma_{s}}{\gamma_{s}+1}\right) a_{\text {Tait }}+p_{s}^{0}\left(\frac{\left(3-\gamma_{s}\right)}{\left(\gamma_{s}+1\right)}\right) .
\end{gathered}
$$

The remaining task is to solve the system of algebraic equations. First, the equations are combined and simplified. Using the assumed EOS forms for the gas and solid, the overall energy equation, Eq. (6), becomes,

$$
\begin{gathered}
\phi_{s} \frac{\gamma_{s}}{\gamma_{s}-1}\left(p_{s}-p_{s}^{0}\right) \frac{\rho_{s}^{0}}{\rho_{s}}-\phi_{s}\left(p_{s}^{0}+\frac{\gamma_{s} a_{\text {Tait }}}{\gamma_{s}-1}\right)\left(1-\frac{\rho_{s}^{0}}{\rho_{s}}\right) \\
+\phi_{g} \frac{\gamma_{g}}{\gamma_{g}-1}\left(p_{g}-p_{g}^{0}\right) \frac{\rho_{g}^{0}}{\rho_{g}}-\phi_{g} \frac{\gamma_{g}}{\gamma_{g}-1} p_{g}^{0}\left(1-\frac{\rho_{g}^{0}}{\rho_{g}}\right) \\
=\frac{1}{2} \phi_{s} \rho_{s}^{0} D^{2}\left(1-\left(\frac{\rho_{s}^{0}}{\rho_{s}}\right)^{2}\right)+\frac{1}{2} \phi_{g} \rho_{g}^{0} D^{2}\left(1-\left(\frac{\rho_{g}^{0}}{\rho_{g}}\right)^{2}\right) .
\end{gathered}
$$

Now, $p_{g}-p_{g}^{0}$ can be eliminated using the overall momentum equation, Eq. (4). After some manipulation an equation for the density ratio difference is obtained that has the following quadratic form:

$$
A\left(\rho_{g}^{0} / \rho_{g}-\rho_{s}^{0} / \rho_{s}\right)^{2}+B\left(\rho_{g}^{0} / \rho_{g}-\rho_{s}^{0} / \rho_{s}\right)+C=0
$$

where

$$
\begin{gathered}
A \equiv \phi_{g} \rho_{g}^{0} \frac{D^{2}}{2}\left(\frac{\gamma_{g}+1}{\gamma_{g}-1}\right) \\
B \equiv-\frac{\gamma_{g}}{\gamma_{g}-1}\left\{\left(\phi_{g} \rho_{g}^{0}+\phi_{s} \rho_{s}^{0}\right) D^{2}+\phi_{g} p_{g}^{0}+\phi_{s} p_{s}^{0}-\phi_{s} p_{s}-\left(\phi_{s} \rho_{s}^{0}+\phi_{g} \rho_{g}^{0} \frac{\gamma_{g}+1}{\gamma_{g}}\right) D^{2} \frac{\rho_{s}^{0}}{\rho_{s}}\right\}
\end{gathered}
$$

and 


$$
\begin{aligned}
C & \equiv \frac{1}{2}\left(\frac{\gamma_{g}+1}{\gamma_{g}-1}\right)\left(\phi_{g} \rho_{g}^{0}+\phi_{s} \rho_{s}^{0}\right) D^{2}\left(\frac{\rho_{s}^{0}}{\rho_{s}}\right)^{2}+\left(\frac{\gamma_{g}}{\gamma_{g}-1}-\frac{\gamma_{s}}{\gamma_{s}-1}\right) \phi_{s}\left(p_{s}-p_{s}^{0}\right) \frac{\rho_{s}^{0}}{\rho_{s}} \\
& -\frac{\gamma_{g}}{\gamma_{g}-1}\left\{\left(\phi_{g} \rho_{g}^{0}+\phi_{s} \rho_{s}^{0}\right) D^{2}+\phi_{g} p_{g}^{0}+\phi_{s} \frac{\gamma_{g}-1}{\gamma_{g}}\left(p_{s}^{0}+\frac{\gamma_{s} a_{\text {Tait }}}{\gamma_{s}-1}\right)\right\} \frac{\rho_{s}^{0}}{\rho_{s}} \\
& +\frac{1}{2}\left(\phi_{g} \rho_{g}^{0}+\phi_{s} \rho_{s}^{0}\right) D^{2}+\frac{\gamma_{g}}{\gamma_{g}-1} \phi_{g} p_{g}^{0}+\phi_{s}\left(p_{s}^{0}+\frac{\gamma_{s} a_{\text {Tait }}}{\gamma_{s}-1}\right) .
\end{aligned}
$$

An interesting observation is that the form of the drag does not enter into either the final equilibrium state or the relaxation structure.

Equation (28) can now be evaluated. The first step in the evaluation procedure is to determine the state $\left(\rho_{s}^{0} / \rho_{s}\right)$ at the end of the viscous relaxation layer where the velocities are equal and consequently, $\rho_{g}^{0} / \rho_{g}-\rho_{s}^{0} / \rho_{s}=0$. Equation (28) then simplifies to $C=0$. Equation (22) is used when a solid shock is assumed to occur and Eq. (23) is used when a solid shock is assumed not to occur. Both possibilities are considered. The quadratic equation, Eq. (28), is then solved and evaluated for the density ratio difference, $\rho_{g}^{0} / \rho_{g}-\rho_{s}^{0} / \rho_{s}$, as a function of $\rho_{s}^{0} / \rho_{s}$ through the relaxation layer.

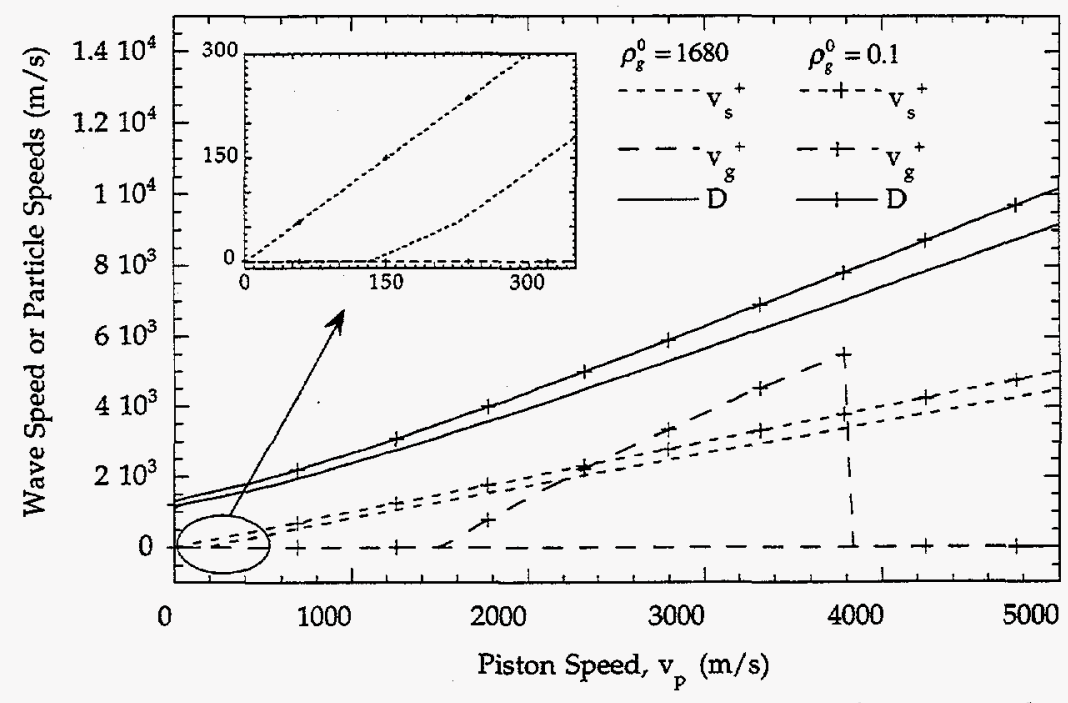

Fig. 2a. Plot of wave speed, $D$, and particle speeds as a function of piston speed using the parameters: $\phi_{s}=0.73, \gamma_{s}=3, \rho_{s}^{0}=1900 \mathrm{~kg} / \mathrm{m}^{3}$, $p_{s}^{0}=10^{6} \mathrm{~Pa}, c_{v s}=1500 \mathrm{~J} / \mathrm{kg} / \mathrm{K}, \gamma_{\mathrm{g}}=1.25, p_{g}^{0}=10^{6} \mathrm{~Pa}$, $c_{v g}=2375.64 \mathrm{~J} / \mathrm{kg} / \mathrm{K}$. 


\section{Evaluation and Comparison with Numerics}

Figures $2 a-2 c$ illustrate typical results obtained from the analysis. Figure $2 a$ is a plot of the wave speed and particle velocities as functions of piston speed. Figures $2 b$ and $2 c$ are plots of the sound speeds and acoustic parameters as functions of the piston speed, respectively. Two gas density values are shown. The high gas density example examines the case of nearly equal solid and gas densities. This corresponds to conditions that may occur in problems that involve reaction and secondary compaction waves. At wave speeds below the solid sound speed in the undisturbed state, no shocks occur for the cases considered (see Figs. $2 \mathrm{a}$ and $2 \mathrm{~b}$ ). That is, the solid particle speed, $u_{s}^{+}$, and the gas particle speed, $u_{g}^{+}$, are zero at low piston speeds, $u_{p}$ (see inset on Fig. $2 a)$. For $D$ above the solid phase sound speed $(1342 \mathrm{~m} / \mathrm{s}$ for the cases considered), a solid phase shock occurs $\left(u_{s}^{+}>0\right)$. At high wave speeds both solutions assuming a solid shock wave and no solid shock wave are possible solutions. In the Figs. $2 a-2 c$, the solutions obtained assuming a solid shock are used for $D$ above the solid sound speed $(1342 \mathrm{~m} / \mathrm{s})$ and the solutions obtained assuming no solid shock are used for $D$ below the solid sound speed. Numerical calculations using $2 \mathrm{v}$ match the solution assuming a shock in the solid for $D$ above the solid sound speed. Furthermore, a higher piston speed is required to attain the same wave speed for the case that assumes no solid shock. Perhaps a minimum entropy criterion could be used to select the stable solution. A solution with a gas shock but no solid shock has not been observed for $\rho_{s}^{0}>\rho_{g}^{0}$. At very high porosities, one observes a gas shock and no solid shock.

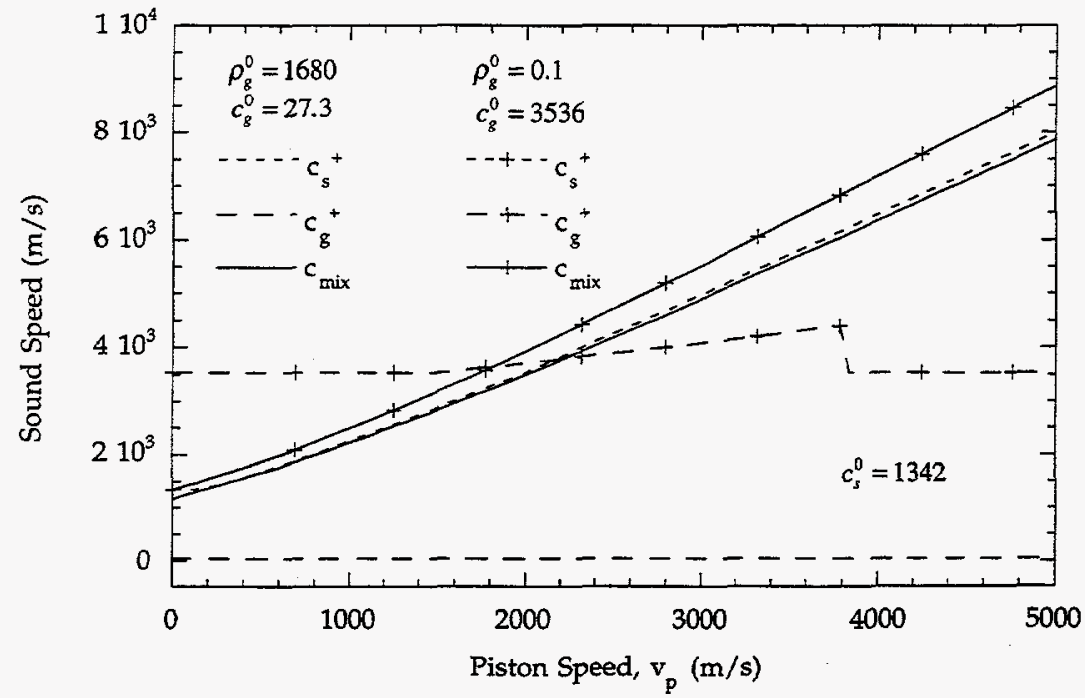

Fig. 2b. Plot of sound speeds as a function of piston speed using the parameters: $\phi_{s}=0.73, \gamma_{s}=3, \rho_{s}^{0}=1900 \mathrm{~kg} / \mathrm{m}^{3}, p_{s}^{0}=10^{6} \mathrm{~Pa}$, $c_{v s}=1500 \mathrm{~J} / \mathrm{kg} / \mathrm{K}, \gamma_{g}=1.25, p_{g}^{0}=10^{6} \mathrm{~Pa}, c_{v g}=2375.64 \mathrm{~J} / \mathrm{kg} / \mathrm{K}$. 


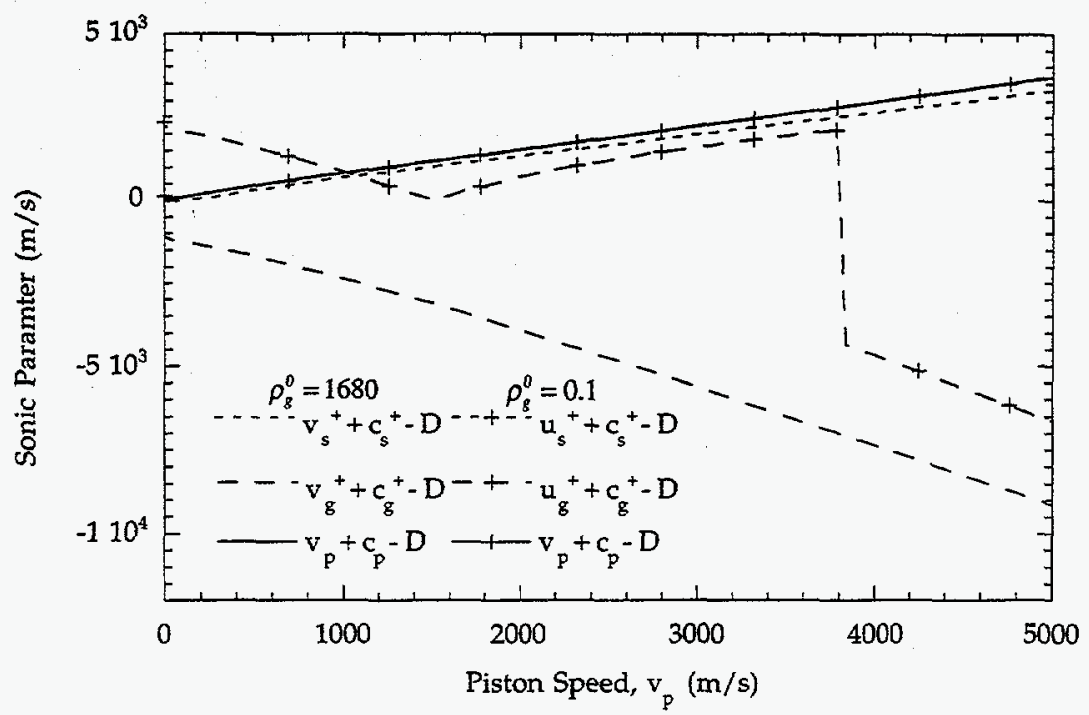

Fig. 2c. Plot of the sonic parameter as a function of piston speed using the parameters: $\phi_{s}=0.73, \gamma_{s}=3, \rho_{s}^{0}=1900 \mathrm{~kg} / \mathrm{m}^{3}, p_{s}^{0}=10^{6} \mathrm{~Pa}$, $c_{v s}=1500 \mathrm{~J} / \mathrm{kg} / \mathrm{K}, \gamma_{g}=1.25, p_{g}^{0}=10^{6} \mathrm{~Pa}, c_{v g}=2375.64 \mathrm{~J} / \mathrm{kg} / \mathrm{K}$.

In the high gas density case the mixture sound speed is somewhat lower than the solid sound speed (see Fig. 2b). Consequently, a weak wave forms at low piston speeds that propagates near the mixture sound speed (see plot of $D$ in Fig. 2a). As the piston speed increases, the mixture sound speed increases and the wave speed of the weak wave increases. (see Figs. $2 a$ and $2 b$ ). For the low gas density case shown, a weak wave (no shocks) propagating at the mixture sound speed evaluated at the piston is only possible for a narrow range of piston speeds and the solid shock appears at very low piston speeds (see inset on Fig. 2a). In the low gas density case, a gas and solid shock can occur simultaneously over a range of wave speeds. The gas shock appears when the wave speed, $D$, reaches the undisturbed gas sound speed and it disappears where it becomes acoustically disconnected (see Fig. 2c) from the piston at high wave speeds. For the high density case the gas phase sonic parameter is always negative which indicates it is always acoustically disconnected from the piston (see Fig. 2c). For the low gas density case it is acoustically connected at low piston speeds, but becomes disconnected at higher piston speeds. The point where it becomes disconnected corresponds to piston speed where the gas shock no longer occurs (compare Figs. 2a and 2c). These results show that energy from the solid typically flows from the solid phase to the gas phase througth the drag interaction. This is especially true when the gas phase is acoustically disconnected and only a solid shock occurs.

Another view of the solution is obtained by considering the Hugoniot plots. Figure 3 shows the mixture Hugoniot plot. The ordinate comes from 
consideration of Eq. (5) and was chosen to yield a linear Rayleigh line for the mixture. The abscissa uses the mixture pressure, $\langle p\rangle$,

$$
\langle p\rangle \equiv \phi_{s} p_{s}+\phi_{g} p_{g}
$$

normalized by the initial mixture pressure. A single state path corresponding to a wave speed, $D$, of $6500 \mathrm{~m} / \mathrm{s}$ is also shown. The mixture experiences a shock in the solid phase that takes it to the shock state. At this point, the gas phase particle velocity is zero and the solid particle velocity is at its shocked value. Therefore, the velocity difference is order one. The mixture density and pressure continue to increase through the relaxation zone until the velocities have equilibrated. For comparison the solution was evaluated assuming a zero gas density $\left(\rho_{g}^{0}=0\right)$. This results in the "P- $\alpha$ " approximation (Caroll and Holt, 1971). Significant differences are observed between the zero gas density Hugoniot and the final Hugoniot that results from this analysis. Of course, for low gas densities the agreement is better. However, with reaction and compaction present, high gas densities occur in problems of interest in DDT.

The solid and gas phase Hugoniots for this case are also shown (Figs. 4 \& 5). As discussed above, the solid flow is isentropic through the relaxation region. The gas phase does not experience a shock under these conditions but does experience an anisentropic compression due to the drag interaction. For reference, the gas isentrope is also plotted in Fig. 5. The anisentropic process greatly influences the final state achieved, as shown in Fig. 5.

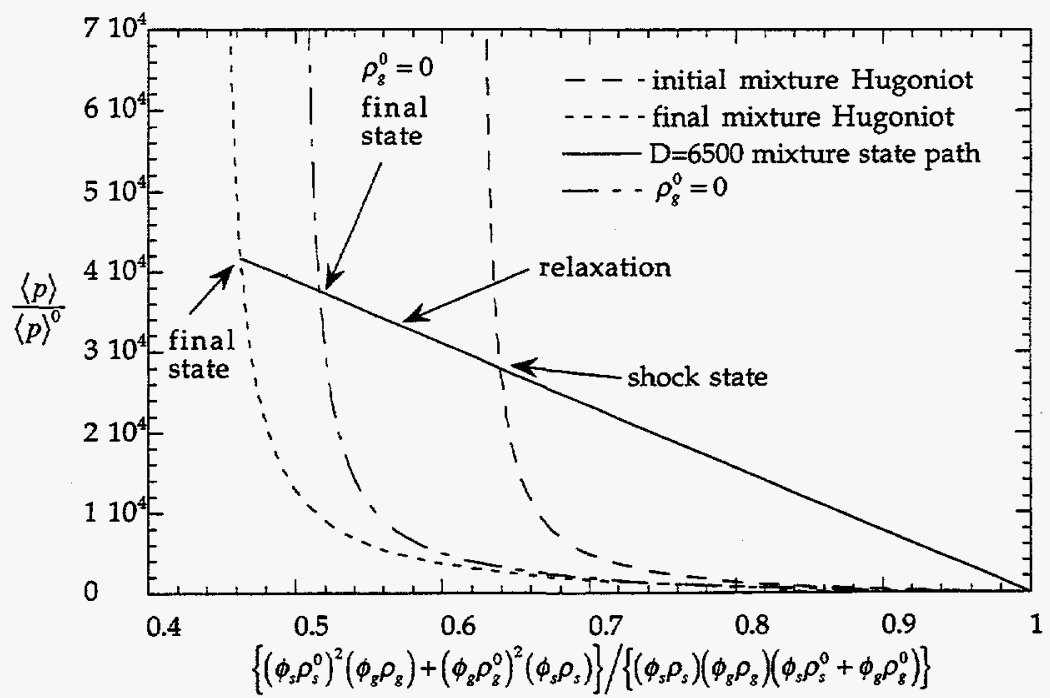

Fig. 3. Mixture Hugoniot plot using the parameters: $\phi_{s}=0.73, \gamma_{s}=3$, $\rho_{s}^{0}=1900 \mathrm{~kg} / \mathrm{m}^{3}, p_{s}^{0}=10^{6} \mathrm{~Pa}, c_{v s}=1500 \mathrm{~J} / \mathrm{kg} / \mathrm{K}, \gamma_{g}=1.25$, $\rho_{g}^{0}=1680 \mathrm{~kg} / \mathrm{m}^{3}$ (except $\rho_{g}^{0}=0$ case), $p_{s}^{0}=10^{6} \mathrm{~Pa}, c_{v g}=2375.64 \mathrm{~J} / \mathrm{kg} / \mathrm{K}$. 


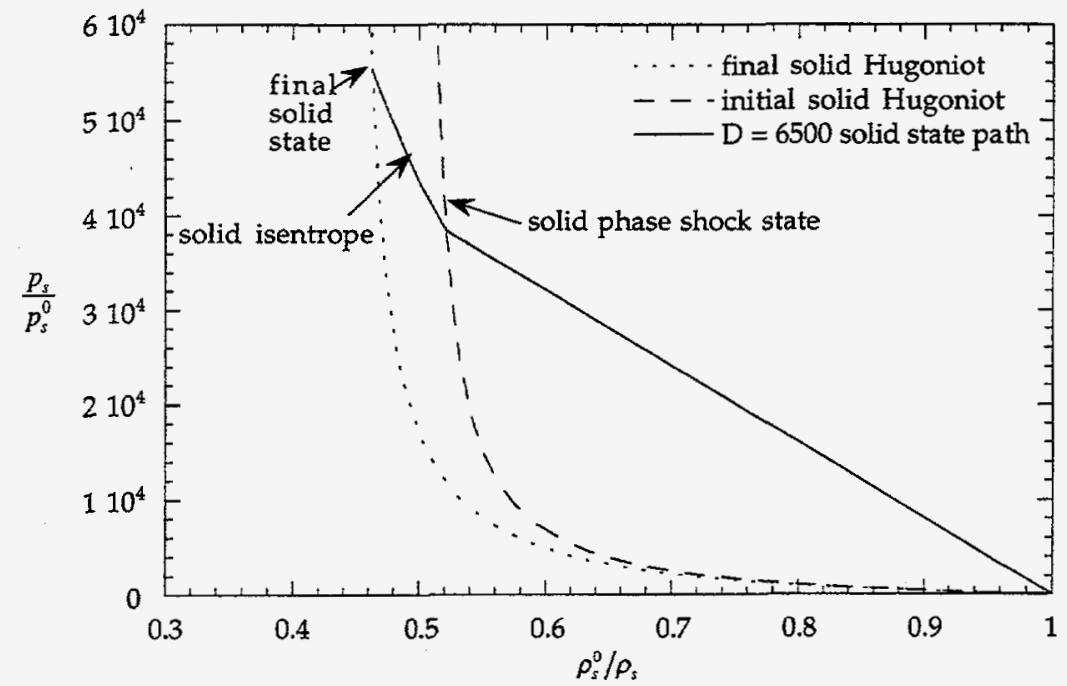

Fig. 4. Solid phase Hugoniot plot using the parameters: $\phi_{s}=0.73, \gamma_{s}=3$, $\rho_{s}^{0}=1900 \mathrm{~kg} / \mathrm{m}^{3}, p_{s}^{0}=10^{6} \mathrm{~Pa}, c_{v s}=1500 \mathrm{~J} / \mathrm{kg} / \mathrm{K}, \gamma_{g}=1.25$, $\rho_{g}^{0}=1680 \mathrm{~kg} / \mathrm{m}^{3}, p_{g}^{0}=10^{6} \mathrm{~Pa}, c_{v g}=2375.64 \mathrm{~J} / \mathrm{kg} / \mathrm{K}$.

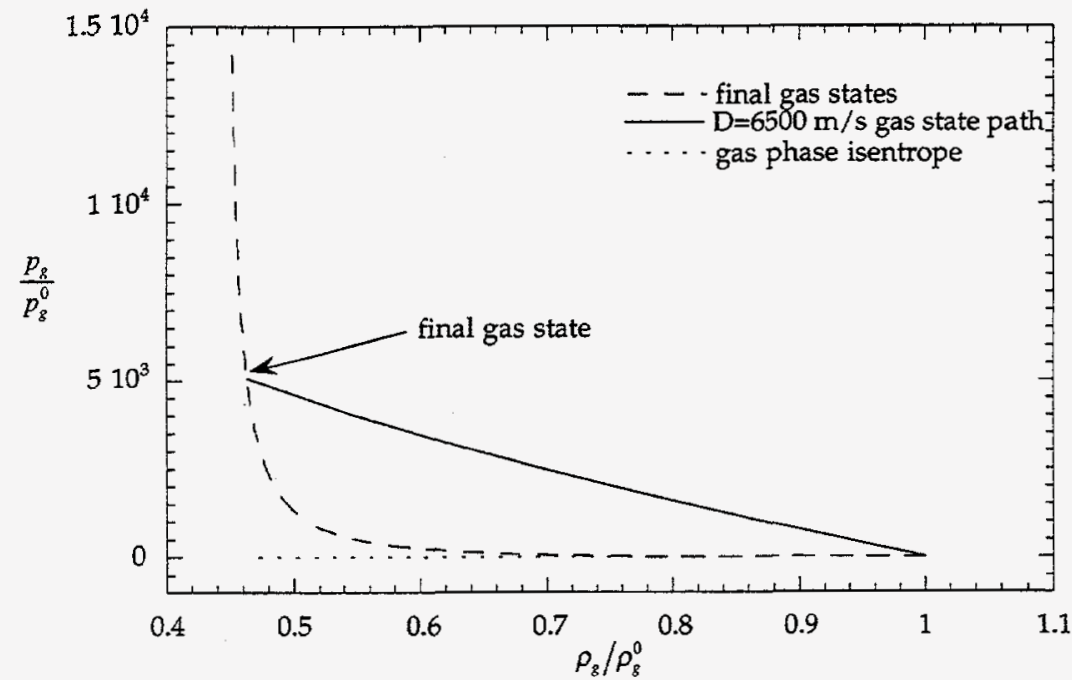

Fig. 5. Gas phase Hugoniot plot using the parameters: $\phi_{s}=0.73, \gamma_{s}=3$, $\rho_{s}^{0}=1900 \mathrm{~kg} / \mathrm{m}^{3}, p_{s}^{0}=10^{6} \mathrm{~Pa}, c_{v s}=1500 \mathrm{~J} / \mathrm{kg} / \mathrm{K}, \gamma_{g}=1.25$ ， $\rho_{g}^{0}=1680 \mathrm{~kg} / \mathrm{m}^{3}, p_{g}^{0}=10^{6} \mathrm{~Pa}, c_{v g}=2375.64 \mathrm{~J} / \mathrm{kg} / \mathrm{K}$. 


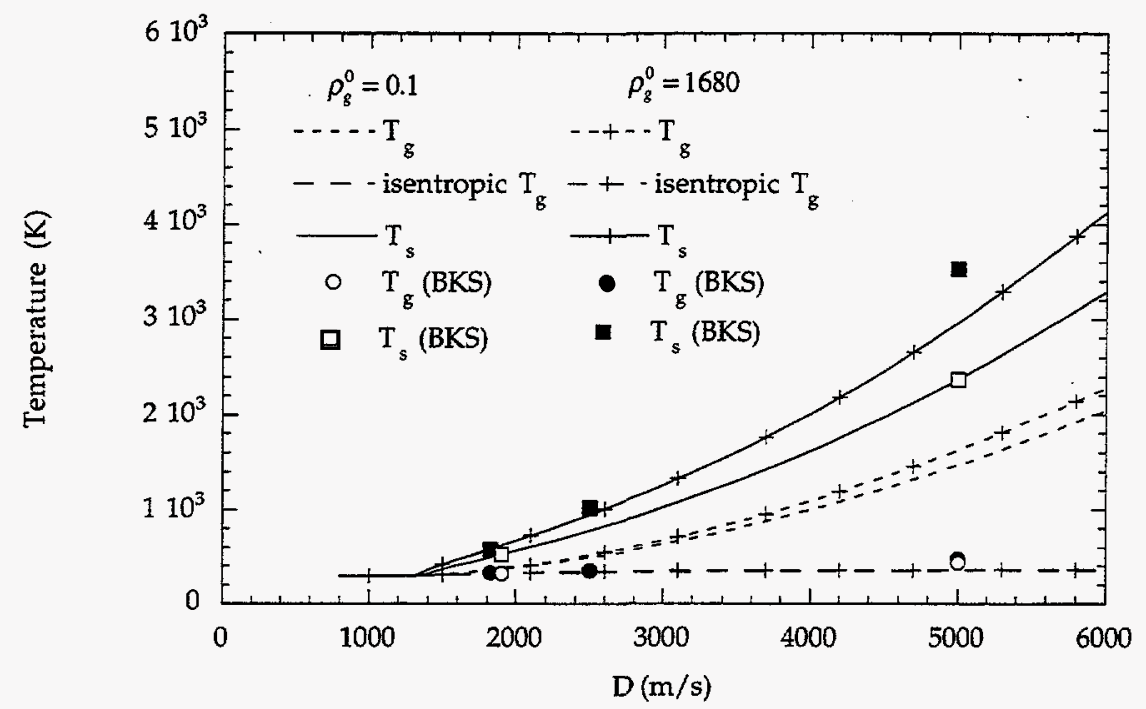

Fig. 6. Temperatures using the parameters: $\phi_{s}=0.73, \gamma_{s}=3, \rho_{s}^{0}=1900 \mathrm{~kg} / \mathrm{m}^{3}$, $T_{s}^{0}=300 \mathrm{~K}, c_{v s}=1500 \mathrm{~J} / \mathrm{kg} / \mathrm{K}, \gamma_{g}=1.25, T_{g}^{0}=300 \mathrm{~K}, c_{v g}=2375.64 \mathrm{~J} / \mathrm{kg} / \mathrm{K}$.

The gas and solid temperatures as a function of wave speed are shown in Fig. 6. In these calculations the gas and solid temperatures were set to $300 \mathrm{~K}$ and the initial pressure was varied. The accurate partition of internal energy between the solid and gas phase in a mixture experiencing a shock and drag interaction is likely important. This is especially true if reaction takes place after this process. The partition of energy will also affect compaction. A gas shock is not present under these conditions, but the gas is heated by the drag interaction with the solid. The gas temperature achieved if the gas flow were isentropic is shown for comparison. These results show that the gas temperature is affected by the anisentropic process. The temperatures are somewhat higher for the high gas density case. This is likely due to the higher drag experienced on the solid by the greater gas mass. Also, a few calculations using the BKS model are shown. At low wave speeds and low gas densities the comparison is fair. However, at higher wave speeds, the deviation becomes significant in even the low gas density case. The transfer of energy from the solid to the gas is under predicted by the BKS model and consequently the temperature is higher in the solid than it should be and lower in the gas. Interestingly, the temperature of the solid is greater than the temperature of the gas. Because of its lower wave speed, the gas plays the role of an energy sink in the relaxation zone.

Table 1 summarizes several comparisons made between the analysis, the BKS (September) model and the BN (2v) model. These calculations are fairly well resolved, but grid independence was not assured in every case; however, the calculations are accurate enough for our purposes. Generally, at low wave speeds and gas densities the comparison is good for all variables. However, at 
higher wave speeds and gas densities the errors by BKS (September), particularly in gas pressure, become significant. The simulations using the $\mathrm{BN}(2 \mathrm{v})$ model compare well with the analysis for all cases considered.

We have recently formulated a more general large-drag reduced model. This model requires that the relaxation zone be resolved using an equation for the velocity difference. The advantage of this is that the advection is still performed at a single velocity which makes implementation into standard hydrocodes relatively straightforward. Initial calculations adding the additional equation appear promising; however, the necessity of resolving the relaxation layer has numerical penalties such as those observed with $2 \mathrm{v}$. Since the BKS model performs reasonably well at lower wave speeds and gas densities, and the solid phase is calculated reasonably well by BKS at all wave speeds, it may be adequate to use BKS for all cases. Furthermore, significant differences between BN (using $2 \mathrm{v}$ ) and BKS (September) on problems with additional interactions such as compaction and reaction have not been observed for several cases considered. This indicates that the errors in the gas phase made by BKS in the shock may not affect the final state or the other processes significantly. 
Table 1. Comparison of Analysis with Numerical Models*

Input

Parameters

\begin{tabular}{|c|c|c|c|c|}
\hline (1) $\rho_{g}^{0}=0.1$ & $\mathrm{D}$ & 4600 & 4569 & 4591 \\
\hline$u_{p}=2104$ & $\rho_{s}^{0} / \rho_{s}$ & 0.5426 & 0.5424 & 0.5424 \\
\hline$p_{g}^{0}=p_{s}^{0}=10^{6}$ & $p_{s}$ & $1.8392 \times 10^{10}$ & $1.839 \times 10^{10}$ & $1.839 \times 10^{10}$ \\
\hline$\phi_{s}=0.73$ & $p_{g}$ & $2.1559 \times 10^{6}$ & $2.179 \times 10^{6}$ & $2.170 \times 10^{6}$ \\
\hline (2) $\rho_{g}^{0}=0.1$ & D & 5905 & 5922 & 5900 \\
\hline$u_{p}=2800$ & $\rho_{s}^{0} / \rho_{s}$ & 0.52582 & 0.5258 & 0.5256 \\
\hline$p_{g}^{0}=p_{s}^{0}=10^{6}$ & $p_{s}$ & $3.1416 \times 10^{10}$ & $3.1417 \times 10^{10}$ & $3.1417 \times 10^{10}$ \\
\hline$\phi_{s}=0.2$ & $p_{g}$ & $2.3136 \times 10^{6}$ & $2.2831 \times 10^{6}$ & $2.2654 \times 10^{6}$ \\
\hline (3) $\rho_{g}^{0}=0.1$ & $\bar{D}$ & 6000 & 5997 & 6009 \\
\hline$u_{p}=2850$ & $\rho_{s}^{0} / \rho_{s}$ & 0.5201 & 0.5248 & 0.5250 \\
\hline$p_{g}^{0}=p_{s}^{0}=10^{6}$ & $p_{s}$ & $3.2490 \times 10^{10}$ & $3.2492 \times 10^{10}$ & $3.2490 \times 10^{10}$ \\
\hline$\phi_{s}=0.73$ & $p_{g}$ & $2.3243 \times 10^{6}$ & $2.2936 \times 10^{6}$ & $2.2697 \times 10^{6}$ \\
\hline (4) $\rho_{g}^{0}=0.1$ & $\bar{D}$ & 9000 & 9009 & 8662 \\
\hline$u_{p}=4130$ & $\rho_{s}^{0} / \rho_{s}$ & 0.51112 & 0.51119 & 0.50905 \\
\hline$p_{g}^{0}=p_{s}^{0}=10^{6}$ & $p_{s}$ & $7.5241 \times 10^{10}$ & $7.5243 \times 10^{10}$ & $6.6470 \times 10^{10}$ \\
\hline$\phi_{s}=0.73$ & $p_{g}$ & $2.6691 \times 10^{6}$ & $2.4956 \times 10^{6}$ & $2.3572 \times 10^{6}$ \\
\hline (5) $\rho_{g}^{0}=0.1$ & D & 1934 & 1940 & 1934 \\
\hline$u_{p}=500$ & $\rho_{s}^{0} / \rho_{s}$ & 0.74144 & 0.74117 & 0.74132 \\
\hline$p_{g}^{0}=p_{s}^{0}=10^{6}$ & $p_{s}$ & $1.8350 \times 10^{9}$ & $1.8336 \times 10^{9}$ & $1.8340 \times 10^{9}$ \\
\hline$\phi_{s}=0.1$ & $p_{g}$ & $1.4401 \times 10^{6}$ & $1.4680 \times 10^{6}$ & $1.4555 \times 10^{6}$ \\
\hline (6) $\rho_{g}^{0}=0.1$ & $\bar{D}$ & 1900 & 1899 & 1910 \\
\hline$u_{p}=476.3$ & $\rho_{s}^{0} / \rho_{s}$ & 0.74930 & 0.74908 & 0.74932 \\
\hline$p_{g}^{0}=p_{s}^{0}=17817$ & $p_{s}$ & $1.7196 \times 10^{9}$ & $1.7194 \times 10^{9}$ & $1.7190 \times 10^{9}$ \\
\hline$\phi_{s}=0.73$ & $p_{g}$ & 29053 & 26584 & 25733 \\
\hline (7) $\rho_{g}^{0}=0.1$ & $\mathrm{D}$ & 5000 & & 5005 \\
\hline$u_{p}=2320$ & $\rho_{s}^{0} / \rho_{s}$ & 0.53600 & & 0.53594 \\
\hline$p_{g}^{0}=p_{s}^{0}=17817$ & $p_{s}$ & $2.2041 \times 10^{10}$ & & $2.2040 \times 10^{10}$ \\
\hline$\phi_{s}=0.73$ & $p_{g}$ & $1.6262 \times 10^{5}$ & & $4.8003 \times 10^{4}$ \\
\hline (8) $\rho_{g}^{0}=5.6$ & $\overline{\mathrm{D}}$ & 1900 & 1908 & 1901 \\
\hline$u_{p}=477$ & $\rho_{s}^{0} / \rho_{s}$ & 0.74910 & 0.74892 & 0.74892 \\
\hline$p_{g}^{0}=p_{s}^{0}=10^{6}$ & $p_{s}$ & $1.7236 \times 10^{9}$ & $1.7240 \times 10^{9}$ & $1.7234 \times 10^{9}$ \\
\hline$\phi_{s}=0.73$ & $p_{g}$ & $1.63127 \times 10^{6}$ & $1.6231 \times 10^{6}$ & $1.4392 \times 10^{6}$ \\
\hline
\end{tabular}




\begin{tabular}{c|lccc}
\hline (9) $\rho_{g}^{0}=5.6$ & $\mathrm{D}$ & 4224 & 4232 & 4220 \\
$u_{p}=1900$ & $\rho_{s}^{0} / \rho_{s}$ & 0.550203 & 0.55026 & 0.550397 \\
$p_{g}^{0}=p_{s}^{0}=10^{6}$ & $p_{s}$ & $1.52656 \times 10^{10}$ & $1.52656 \times 10^{10}$ & $1.5270 \times 10^{10}$ \\
$\phi_{s}=0.73$ & $p_{g}$ & $6.6230 \times 10^{6}$ & $6.5608 \times 10^{6}$ & $2.4370 \times 10^{6}$ \\
\hline $\mathbf{( 1 0 )} \rho_{g}^{0}=5.6$ & $\mathrm{D}$ & 1880 & 1890 & 1896 \\
$u_{p}=476.7$ & $\rho_{s}^{0} / \rho_{s}$ & 0.74636 & 0.74662 & 0.74714 \\
$p_{g}^{0}=p_{s}^{0}=10^{6}$ & $p_{s}$ & $1.7429 \times 10^{9}$ & $1.7435 \times 10^{9}$ & $1.7500 \times 10^{9}$ \\
$\phi_{s}=0.1$ & $p_{g}$ & $1.6322 \times 10^{6}$ & $1.6306 \times 10^{6}$ & $1.4495 \times 10^{6}$ \\
\hline $\mathbf{( 1 1 )} \rho_{g}^{0}=1680$ & $\mathrm{D}$ & 1713 & 1732 & 1769 \\
$u_{p}=499.8$ & $\rho_{s}^{0} / \rho_{s}$ & 0.70822 & 0.7086 & 0.7090 \\
$p_{g}^{0}=p_{s}^{0}=10^{6}$ & $p_{s}$ & $2.13947 \times 10^{9}$ & $2.1440 \times 10^{9}$ & $2.222 \times 10^{9}$ \\
$\phi_{s}=0.73$ & $p_{g}$ & $5.5830 \times 10^{7}$ & $5.3565 \times 10^{7}$ & $5.2663 \times 10^{6}$ \\
\hline $\mathbf{( 1 2 )} \rho_{g}^{0}=1680$ & $\mathrm{D}$ & 500.1 & 515.4 & 505.5 \\
$u_{p}=50$ & $\rho_{s}^{0} / \rho_{s}$ & 0.90002 & 0.90056 & 0.899621 \\
$p_{g}^{0}=p_{s}^{0}=10^{6}$ & $p_{s}$ & $4.2495 \times 10^{8}$ & $4.2245 \times 10^{8}$ & $4.2660 \times 10^{8}$ \\
$\phi_{s}=0.1$ & $p_{g}$ & $1.1873 \times 10^{6}$ & $1.2069 \times 10^{6}$ & $1.1569 \times 10^{6}$ \\
\hline $\mathbf{( 1 3 )} \rho_{g}^{0}=1680$ & $\mathrm{D}$ & 1800 & 1823 & 1842 \\
$u_{p}=480.65$ & $\rho_{s}^{0} / \rho_{s}$ & 0.73297 & 0.73359 & 0.73895 \\
$p_{g}^{0}=p_{s}^{0}=3 \times 10^{8}$ & $p_{s}$ & $2.4125 \times 10^{9}$ & $2.4205 \times 10^{9}$ & $2.4820 \times 10^{9}$ \\
$\phi_{s}=0.73$ & $p_{g}$ & $4.8619 \times 10^{8}$ & $4.8145 \times 10^{8}$ & $4.4124 \times 10^{8}$ \\
\hline $\mathbf{( 1 4 )} \rho_{g}^{0}=1680$ & $\mathrm{D}$ & 2500 & & 2625 \\
$u_{p}=1014.3$ & $\rho_{s}^{0} / \rho_{s}$ & 0.59430 & & 0.61426 \\
$p_{g}^{0}=p_{s}^{0}=3 \times 10^{8}$ & $p_{s}$ & $6.4829 \times 10^{9}$ & & $6.9260 \times 10^{9}$ \\
$\phi_{s}=0.73$ & $p_{g}$ & $8.6890 \times 10^{8}$ & & $5.7705 \times 10^{8}$ \\
\hline $\mathbf{( 1 5 )} \rho_{g}^{0}=1680$ & $\mathrm{D}$ & 5000 & & 5450 \\
$u_{p}=2589.4$ & $\rho_{s}^{0} / \rho_{s}$ & 0.48211 & & 3.523396 \\
$p_{g}^{0}=p_{s}^{0}=3 \times 10^{8}$ & $p_{s}$ & $3.1812 \times 10^{10}$ & & $8.8465 \times 10^{8}$ \\
\hline$\phi_{s}=0.73$ & $p_{g}$ & $3.3641 \times 10^{9}$ & & \\
\hline
\end{tabular}

* all mks units. Other input parameters used were, unless otherwise specified: $\phi_{s}=0.73, \gamma_{s}=3, c_{v s}=1500 \mathrm{~J} / \mathrm{kg} / \mathrm{K}, c_{v g}=2375.64 \mathrm{~J} / \mathrm{kg} / \mathrm{K}$.

\section{References}

Baer, M. R. and J. W. Nunziato (1986), "A Two-Phase Mixture Theory for the Deflagration-to-Detonation Transition (DDT) in Reactive Granular Materials," International Journal of Multiphase Flow, Vol. 12, No. 6, pp. 86189. 
Stewart, D. S., Asay, B. W, and Prasad, K. (1994), Physics of Fluids, "Simplified Modeling of Transition to Detonation in Porous Energetic Materials".

Distribution:

Blaine Asay, DX-16, MS C920

John Bdzil, DX-10, MS P952

Jay Dallman, DX-16,MS C920

Bill Davis, DX-10, MS P952

Larry Hill, DX-10, MS P952

Phil Howe, DX-DO, MS P915

Deanne Idar, DX-16, MS C920

Ash Kapila, kapila@rpi.edu

Jon Mace, DX-16, MS C920

John McAfee, DX-10, MS P950

Ralph Menikoff, T-14, MS B214

Scott Stewart, ds-stewart@uiuc.edu

DX-16 File 